\title{
MEASUREMENT AND MODELING OF BLOCKING CONTACTS FOR CADMIUM TELLURIDE GAMMA RAY DETECTORS
}

\author{
A Thesis presented to the \\ Electrical Engineering Faculty of \\ California Polytechnic State University, San Luis Obispo \\ In Partial Fulfillment \\ of the Requirements for the Degree \\ Master of Science in Electrical Engineering
}

by

Patrick R. Beck

December 2009 
(C) 2009

Patrick R. Beck

ALL RIGHTS RESERVED 


\section{COMMITTEE MEMBERSHIP}

TITLE:

AUTHOR:

DATE SUBMITTED:

COMMITTEE CHAIR: William Ahlgren

COMMITTEE MEMBER: Dennis Derickson

Dec 11, 2009

\author{
Measurement and Modeling of Blocking Contacts for \\ Cadmium Telluride Gamma Ray Detectors \\ Patrick R. Beck
}

COMMITTEE MEMBER: Xiaomin Jin 


\author{
ABSTRACT \\ Measurement and Modeling of Blocking Contacts for \\ Cadmium Telluride Gamma Ray Detectors \\ Patrick R. Beck
}

Gamma ray detectors are important in national security applications, medicine, and astronomy. Semiconductor materials with high density and atomic number, such as Cadmium Telluride (CdTe), offer a small device footprint, but their performance is limited by noise at room temperature; however, improved device design can decrease detector noise by reducing leakage current.

This thesis characterizes and models two unique Schottky devices: one with an argon ion sputter etch before Schottky contact deposition and one without. Analysis of current versus voltage characteristics shows that thermionic emission alone does not describe these devices. This analysis points to reverse bias generation current or leakage through an inhomogeneous barrier. Modeling the devices in reverse bias with thermionic field emission and a leaky Schottky barrier yields good agreement with measurements. Also numerical modeling with a finite-element physics-based simulator suggests that reverse bias current is a combination of thermionic emission and generation.

This thesis proposes further experiments to determine the correct model for reverse bias conduction. Understanding conduction mechanisms in these devices will help develop more reproducible contacts, reduce leakage current, and ultimately improve detector performance. 


\section{ACKNOWLEGEMENTS}

I want to thank Dr. Ahlgren and Dr. Adam Conway for your guidance and all the

time put into reviewing my work. Without Dr. Ahlgren's direction, this thesis would have grown out of control. I want to also thank Dr. Jin and Dr. Derickson for taking the time to serve on my thesis committee.

Although I performed all measurement, analysis, and modeling described in this thesis, the CdZnTe research team was responsible for the physical device design and fabrication. Thank you to the CdZnTe team for your feedback and support: Adam Conway, Steve Payne, Tim Graff, Lars Voss, Rebecca Nikolic, Art Nelson, Tzu Fang Wang, Elaine Behymer, and Ben Sturm. Thank you also to Bruce Henderer, Lawrence Livermore National Laboratory, and Cal Poly for giving me this opportunity. This work was performed under the auspices of the U.S. DOE by Lawrence Livermore National Laboratory under contract DE-AC52-07NA27344, LLNL-TH-425864. 


\section{TABLE OF CONTENTS}

LIST OF TABLES

VIII

LIST OF FIGURES

IX

1. INTRODUCTION

\section{1}

1.1 Design, Measurement, ANd MOdeling Summary 2

1.2 DOCUMENT OVERVIEW 2

2. BACKGROUND 3

2.1 GAMMA RAY DETECTORS 3

2.2 High Resistivity Cadmium TelluRide

3. INITIAL DESIGN 12

3.1 Silvaco Device Simulator

3.2 DETECTOR DESIGN

3.3 Physical DeVice Design

4. DEVICE FABRICATION AND MEASUREMENT 23

4.1 FABRicATION $\quad 24$

4.2 MeASUREMENTS

4.2.1 KeITHLEY 4200 SEMICONDUCTOR CHARACTERIZATION SYSTEM 28

4.2.2 DEVICE MEASUREMENTS 28

4.2.3 MEASUREMENT ERROR SOURCES 29

5. MEASUREMENT RESULTS AND ANALYSIS 32

5.1 THERMIONIC EMISSION

5.1.1 CurRent Versus Voltage EXTRACTION METHOD 35

5.1.2 CurRent VERSuS TEMPERATURE EXTRACTION METHOD 39

5.1.3 EXTRACTION RESULTS 41

5.2 LEAKY SCHOTTKY BARRIER MODEL 48 
6.1 SIMULATION EXPERIMENTS

6.1.1 TRAP DENSITY $\quad 56$

6.1.1.1 LOW TRAP DENSITY

6.1.1.2 HIGH TRAP DENSITY 62

6.1.1.3 CARRIER LIFETIME 64

6.1.2 CONTACT AREA 66

6.1.3 SIMULATION EXPERIMENT SUMMARY $\quad 67$

6.2 COMPARISON With DEVICE MEASUREMENTS

6.3 RESOLVING DIFFERENCES IN NUMERICAL AND ANALYTICAL MODELS 72

\begin{tabular}{lr} 
7. CONCLUSIONS AND FUTURE WORK & 77 \\
\hline
\end{tabular}

7.1 ANalytical ANd NuMERICAL MOdeling 77

$\begin{array}{ll}7.2 \text { FUTURE WORK } & 79\end{array}$

\begin{tabular}{lr} 
REFERENCES & 81 \\
\hline
\end{tabular}

APPENDIX A: SILVACO ATLAS SIMULATION CODE $\quad 88$ 


\section{LIST OF TABLES}

Table 3.1 User-Defined Material Parameters for CdTe at 300K

Table 3.2 Mobility Temperature Dependence Model

Table 4.1 Device Geometry

Table 4.2 Keithley 4200 SCS Measurement Accuracy

Table 5.1 Extracted Values for $500 \mu \mathrm{m}$ Diameter No Sputter Etch Device

Table 5.2 Extracted $500 \mu \mathrm{m}$ Device Parameters at Room Temperature

Table 5.3 Extracted $250 \mu \mathrm{m}$ Device Parameters at Room Temperature

Table 5.4 Leaky Schottky Barrier Model Parameters for $500 \mu \mathrm{m}$ Devices

Table 5.5 Defect Levels Accounting for Barrier Leakage Resistance

Table 6.1 Simulation Matrix

Table 6.2 Summary of Device Parameter Effects

Table 6.3 Numerical Model Parameters for $500 \mu \mathrm{m}$ No Sputter Etch Device

Table 6.4 Voltage and Temperature Dependence of Possible Current Mechanisms

Table 7.1 Extracted Barrier Heights for Analytical and Numerical Models 


\section{LIST OF FIGURES}

Figure 2.1 Energy Spectrums for Gamma Detectors Using a Low Enriched Uranium Source [4] 4

Figure 2.2 CdTe Absorption Coefficient [6] 6

Figure 2.3 Typical Gamma Ray Structure Indicating Gamma-Generated Carriers 6

Figure 2.4 Charge Collection in $1 \mathrm{~cm}$ Thick CdTe Detector; $\mu_{\mathrm{e}} \tau_{\mathrm{e}} \approx 1 \mathrm{~ms}, \mu_{\mathrm{h}} \tau_{\mathrm{h}} \approx 0.1 \mathrm{~ms} \quad 8$

Figure 2.5 Energy Resolution Dependence on Leakage Current for a 200 keV Gamma Ray [4] 9

Figure 2.6 CdTe Zincblende Structure [12] 10

Figure 2.7 Wafer Surface Along (111) Plane [11] 11

Figure 3.1 Ohmic and Schottky Detector I-V Characteristics 13

$\begin{array}{ll}\text { Figure 3.2 Schottky Detector Concept } & 13\end{array}$

Figure 3.3 Electron and Hole Mobility Versus Temperature [21] 16

$\begin{array}{ll}\text { Figure 3.4 Simulated Detector Structure } & 17\end{array}$

Figure 3.5 Simulated Ohmic Detector Energy Band Diagram 17

$\begin{array}{ll}\text { Figure 3.6 Simulated Ohmic Detector I-V Characteristic } & 18\end{array}$

$\begin{array}{ll}\text { Figure 3.7 Schottky Barrier Height Definition } & 18\end{array}$

Figure 3.8 Schottky Detector Reverse Bias Electron Injection 19

Figure 3.9 Simulated Schottky Detector Energy Band Diagrams 20

Figure 3.10 Schottky and Ohmic Detector Current-Voltage Characteristics 21

Figure 3.11 Schottky Detector Reverse Bias Conduction 22

Figure 4.1 Argon Ion Sputter Etch; Courtesy: L. F. Voss 23

Figure 4.2 Anode Contact Geometry (Cathode Not to Scale) 25

Figure 4.3 Anode and Guard Ring Currents at Room Temperature for a Typical Device 26 
Figure 4.4 Current-Voltage Measurement System

Figure 4.5 Device I-V Measurement Configuration

Figure 4.6 Measurement Error Caused by Hot Chuck at $70^{\circ} \mathrm{C}$

Figure 5.1 Thermionic Emission Processes

Figure 5.2 Typical I-V at Room Temperature for the No Sputter Etch Device

Figure 5.3 Typical I-V for the No Sputter Etch Device

Figure 5.4 Device Structure and Equivalent Circuit

Figure 5.5 I-V Curve at Room Temperature With and Without Correcting For Series Resistance

Figure 5.6 I-V Parameter Extraction Plot Corrected For $\mathrm{R}_{\mathrm{S}}$ and Metal-Semiconductor Non-Ideality

Figure 5.7 Arrhenius Plot for Current-Temperature Parameter Extraction

Figure 5.8 Measured Low Voltage Current-Voltage Characteristic for $500 \mu \mathrm{m}$ Devices

Figure 5.9 Inhomogeneous Schottky Barrier

Figure 5.10 Ideality Factor Temperature Dependence

Figure 5.11 Measured High Voltage Current-Voltage Characteristic for $500 \mu \mathrm{m}$ Devices

Figure 5.12 Differential Resistance for $500 \mu \mathrm{m}$ Devices

Figure 5.13 Device Structure and Equivalent Circuit with Shunt Resistance

Figure 5.14 Leaky Schottky Barrier Model for $500 \mu \mathrm{m}$ Diameter No Sputter Etch Device

Figure 5.15 Leaky Schottky Barrier Model for $500 \mu \mathrm{m}$ Diameter Sputter Etch Device

Figure 5.16 Leaky Schottky Barrier Resistance for $500 \mu \mathrm{m}$ Devices

Figure 6.1 Trap Energy Level Definitions

Figure 6.2 Simulated Device Structure

Figure 6.3 Depletion Region Widths at Zero Bias 
Figure 6.4 I-V Characteristics for Device without Traps with Varying Anode Work Function

Figure 6.5 I-V Characteristics for Low Trap Density Device with Varying Anode Work Function

Figure 6.6 Low Trap Density Energy Band Diagram at Reverse Bias (+2V)

Figure 6.7 Low Trap Density Energy Band Diagram at Forward Bias (-2V)

Figure 6.8 I-V Characteristics at High Trap Density with Varying Anode Work Function

Figure 6.9 High Trap Density Energy Band Diagram in Reverse Bias (+2V)

Figure 6.10 High Trap Density Energy Band Diagram in Forward Bias (-2V)

Figure 6.11 I-V Characteristics with Varying Carrier Lifetime

Figure 6.12 I-V Characteristics with Varying Anode Size

Figure 6.13 Bandgap Temperature Dependence

Figure 6.14 High Voltage I-V Characteristic for No Sputter Etch Device

Figure 6.15 High Voltage I-V Characteristic for Sputter Etch Device

Figure 6.16 Voltage Dependence at Low Reverse Bias 


\section{INTRODUCTION}

Gamma ray detectors are important in national security applications, medicine, and astronomy; in national security, border security forces and first-responders require mobile devices that operate at room temperature. Semiconductor materials with high density and atomic number, such as Cadmium Telluride (CdTe), offer the small device footprint required for mobile applications; however, noise in these semiconductor detectors limits room temperature performance. Most research effort is in improved crystal growth and readout electronics to improve detector performance; however, improved device design can also decrease detector noise by reducing leakage current.

This thesis seeks to design detectors with reduced leakage current by replacing a typical ohmic contact with a blocking Schottky contact. While Schottky contacts are not a new technique for reducing leakage current in semiconductor radiation detectors, they are poorly understood and have issues with reproducibility. Two unique Schottky devices are fabricated with different surface treatments; characterization and modeling of these devices provides insight into current mechanisms and the effects of the surface treatments. These studies suggest ways to improve contact fabrication, reduce leakage current, and ultimately improve detector performance. 


\subsection{Design, Measurement, and Modeling Summary}

A finite-element physics-based simulator, Silvaco's Atlas, was used to perform the initial design of the contact structure. After detectors were fabricated, electrical characterization was performed using current-versus-voltage measurements. Device parameters such as Schottky barrier height, ideality factor, and reverse bias leakage current were determined from these measurements using analytical models. Additional numerical modeling was performed to include more complex physics for which an analytical solution cannot be determined.

\subsection{Document Overview}

This thesis follows the development and analysis of a Schottky gamma ray detector. Chapter two provides a basic explanation of how gamma ray detectors function and describes the semiconductor material. Chapters three and four describe device design, fabrication, and measurement. Chapters five and six describe analytical and numerical modeling, respectively. Chapter seven summarizes the results of these models and discusses future work already in progress. 


\section{BACKGROUND}

\subsection{Gamma Ray Detectors}

Gamma ray detectors operate by one of two mechanisms: scintillation or direct conversion. Scintillation detectors use photodiodes or photomultiplier tubes to detect light produced by the interaction of a gamma ray with a scintillating crystal [1]. Direct conversion detectors utilize semiconducting material to detect electrons and holes generated when the device absorbs a gamma ray. Scintillators typically have lower energy resolution for their size than direct conversion detectors.

For radioactive isotope identification, energy resolution defines detector performance. In the detected energy spectrum, the energy resolution is the ratio of a peak's energy to the full width at half max (FWHM) energy of that peak, as shown in equation $2.1[2]$, [3].

$$
\text { Energy Resolution, } R=\frac{F W H M}{E_{\gamma}}
$$

A specific combination of peaks in this spectrum defines the unique energy signature of a radioactive isotope. In order to obtain unambiguous signature identification, detectors need an energy resolution of approximately $1 \%$ [4]. Figure 2.1 shows the detected energy signature from a low enriched uranium (LEU) source for three different detectors. 


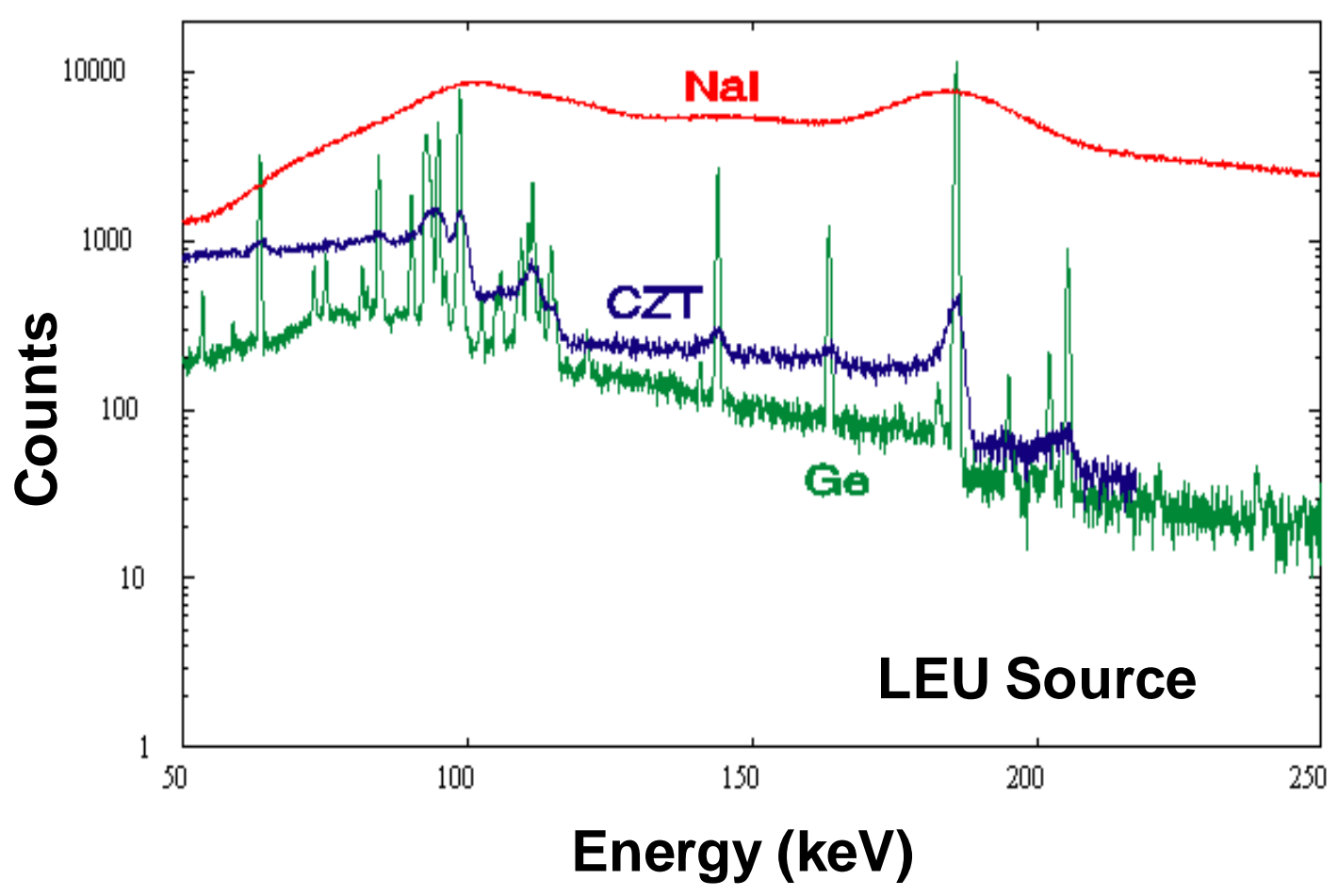

Figure 2.1 Energy Spectrums for Gamma Detectors Using a Low Enriched Uranium Source [4]

The Sodium Iodide (NaI) scintillator achieves only 6\% resolution showing almost no signature, a typical Cadmium Zinc Telluride (CZT) direct detector achieves relatively good signature detection at $2 \%$ resolution, and the Germanium $(\mathrm{Ge})$ direct detector shows a well-defined energy signature with $0.2 \%$ resolution. However, Ge is a low bandgap material that must be operated at cryogenic temperatures to reduce leakage from thermally generated carriers; the cooling requirements of Ge make it cumbersome and expensive, and thus a poor choice for mobile detectors. Detectors based on higher bandgap materials like CdTe and CZT can operate at room temperature, since they have less thermally generated carriers [4]. There are three main factors in determining detector performance: gamma ray absorption, charge collection, and detector noise. 
Semiconductor detectors absorb ionizing radiation through three mechanisms: the photoelectric effect, Compton scattering, and pair production. However, pair production only occurs when gamma ray energy exceeds twice the electron rest-mass (1.02 MeV), and this lies outside of the range of interest (about $50 \mathrm{keV}$ to $1 \mathrm{MeV}$ ). Photoelectric effect occurs predominantly at lower gamma ray energies; the atom completely absorbs a gamma ray and emits a photoelectron from a bound state, where the energy of the photoelectron is equal to the gamma ray energy minus the atomic binding energy. Photoelectric effect probability increases as $Z^{4}$ to $Z^{5}$ depending on the material, so the high atomic number of $\mathrm{Cd}(\mathrm{Z}=48)$ and $\mathrm{Te}(\mathrm{Z}=52)$ make this absorption method likely in CdTe. Absorption by Compton scattering becomes more likely at high gamma energy, where absorption via the photoelectric effect declines; in Compton scattering the gamma ray collides with an electron, imparts some energy, and scatters off in a different direction. An absorption coefficient, $\mu$, derived from these mechanisms describes the exponential decay in transmitted gamma rays:

$$
\frac{I}{I_{0}}=e^{-\mu L}
$$

where $\mathrm{I}$ is the transmitted gamma intensity, $\mathrm{I}_{0}$ is the incident gamma intensity, and $\mathrm{L}$ is the detector thickness. For example, a $1 \mathrm{~cm}$ thick CdTe detector only absorbs $36 \%$ of the incident $662 \mathrm{keV}$ gamma rays (from $\mathrm{Cs}^{137}$, a common benchmark) [3], [5]. Figure 2.2 shows the absorption coefficient for CdTe due to the three absorption methods. 


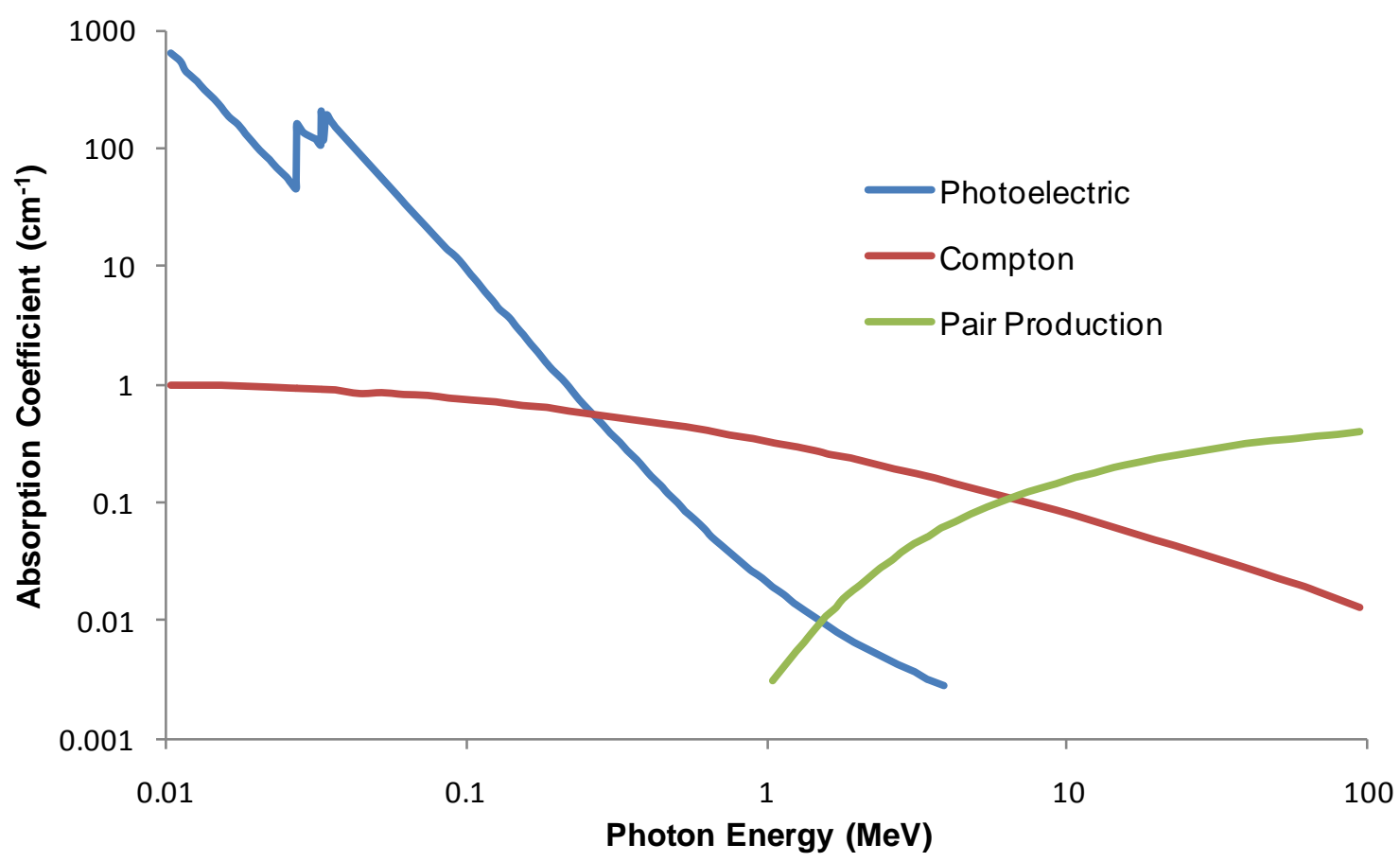

Figure 2.2 CdTe Absorption Coefficient [6]

Figure 2.3 shows the physical structure of a typical gamma ray detector.

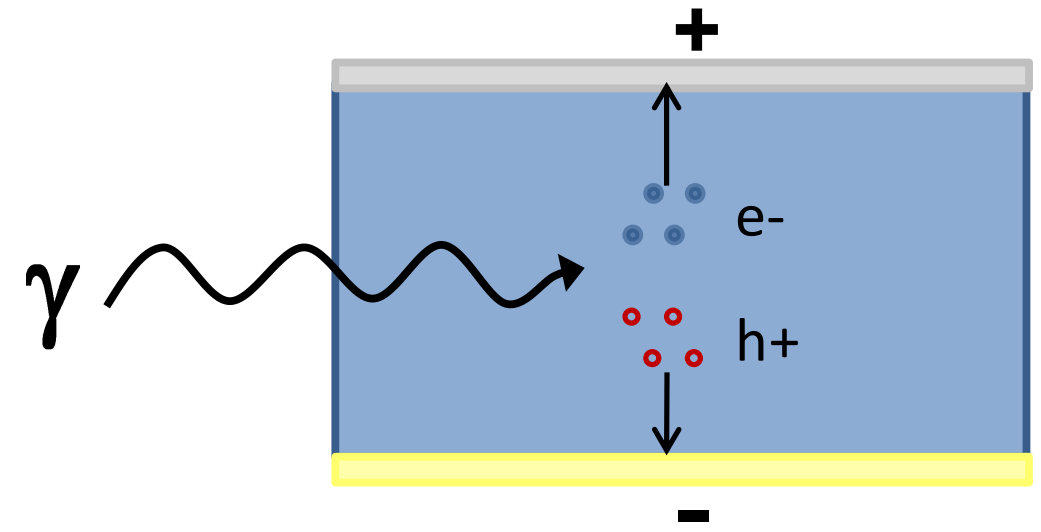

Figure 2.3 Typical Gamma Ray Structure Indicating Gamma-Generated Carriers 
When the detector material absorbs a gamma ray, it creates a cloud of electron-hole pairs; these carriers induce mirror charges at the electrodes. As the electrons and holes are swept in opposite directions by the high electric field, they couple more strongly to the closer electrode (electrons to the positive electrode and holes to the negative electrode). The induced current flows into the positive electrode as soon as the generated charge begins to move, creating a signal immediately rather than when generated carriers reach the electrodes [7]. The simplified Hecht relation in equation 2.3 describes charge collection efficiency for carriers generated at the center of a single carrier system [8].

$$
\eta=\frac{\mu \tau V}{L^{2}}\left[1-\exp \left(-\frac{L^{2}}{\mu \tau V}\right)\right]
$$

Increasing the mobility-lifetime product, $\mu \tau$, of the detector material or increasing voltage, $\mathrm{V}$, will increase charge collection, since generated charge will travel farther before it recombines, inducing more signal current. Increasing the device thickness, L, decreases charge collection efficiency, since induced charge is inversely proportional to the square of distance. Figure 2.4 shows the charge collection efficiency versus bias voltage for the $1 \mathrm{~cm}$ thick device mentioned above; this figure uses a two-carrier model to illustrate the difference between electron (e-) and hole $(\mathrm{h}+)$ collection 


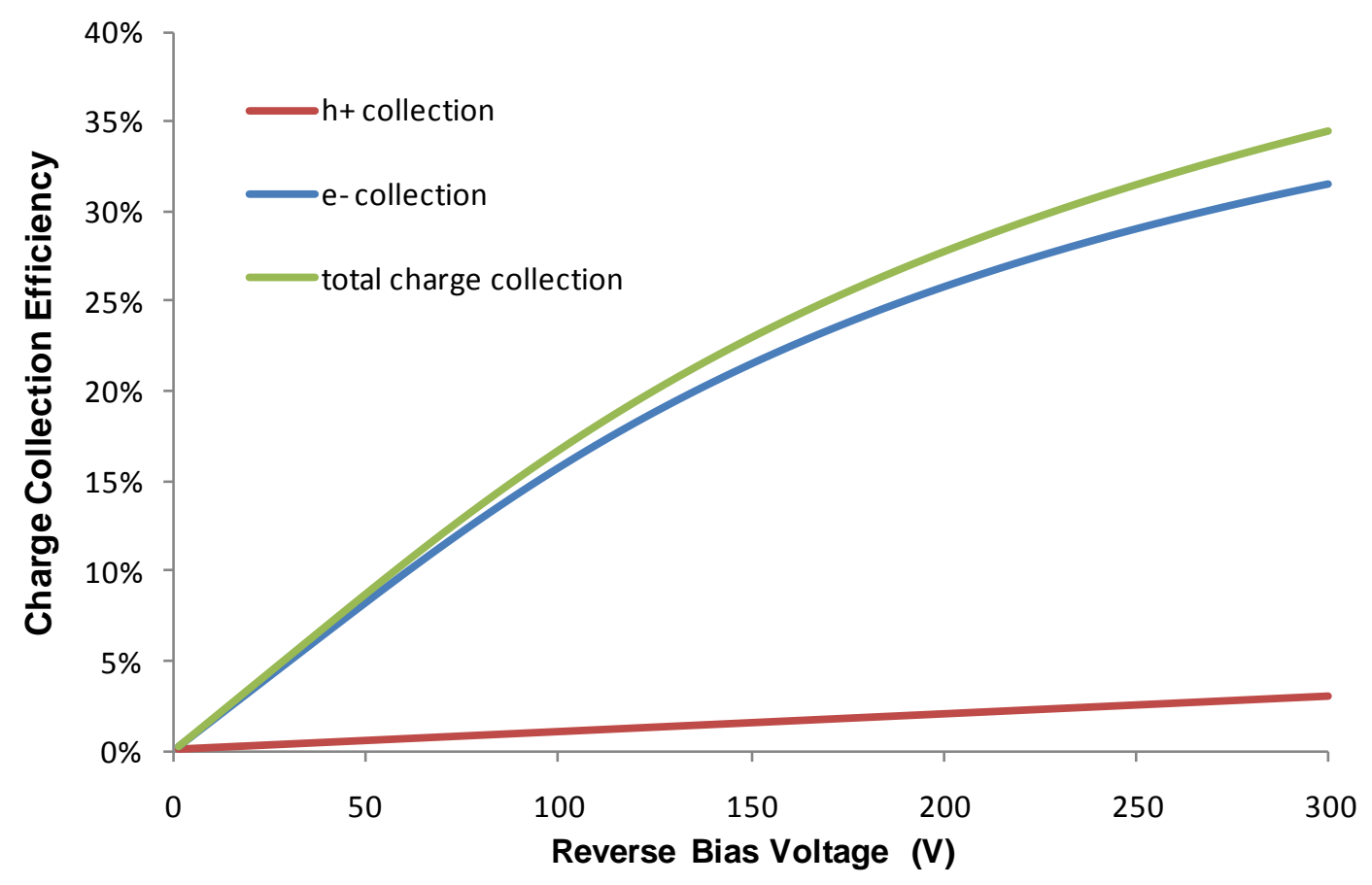

Figure 2.4 Charge Collection in $1 \mathrm{~cm}$ Thick CdTe Detector; $\mu_{\mathrm{e}} \tau_{\mathrm{e}} \approx 1 \mathrm{~ms}, \mu_{\mathrm{h}} \tau_{\mathrm{h}} \approx 0.1 \mathrm{~ms}$

Notice that electrons dominate total charge collection, since their mobility-lifetime product, $\mu_{\mathrm{e}} \tau_{\mathrm{e}}$, is more than an order of magnitude greater than the hole mobility-lifetime product in CdTe [9]. Typically the device would be biased in excess of $500 \mathrm{~V}$, to increase charge collection [4].

Although high voltage improves charge collection, it also increases leakage current; shot noise from discrete carriers and 1/f noise typically from trapping, both increase with leakage current:

$$
\begin{gathered}
I_{\text {shot }}{ }^{2} \propto I_{\text {leak }} \\
I_{1 / f}{ }^{2} \cong K_{f}\left(A J_{\text {leak }}\right)^{2}
\end{gathered}
$$

where $\mathrm{I}_{\text {leak }}$ is the leakage current, $\mathrm{I}_{\text {shot }}$ relates to shot noise, and $\mathrm{I}_{1 / \mathrm{f}}$ relates to $1 / \mathrm{f}$ noise. This noise adds in quadrature with many other independent noise sources: thermal noise from contact resistance, noise from shaping time and capacitance in detector electronics, 
and noise from material non-uniformity [4]. However, shot and 1/f noise dominate at lower gamma ray energies. Figure 2.5 shows energy resolution as a result of leakage current for a $200 \mathrm{keV}$ gamma ray.

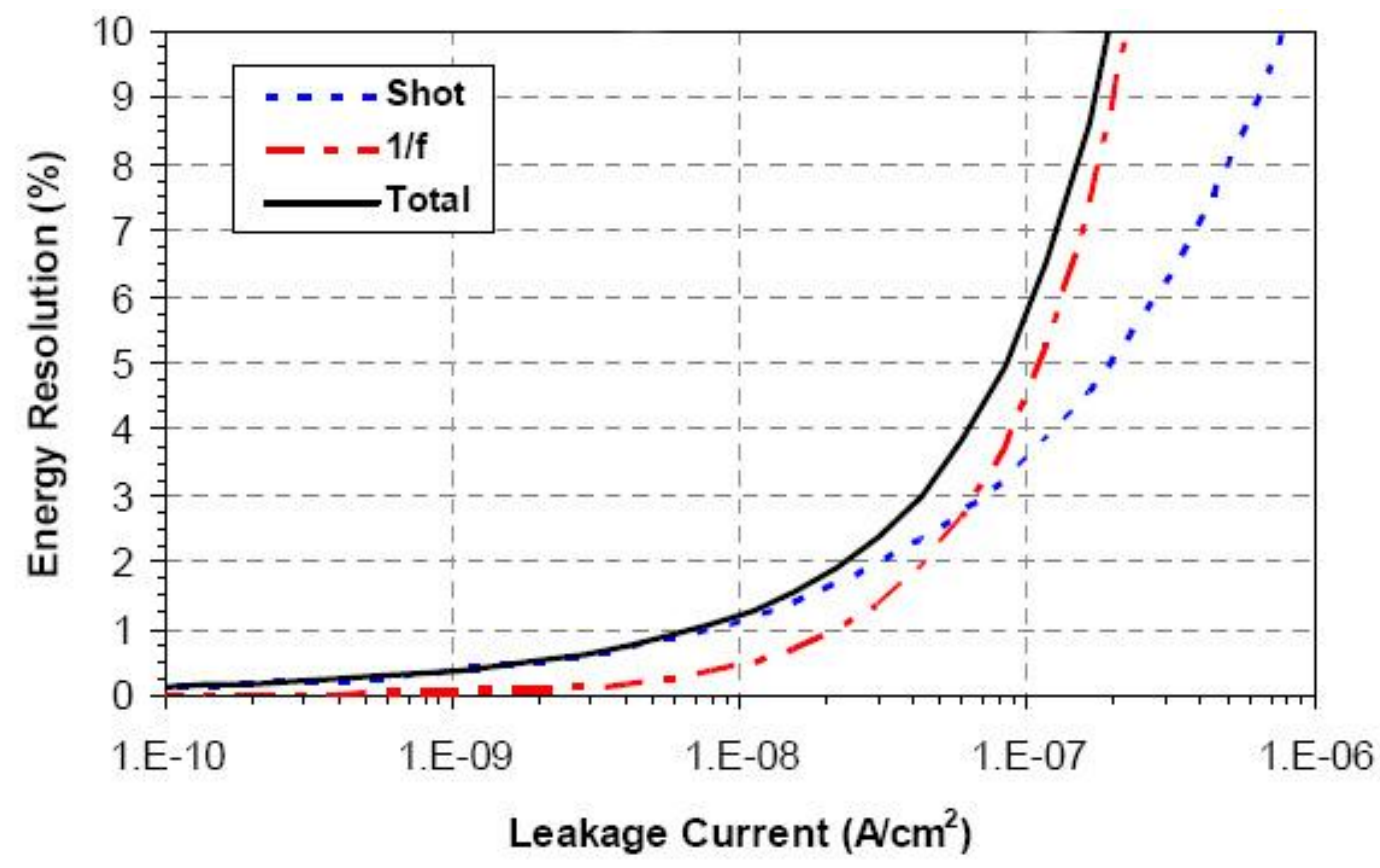

Figure 2.5 Energy Resolution Dependence on Leakage Current for a $200 \mathrm{keV}$ Gamma Ray [4]

Figure 2.4 indicates that leakage current less than $10 \mathrm{nA} / \mathrm{cm}^{2}$ should provide the desired $1 \%$ energy resolution, but the other noise sources will degrade this resolution, especially at higher gamma ray energies [4].

Gamma ray absorption, charge collection, and detector noise all receive conflicting performance benefits from detector characteristics. High bias increases charge collection efficiency but also increases leakage current, thus increasing noise. Higher resistivity decreases leakage current in an ohmic device:

$$
J_{\text {leak }}=\frac{V}{\rho L}
$$


where $\mathrm{V}$ is the reverse bias, $\rho$ is resistivity, and $\mathrm{L}$ is device thickness. However, increasing material resistivity typically decreases carrier lifetime and therefore the $\mu \tau$ product, thus reducing charge collection efficiency again. Thicker detectors absorb more incident gamma rays and have lower leakage, but also have lower charge collection efficiency. Determining the optimal combination between these factors is important in a final detector design.

\subsection{High Resistivity Cadmium Telluride}

Cadmium Telluride (CdTe) forms a Zincblende lattice with $2.94 \times 10^{22}$ atoms $/ \mathrm{cm}^{3}$ [10]. The Zincblende structure is two interpenetrating face-centered cubic (FCC) structures with $\mathrm{Cd}$ at $(0,0,0)$ and Te at $(1 / 4,1 / 4,1 / 4)$, as shown in figure 2.6 [11].

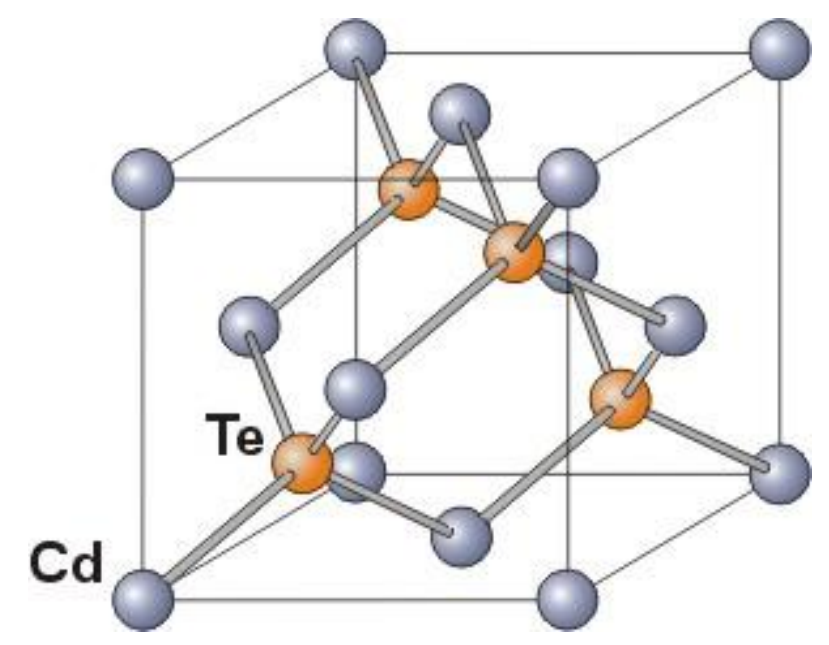

Figure 2.6 CdTe Zincblende Structure [12]

Wafers cut along the (111) plane are used for gamma detectors, probably due to better crystal growth in this orientation. Since the (111) surface is terminated with one type of atom, there is an A-face $(\mathrm{Cd})$ and a B-face $(\mathrm{Te})$ on the wafers. Figure 2.7 shows the orientation of atoms on the (111) surface; the size difference between $\mathrm{Cd}$ and $\mathrm{Te}$ is 
exaggerated. The different stoichiometry of the two faces leads to differences in native oxide growth, surface defect density, and contact formation [13].

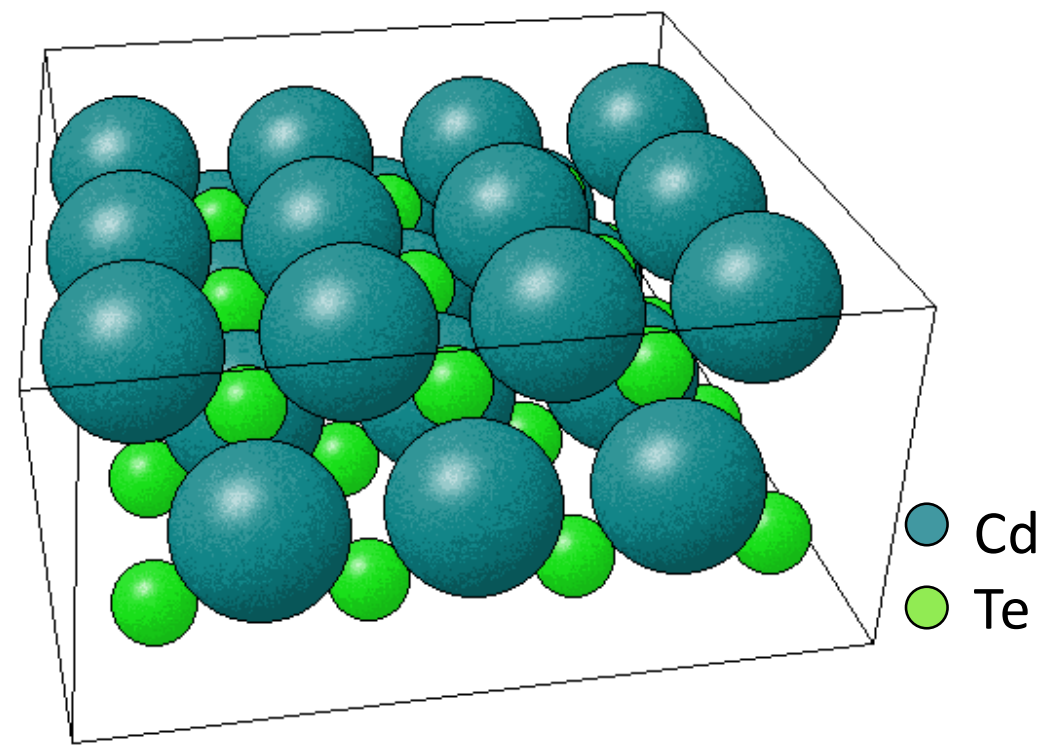

Figure 2.7 Wafer Surface Along (111) Plane [14]

The gamma detectors studied in this thesis are built from $1 \mathrm{~mm}$ thick (111) p-CdTe wafers from Acrorad. The boules are grown by Travelling Heater Method (THM) and chlorine $(\mathrm{Cl})$ doped to compensate defects. These defects are typically cadmium vacancies, a missing $\mathrm{Cd}$ atom, and tellurium antisite defects, a $\mathrm{Cd}$ atom occupying a Te site, which produce deep trap levels that control material resistivity [15]. Without compensation these traps would produce too many carriers to maintain the resistivity, $\rho>10^{9} \Omega \cdot \mathrm{cm}$; this is the only material parameter specified by Acrorad. 


\section{INITIAL DESIGN}

The previous chapter discussed the importance of high detector bias for charge collection and the problems caused by the corresponding leakage current. Increasing material resistivity is one path to decreasing leakage current. However, this increased resistivity often is accompanied by a decreased carrier mobility-lifetime product, $\mu \tau$, which reduces charge collection efficiency; since higher resistivity is achieved through more trap compensation, and more impurities yields more scattering and lower mobility.

Replacing one ohmic contact with a Schottky contact will reduce the reverse current without requiring increased material resistivity and decreased $\mu \tau$; the Schottky diode characteristic in figure 3.1 shows an example of reduced leakage current due to rectification. 


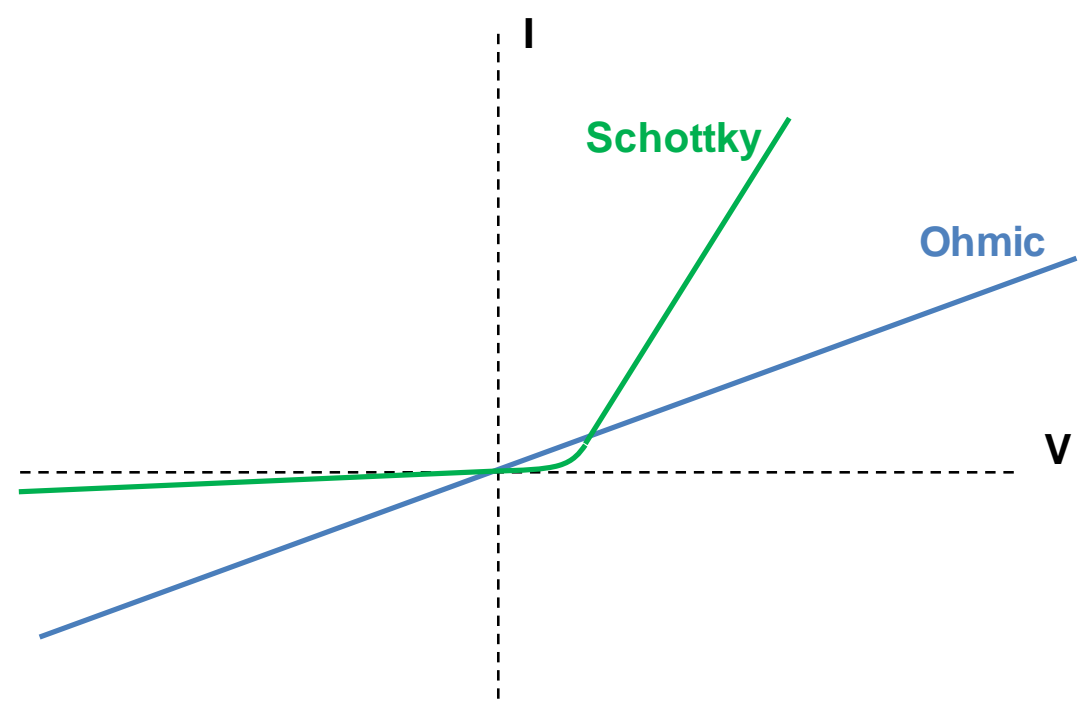

Figure 3.1 Ohmic and Schottky Detector I-V Characteristics

Figure 3.2 indicates how the Schottky contact blocks reverse leakage current, without blocking gamma-generated carriers.

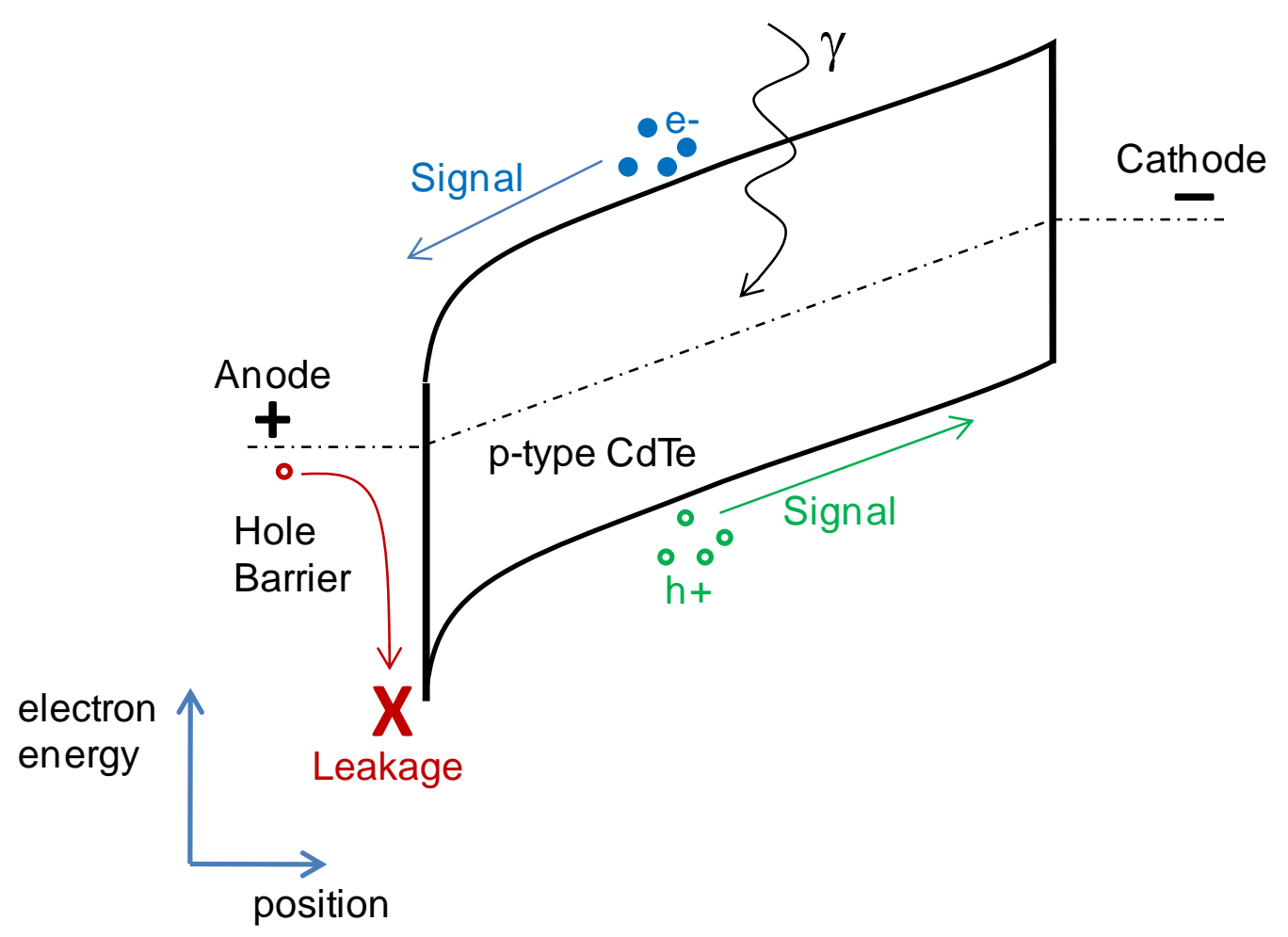

Figure 3.2 Schottky Detector Concept 
The applied bias in figure 3.2 sweeps generated signal carriers towards low semiconductor to metal barriers, but the high potential barrier for holes blocks the metalsemiconductor leakage current at the anode [4]. The following sections develop initial models for Schottky and ohmic detectors, evaluate their performance, and propose a physical device design.

\subsection{Silvaco Device Simulator}

The gamma ray detectors were numerically modeled using the Silvaco Atlas physics simulator. The basic device model utilizes fundamental semiconductor relations between electrostatic potential and carrier densities. Poisson's equation relates the fixed charge, electric field, and potential:

$$
\nabla^{2} \varphi=\nabla \cdot \boldsymbol{E}=\frac{\rho}{\varepsilon}
$$

where $\varphi$ is electrostatic potential, $E$ is electric field vector, $\rho$ is fixed charge density, and $\varepsilon$ is the semiconductor permittivity. Carrier continuity equations describe carrier densities in terms of transport, generation, and recombination:

$$
\begin{gathered}
\frac{\partial n}{\partial t}=\frac{1}{q} \nabla \cdot \boldsymbol{J}_{\boldsymbol{n}}+G_{n}-R_{n} \\
\frac{\partial p}{\partial t}=-\frac{1}{q} \nabla \cdot \boldsymbol{J}_{\boldsymbol{p}}+G_{p}-R_{p}
\end{gathered}
$$

where $\mathrm{G}$ and $\mathrm{R}$ are the generation and recombination rates, specified separately for holes and electrons. The basic transport model is the drift-diffusion theory:

$$
\begin{aligned}
& \boldsymbol{J}_{\boldsymbol{n}}=-q \mu_{n} n \boldsymbol{\nabla} \phi_{n}=q \mu_{n} n \boldsymbol{E}+q D_{n} \boldsymbol{\nabla n} \\
& \boldsymbol{J}_{\boldsymbol{p}}=-q \mu_{p} \mathrm{p} \boldsymbol{\nabla} \phi_{p}=q \mu_{p} p \boldsymbol{E}+q D_{p} \boldsymbol{\nabla} \mathrm{p}
\end{aligned}
$$


where $\mu$ is mobility, $\phi$ is the quasi-Fermi level, $\mathrm{n}$ and $\mathrm{p}$ are carrier concentrations, and the diffusion coefficient is $D=\mu_{n} k T / q$. These coupled differential equations are discretized and solved self consistently on a finite element grid. Atlas also includes more complex models which will be utilized in later modeling [16], [17].

\subsection{Detector Design}

Atlas has an existing CdTe model which serves as a basis for the bulk material in the simulation, but most material parameters are modified based on the literature; table 3.1 shows the material parameter values.

Table 3.1 User-Defined Material Parameters for CdTe at 300K

\begin{tabular}{|l|ll|}
\hline Bandgap & $1.50 \mathrm{eV}$ & {$[16],[18]$} \\
\hline Electron Effective Mass, $m_{n}^{*} / m_{0}$ & 0.1 & {$[19],[20]$} \\
\hline Hole Effective Mass, $m_{p}^{*} / m_{0}$ & 0.4 & {$[20]$} \\
\hline Electron Affinity, $\chi$ & $4.28 \mathrm{eV}$ & {$[16]$} \\
\hline Electron Mobility, $\mu_{\mathrm{n}}$ & $1077 \mathrm{~cm}^{2} / \mathrm{V} \cdot \mathrm{s}$ & {$[21]$} \\
\hline Hole Mobility, $\mu_{\mathrm{p}}$ & $80 \mathrm{~cm}^{2} / \mathrm{V} \cdot \mathrm{s}$ & {$[21]$} \\
\hline Electron Lifetime, $\tau_{\mathrm{n}}$ & $3 \mu \mathrm{s}$ & {$[9],[18]$} \\
\hline Hole Lifetime, $\tau_{\mathrm{p}}$ & $2 \mu \mathrm{s}$ & {$[9],[18]$} \\
\hline Relative Permittivity, $\varepsilon_{\mathrm{r}}$ & 10 & {$[16],[19],[22]$} \\
\hline Acceptor Dopant Concentration, $\mathrm{N}_{\mathrm{A}}$ & $7 \times 10^{7} \mathrm{~cm}^{-3}$ & \\
\hline
\end{tabular}

The acceptor doping concentration, $\mathrm{N}_{\mathrm{A}}$, is calculated from the known resistivity, $\rho>10^{9} \Omega \cdot \mathrm{cm}$. Since the material is p-type, the hole concentration is equal to the acceptor doping concentration.

$$
\rho=\left[q\left(\mu_{n} n+\mu_{p} p\right)\right]^{-1} \Rightarrow p=N_{A}=\frac{1}{q \mu_{p} \rho}
$$


Figure 3.3 shows electron and hole mobility for Acrorad Cl-compensated CdTe; at low temperature the mobility is trap controlled, but at higher temperatures ionized impurity and polar optical scattering determine the mobility [21].

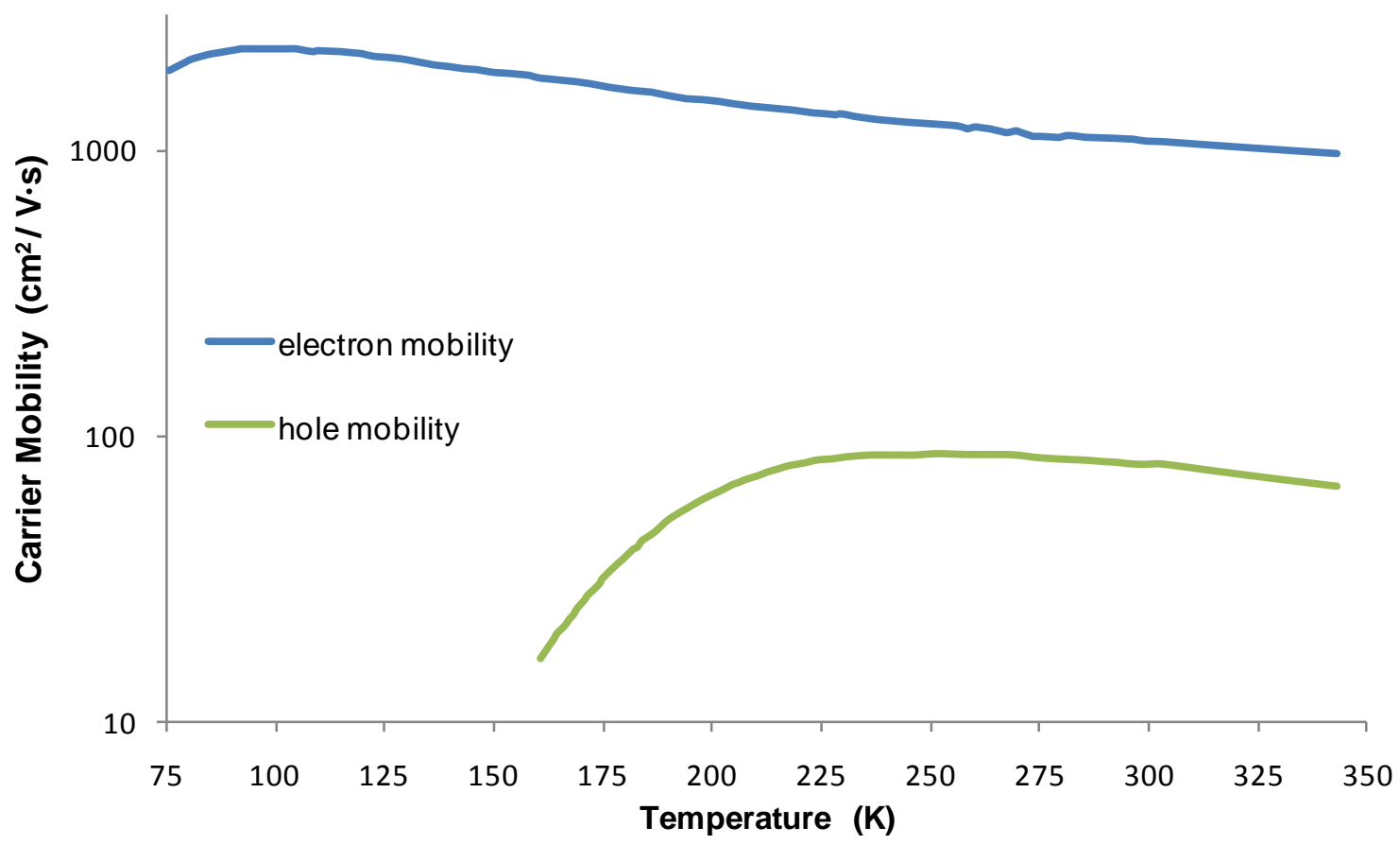

Figure 3.3 Electron and Hole Mobility Versus Temperature [21]

Equation 3.7 models the mobility over our measured range $(294 \mathrm{~K}-345 \mathrm{~K})$, and the mobility model parameters extracted from figure 3.3 are given in table 3.2.

$$
\mu=\mu_{300}\left(\frac{T}{300}\right)^{-\tau_{\mu}}
$$

Table 3.2 Mobility Temperature Dependence Model

\begin{tabular}{|c|c|c|c|}
\hline$\mu_{\mathrm{n} 300}$ & $\tau_{\mu \mathrm{n}}$ & $\mu_{\mathrm{p} 300}$ & $\tau_{\mu \mathrm{p}}$ \\
\hline $1077 \mathrm{~cm}^{2} / \mathrm{V} \cdot \mathrm{s}$ & 0.78 & $80 \mathrm{~cm}^{2} / \mathrm{V} \cdot \mathrm{s}$ & 1.44 \\
\hline
\end{tabular}

In an ohmic detector, the contacts are identical and should minimally affect conduction. Figure 3.4 shows the simulated detector structure, and figure 3.5 shows the 
ohmic detector band diagram in a two-dimensional cut; in the ohmic device, the surface Fermi level equals the bulk Fermi level. Figure 3.6 shows the corresponding current versus voltage (I-V) characteristic.

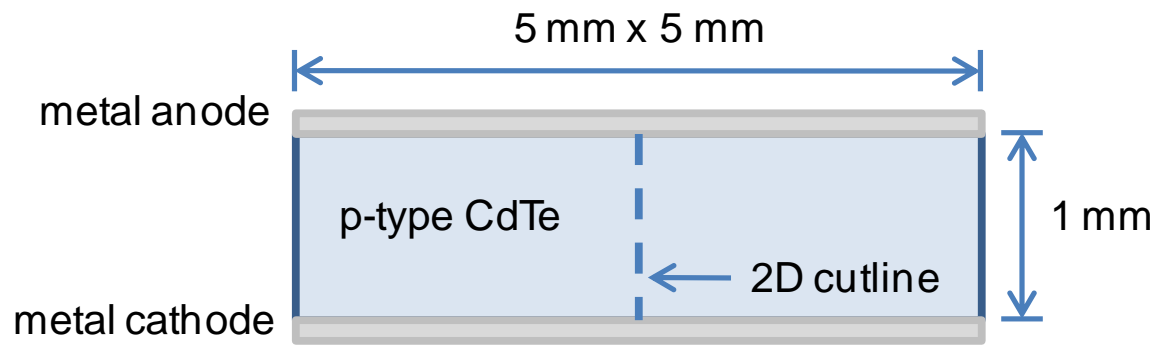

Figure 3.4 Simulated Detector Structure

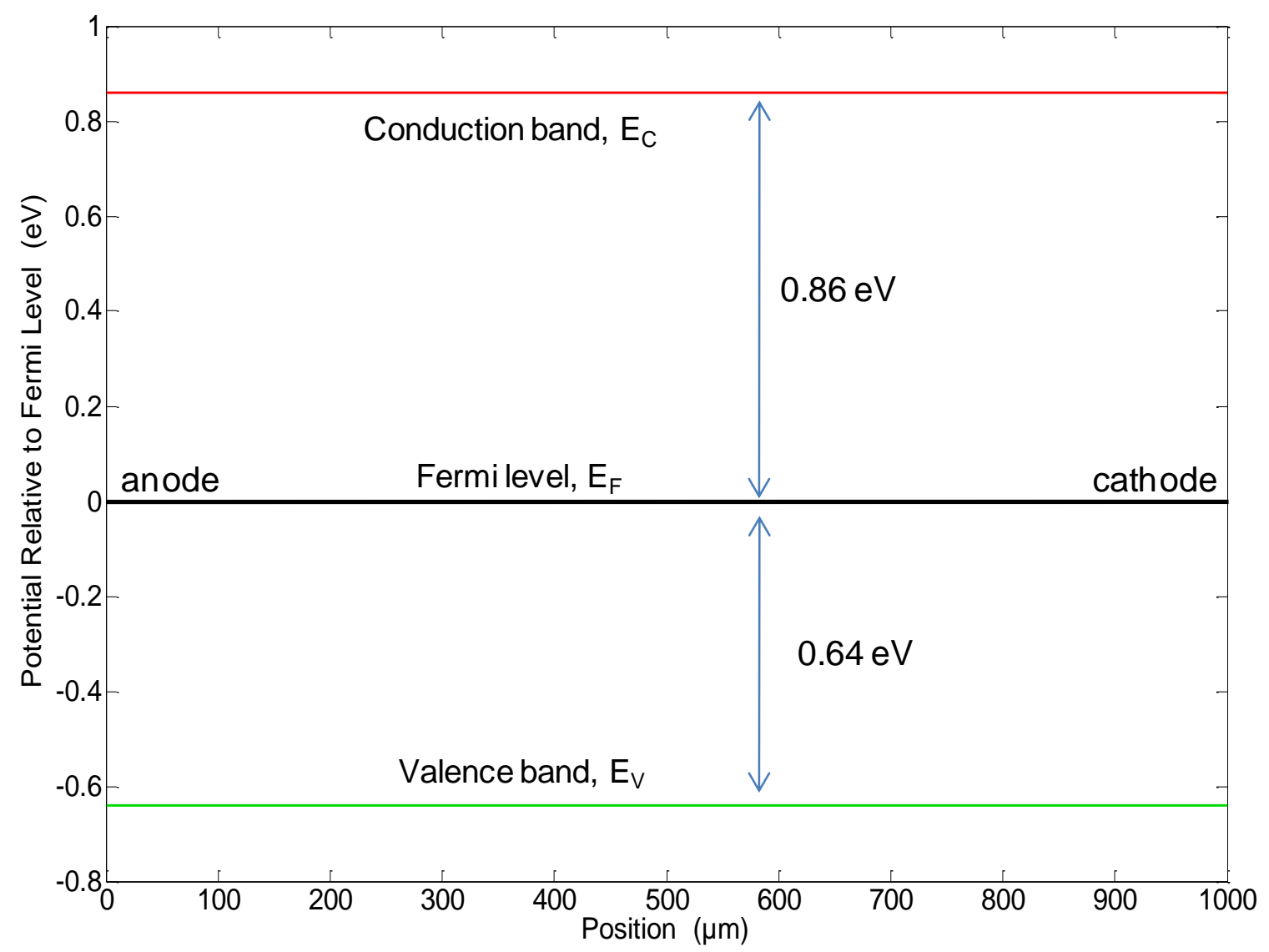

Figure 3.5 Simulated Ohmic Detector Energy Band Diagram 


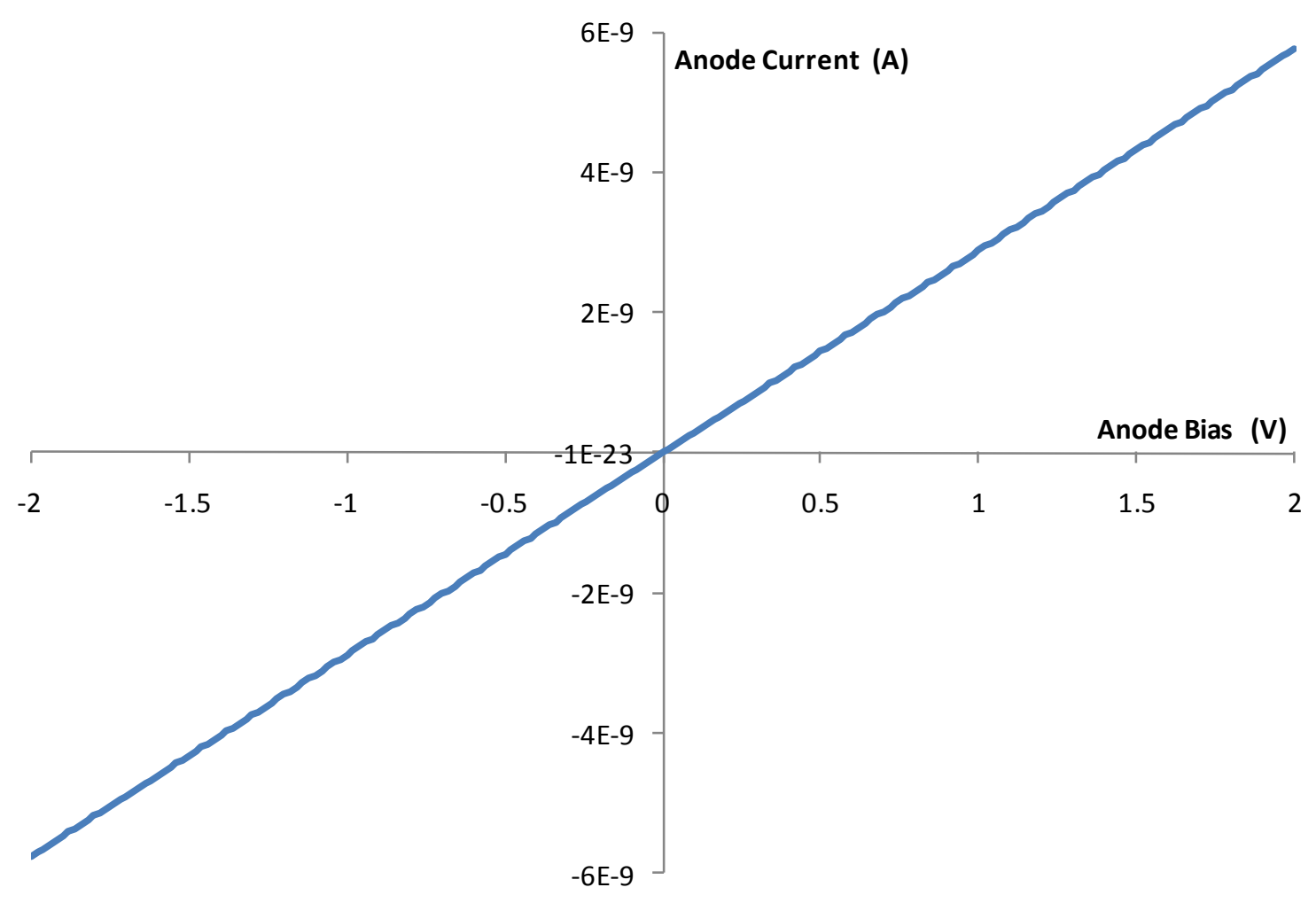

Figure 3.6 Simulated Ohmic Detector I-V Characteristic

Figure 3.7 defines energy levels, barrier heights, and accumulation regions. A hole barrier, $\phi_{\mathrm{Bp}}$, at the anode should block hole current in reverse bias, as shown previously in figure 3.2.

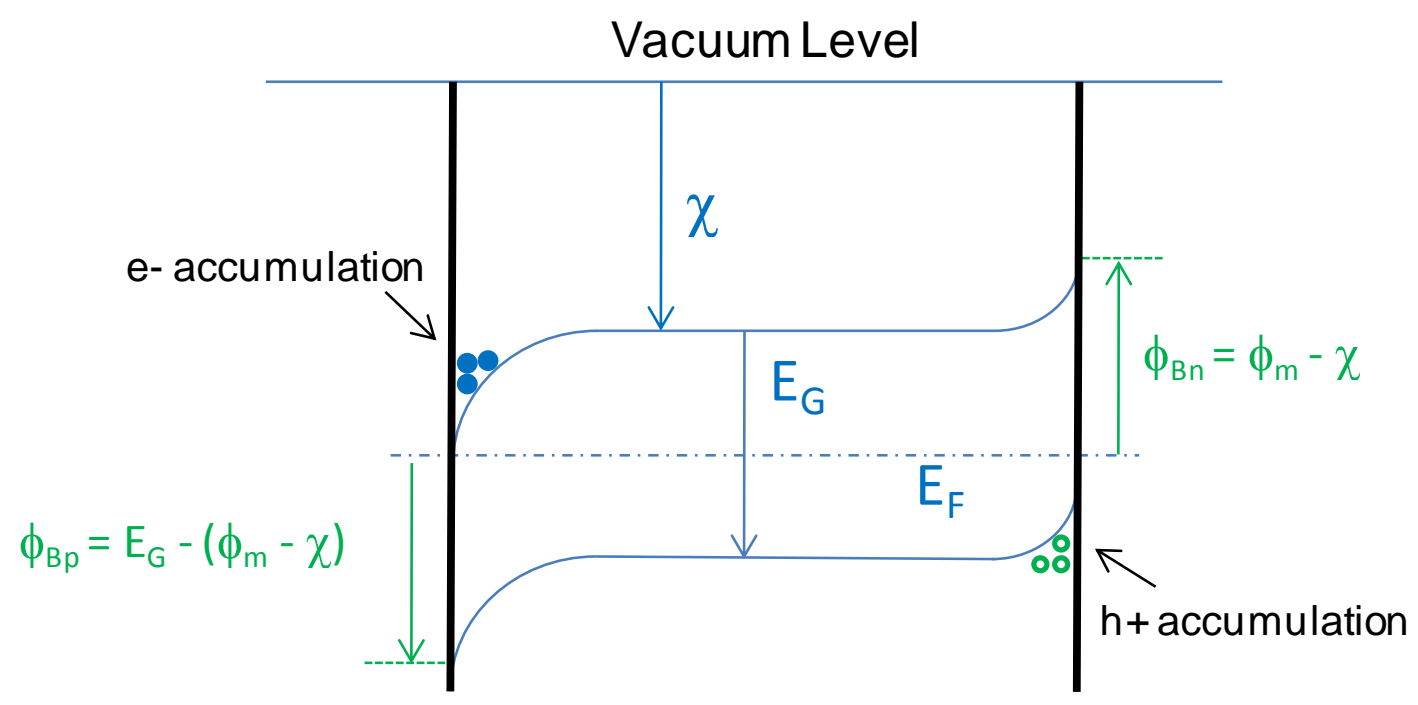

Figure 3.7 Schottky Barrier Height Definition 
A low work function contact creates a large hole barrier, so the anode metal work function, $\phi_{\mathrm{m}}$, was varied from $5.00 \mathrm{eV}>\phi_{\mathrm{m}}>4.8 \mathrm{eV}$ in the simulation; this yields a hole barrier between $0.78 \mathrm{eV}>\phi_{\mathrm{m}}>0.98 \mathrm{eV}$. The cathode contact presents a few choices: it could be another hole barrier, an electron barrier, or set to the CdTe bulk Fermi level. A large hole barrier decreases hole current but increases electron current; with small electron barriers and large hole barriers at each contact, electron injection could dominate, and the hole barriers would not control the reverse current. This back-to-back Schottky diode configuration is also difficult to characterize electrically. Setting the cathode work function equal to the bulk Fermi level also presents the problem of dominant electron current in reverse bias due to minority carrier injection. A large electron barrier at the cathode will minimize current due to electron injection, as shown in figure 3.8; this should allow the anode hole barrier to control the reverse bias leakage current.
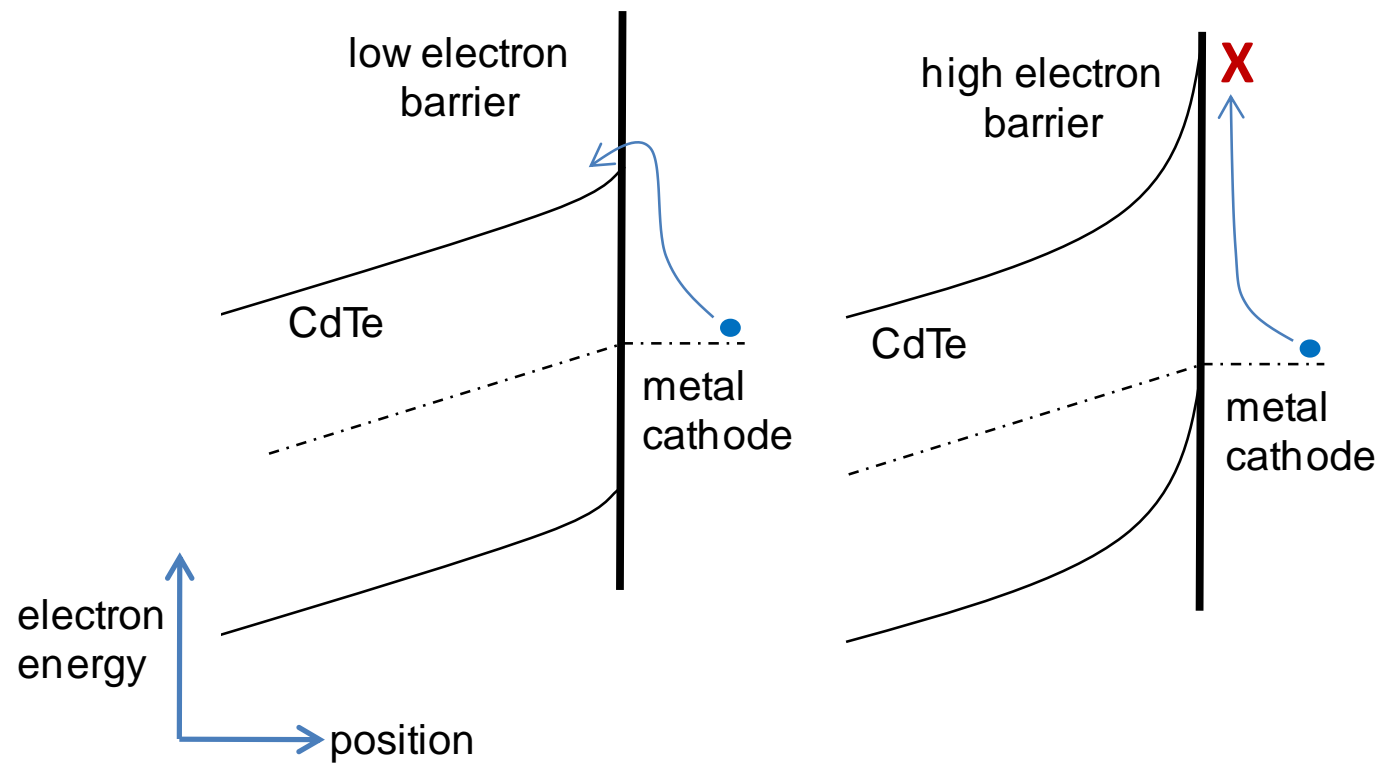

Figure 3.8 Schottky Detector Reverse Bias Electron Injection 
A high work function cathode creates an low hole barrier and a high electron barrier, so the cathode work function was set to $\phi_{\mathrm{m}}=5.35 \mathrm{eV}$, yielding an electron barrier $\phi_{\mathrm{Bn}}=1.18 \mathrm{eV}$. Figure 3.9 shows the energy band diagrams for the simulated Schottky detectors.

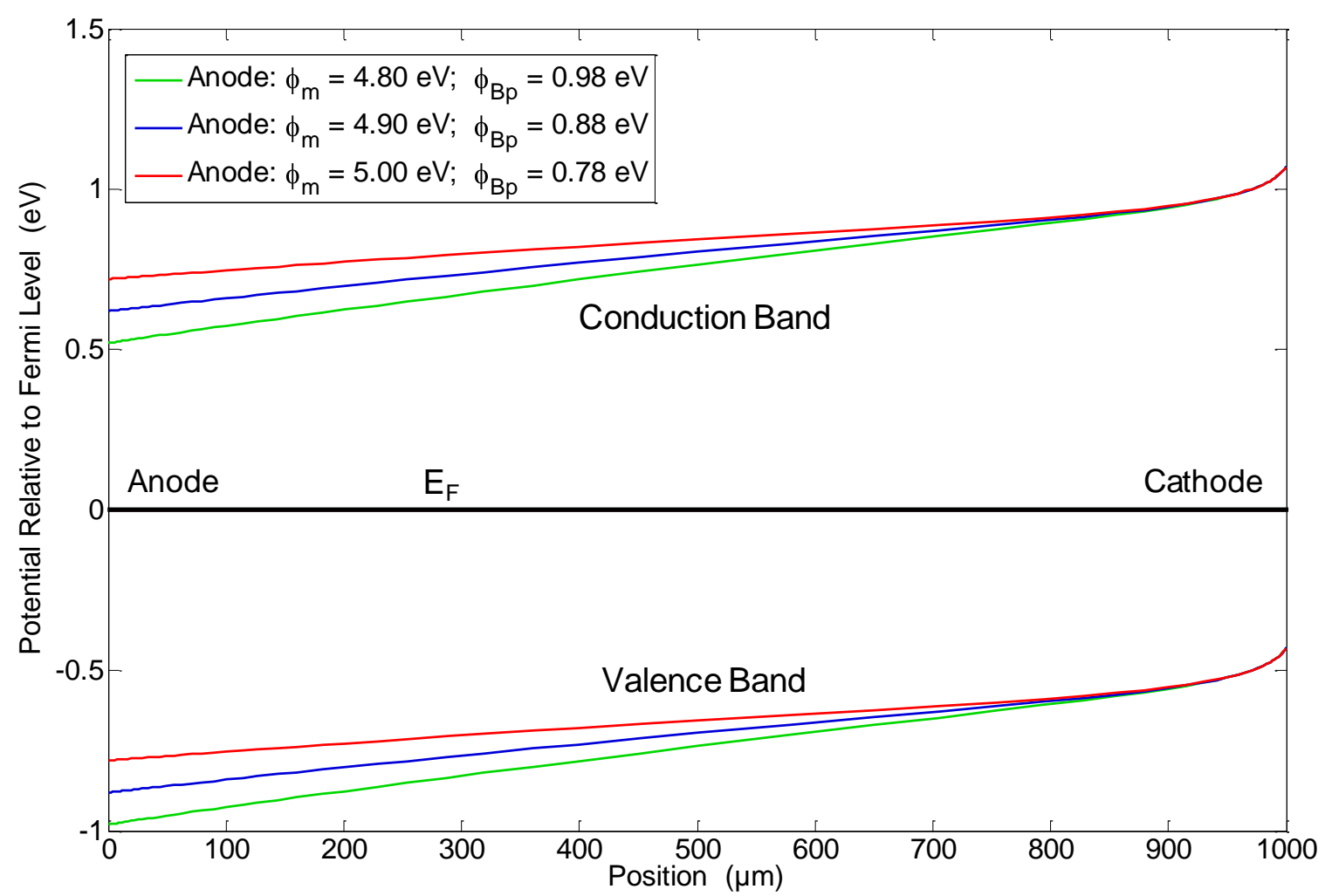

Figure 3.9 Simulated Schottky Detector Energy Band Diagrams

The constant slope in figure 3.9 indicates that the device is fully depleted, due to the large contact potentials and the low doping concentration, $\mathrm{N}_{\mathrm{A}}=7 \times 10^{7} \mathrm{~cm}^{-3}$.

In diodes there is always a potential drop from the low work function contact to the high work function contact; in this case, the potential drop is from anode to cathode. Applying a negative bias to the anode overcomes the built-in potential, pushing the device into forward bias. Placing the lower work function at the cathode would reverse 
the device polarity, yielding the conventional forward bias direction. The detector diode is designed to have a Schottky hole barrier at the anode (low work function), so negative anode bias forward biases the diode, and positive anode bias reverse biases the diode; this bias polarity does not change for the remainder of this thesis. Figure 3.10 shows the Schottky current-voltage characteristics and the current-voltage characteristic for the ohmic detector. The increased forward current at anode work function $\phi_{\mathrm{m}}=4.80 \mathrm{eV}$ is due to electron injection at the anode.

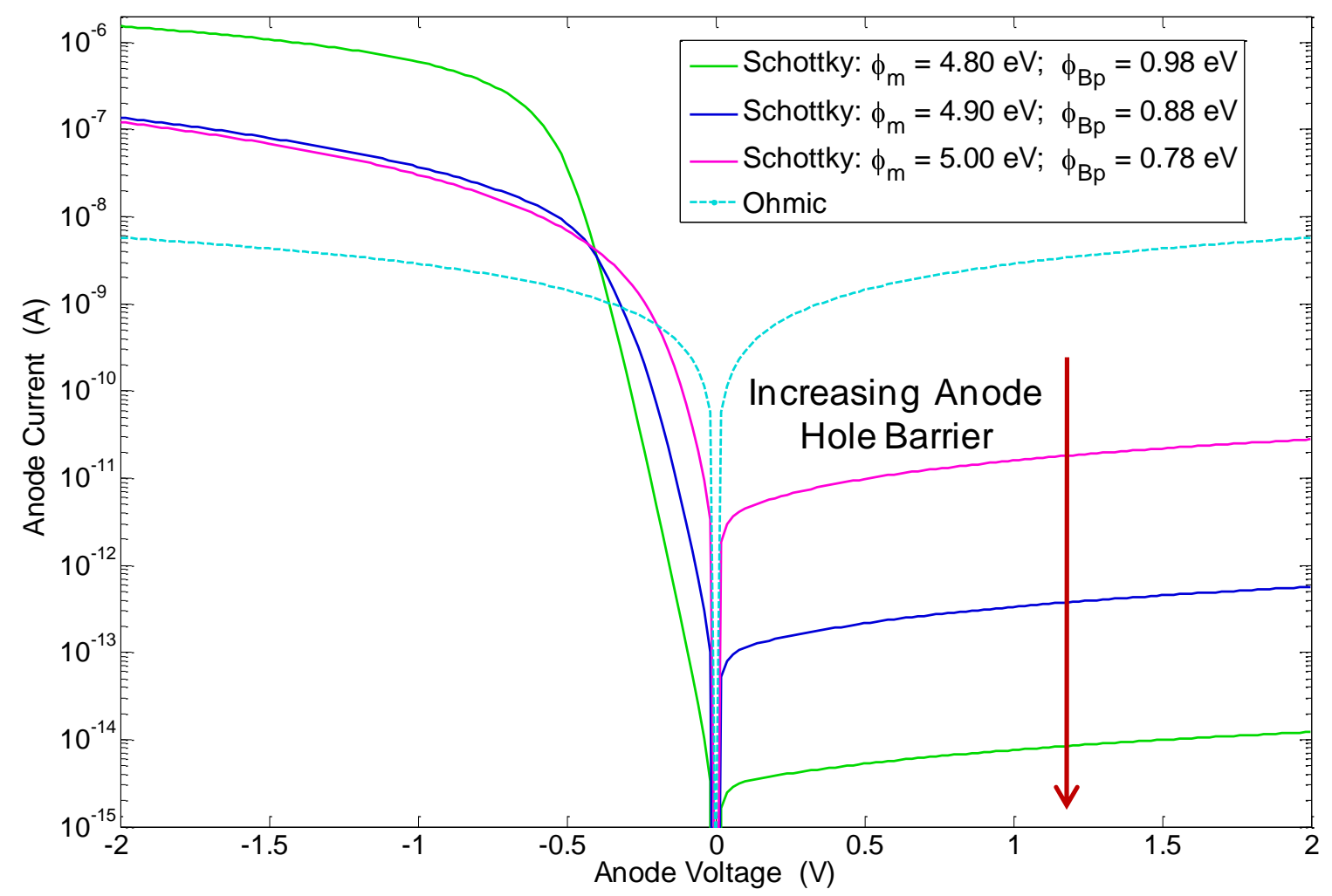

Figure 3.10 Schottky and Ohmic Detector Current-Voltage Characteristics

The simulations confirm that a high anode hole barrier and a high cathode electron barrier yield a detector with the lowest reverse leakage; the reverse leakage is also tunable by increasing the anode hole barrier, as shown in figures 3.10 and 3.11. 


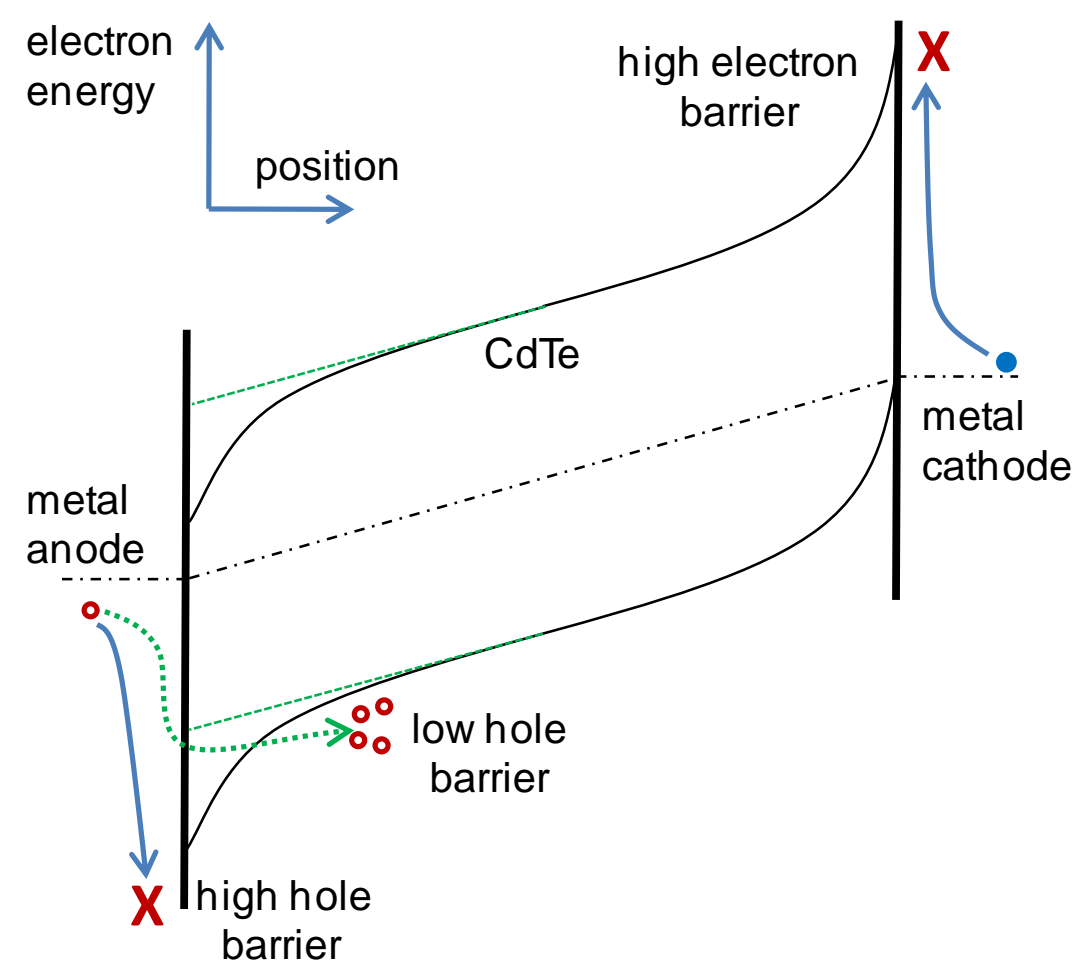

Figure 3.11 Schottky Detector Reverse Bias Conduction

\subsection{Physical Device Design}

Previously fabricated ohmic detectors include p-CdTe with two platinum contacts (Pt-CdTe-Pt) and p-CdTe with two gold contacts (Au-CdTe-Au). Average work functions for Pt and Au metal are $5.65 \mathrm{eV}$ and $5.35 \mathrm{eV}$, respectively [23]. Simulations in the previous section show that these metals should form ohmic contacts to p-CdTe, and this agrees with results in the literature [24], [25]. Gold was chosen for the ohmic cathode contact in this device set, due to reliability in past fabrication and measurement. For the Schottky anode, aluminum was chosen to create a large hole barrier; an average work function for $\mathrm{Al}$ metal is $4.15 \mathrm{eV}$ [23]. The ohmic contact can be ignored in initial modeling, allowing simpler analysis of the single Schottky contact. 


\section{DEVICE FABRICATION AND MEASUREMENT}

Two unique $5 \mathrm{~mm} \times 5 \mathrm{~mm}$ samples were fabricated: one received an argon ion sputter etch under the Schottky contact and the other did not; figure 4.1 shows this process. Sputtering CdTe with energetic argon ions is known to remove the native oxide, decrease surface defect density, and modify surface stoichiometry [26]

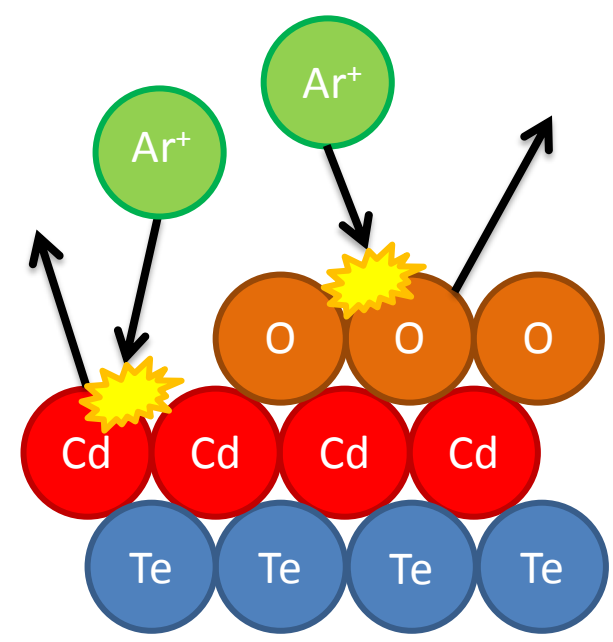

Figure 4.1 Argon Ion Sputter Etch Process; Courtesy: L. F. Voss

Lower surface defect density should improve Schottky barrier formation, and yield lower leakage current. In attempt to isolate the Schottky contact as the only new variable, these devices are fabricated with the same methods as prior ohmic devices. The devices are 
characterized with current-voltage measurements over a low and high voltage range, so this chapter analyzes the measurement system to evaluate measurement accuracy.

\subsection{Fabrication}

Before depositing each contact, the CdTe wafer was exposed to ozone for 3 minutes and etched for 10 seconds with buffered oxide etch (BOE), a combination of ammonium fluoride $\left(\mathrm{NH}_{4} \mathrm{~F}\right)$ and hydrofluoric acid (HF) [27]. The B-face of the wafer received an argon ion $(\mathrm{Ar}+)$ sputter etch at $750 \mathrm{~W}$ for 1 minute. Approximately $2500 \AA$ of gold $(\mathrm{Au})$ was sputter deposited to form an un-patterned (blanket) cathode contact on the B-face. The A-face of one sample was sputter etched with Ar+ at $750 \mathrm{~W}$ for 1 minute, and the other sample did not receive a sputter etch on the A-face. The A-face of each device was patterned with photoresist, sputtered with $2500 \AA$ of aluminum (Al), and the excess Al was removed by lift-off. For the remainder of this paper, the devices are differentiated by whether or not the A-face received a sputter etch before Al anode deposition: one device will be referred to as the "no sputter etch device" and the other as the "sputter etch device". Table 4.1 lists the fabricated device sizes, and figure 4.2 shows the patterned anode contact geometry.

Table 4.1 Device Geometry

\begin{tabular}{|c|c|c|}
\hline Anode Diameter & Gap Width & Guard Ring Width \\
\hline $500 \mu \mathrm{m}$ & $25 \mu \mathrm{m}$ & $50 \mu \mathrm{m}$ \\
\hline $250 \mu \mathrm{m}$ & $25 \mu \mathrm{m}$ & $50 \mu \mathrm{m}$ \\
\hline
\end{tabular}




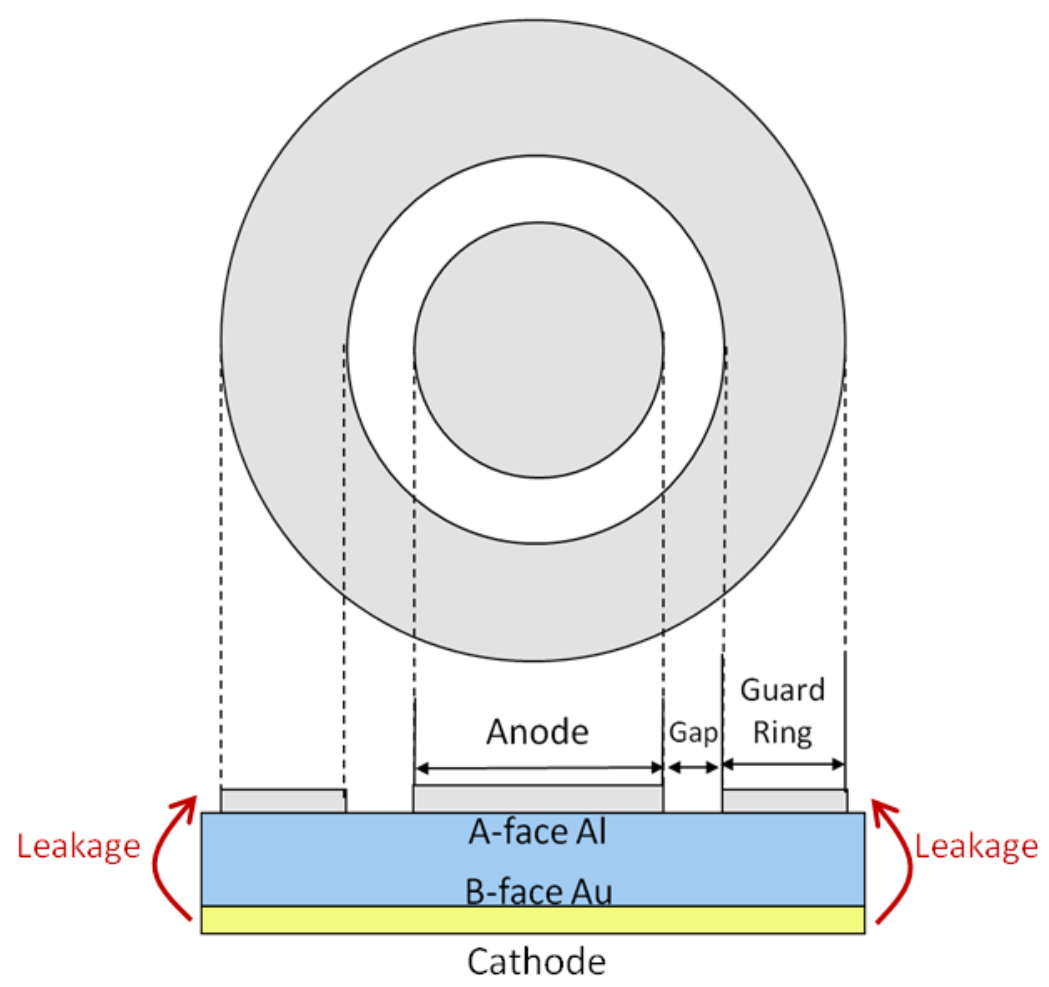

Figure 4.2 Anode Contact Geometry (Cathode Not to Scale)

During measurements, the guard ring is placed at the same potential as the anode, so no current travels between the anode and guard ring. The guard ring collects current that leaks along the device surface from the cathode, as shown in figure 4.2. This is a very significant effect and severely degrades detector performance, since this path is much less resistive than the bulk material in reverse bias. Figure 4.3 shows the collected anode and guard ring currents for a typical device at high voltages. Notice as the anode bias approaches $200 \mathrm{~V}$ the guard ring shows breakdown in the surface current, but the center electrode detects only the current through the device, shielded from surface currents by the guard ring. 


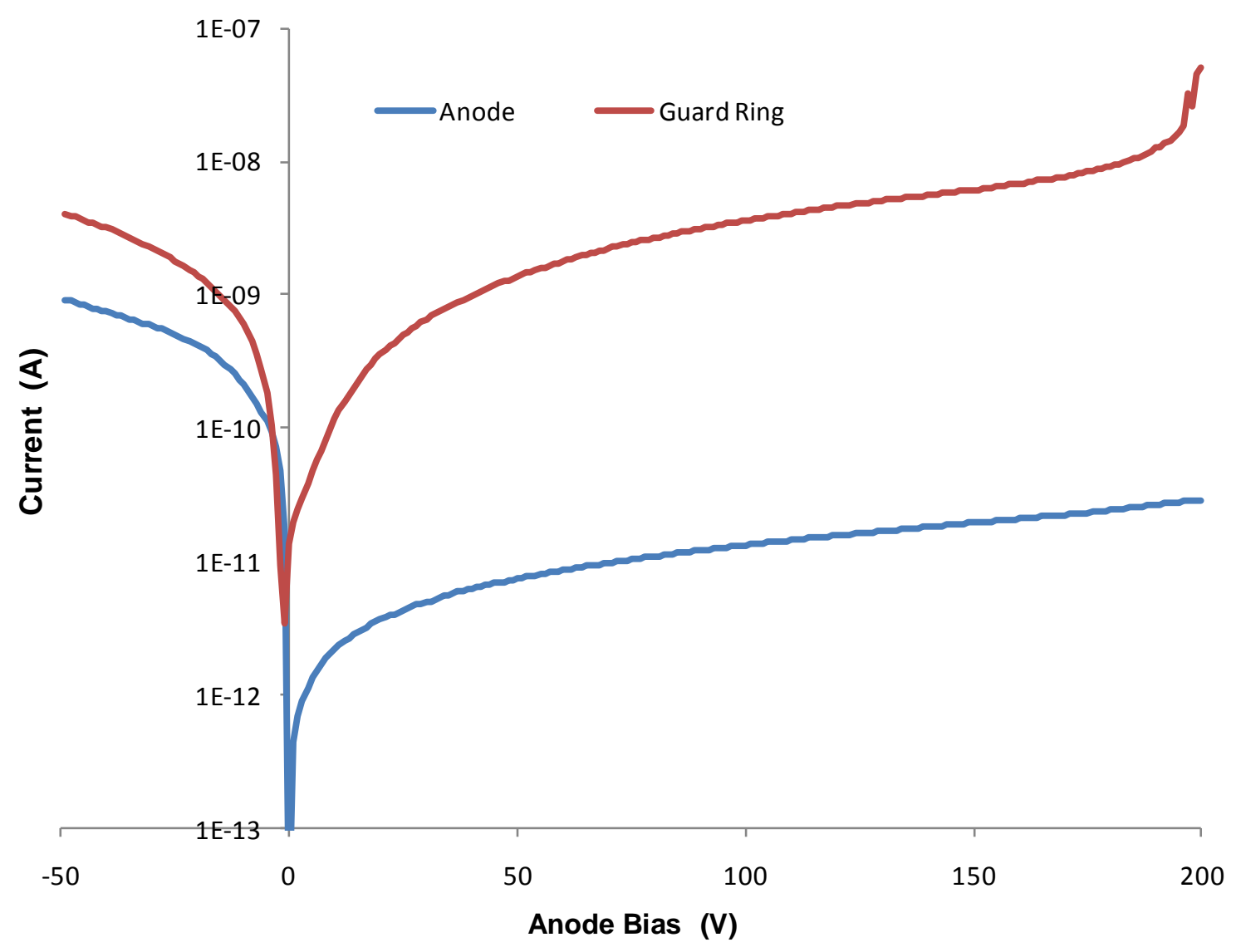

Figure 4.3 Anode and Guard Ring Currents at Room Temperature for a Typical Device

\subsection{Measurements}

Figure 4.4 shows the measurement system used for the current versus voltage measurements. The hot chuck is used for higher temperature measurements; this data is useful in differentiating between current mechanisms that have the same reverse bias voltage dependence. 


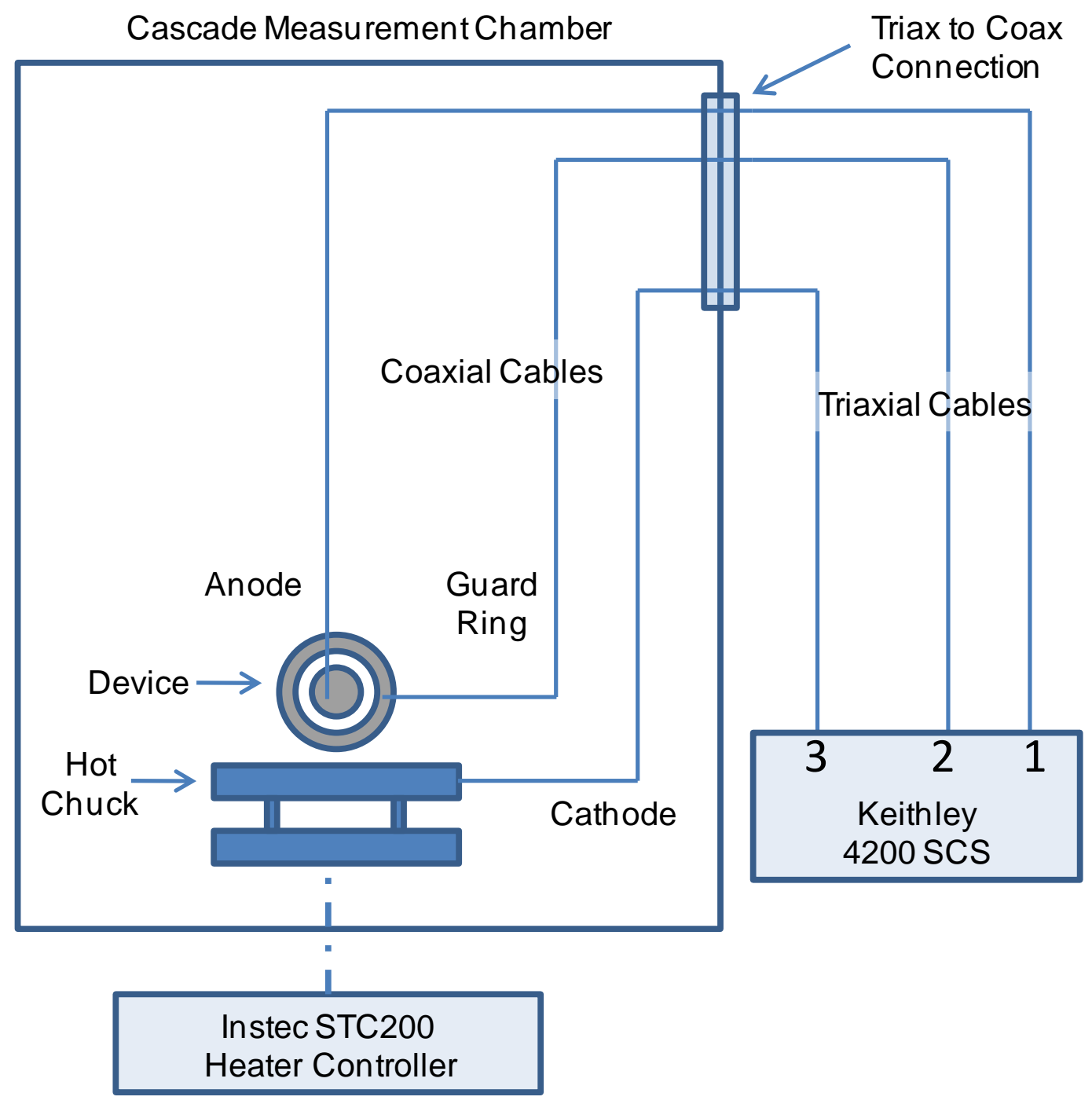

Figure 4.4 Current-Voltage Measurement System

Triaxial cables (triax) contain a guard around the center conductor that follows the bias on the center. With no potential difference between the center and this guard, there is no leakage through the dielectric; this guard also shields signals carried by the center conductor from RF interference. There is still leakage between the guard and the shield (ground), but this is unimportant since only the center conductor contacts the device under test [28], [29]. However, the triax is only present between the Keithley 4200 SCS and the Cascade measurement chamber. At that point a triax to coax connector leaves the 
biased guard open, connecting only the triax ground and center conductor to the coaxial cables.

\subsubsection{Keithley 4200 Semiconductor Characterization System}

The Keithley 4200 Semiconductor Characterization System (SCS) performs all current-voltage measurements. The probe used to measure the anode has an additional preamp that extends the measurement range by five orders of magnitude. Measurements are configured through a GUI interface on a windows-based platform. The automated measurements have user-defined delay, filtering, and integration settings. The delay holds a bias for a set amount of time before taking measurements, to reduce any transients. The filtering and $\mathrm{A} / \mathrm{D}$ integration average multiple measurements to reduce measurement noise. All of these settings scale as the current range changes, in order to optimize measurement speed and accuracy. The system can supply up to $210 \mathrm{~V}$ and can measure down to the $1 \mathrm{pA}$ range with $0.1 \mathrm{fA}$ resolution, both of which are important for the high resistivity CdTe detectors.

\subsubsection{Device Measurements}

Measurements are taken over separate low and high voltage ranges. The low voltage range is from $-2 \mathrm{~V}$ to $+2 \mathrm{~V}$, with the bias applied to the anode. This range is a standard in this field for detector comparison and is used for device parameter extraction. The high voltage range is from $50 \mathrm{~V}$ forward bias to $200 \mathrm{~V}$ reverse bias; the bias is applied to the cathode, since the preamp probe on the anode cannot supply more than $40 \mathrm{~V}$. High reverse bias is where the device will operate during radiation measurements, it gives additional information about current mechanisms in the device, 
and it shows whether or not the device enters breakdown. Figure 4.5 shows these two configurations.

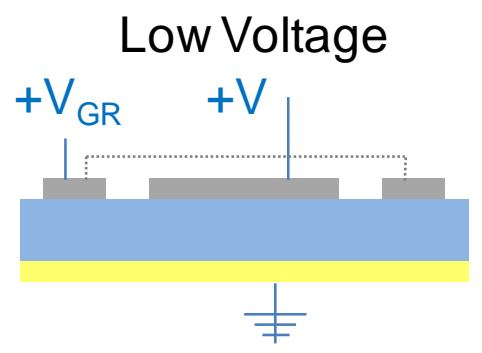

High Voltage

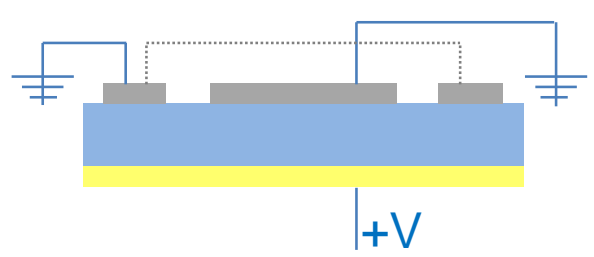

Figure 4.5 Device I-V Measurement Configuration

\subsubsection{Measurement Error Sources}

There are three minor sources of error and one major source: measurement equipment accuracy, cable dielectric leakage, cable capacitance, and coupling with the hot chuck. The Keithley 4200 SCS measurement error is $1 \%$ or less at all measurement ranges, as shown in table 4.2.

Table 4.2 Keithley 4200 SCS Measurement Accuracy

\begin{tabular}{|l|l|l|l|l|}
\hline Range & $1 \mathrm{pA}$ & $10 \mathrm{pA}$ & $100 \mathrm{pA}$ & $1 \mathrm{nA}$ \\
\hline Maximum Error & $1 \%$ & $0.5 \%$ & $0.1 \%$ & $0.05 \%$ \\
\hline
\end{tabular}

There are triaxial cables from the Keithley 4200 SCS to the measurement chamber, but at the wall of the chamber these are connected to coaxial cables; so the coaxial cables limit the performance of these connections. However, there is no measureable leakage current through the coax dielectric. Capacitive charging in the cables could be significant for high frequency measurements, but the detectors are measured at DC with the voltage stepped slowly. The noise from these sources is insignificant compared to that from the 
electrically-heated chuck: the chuck heats via a pulse width modulated source current that dissipates heat in the resistive chuck.

The I-V curve in figure 4.6 shows coupling between the device measurement and the chuck heating circuit. The first two attempts show significant errors, and the indicated noise in the third measurement still interferes with analytical modeling.

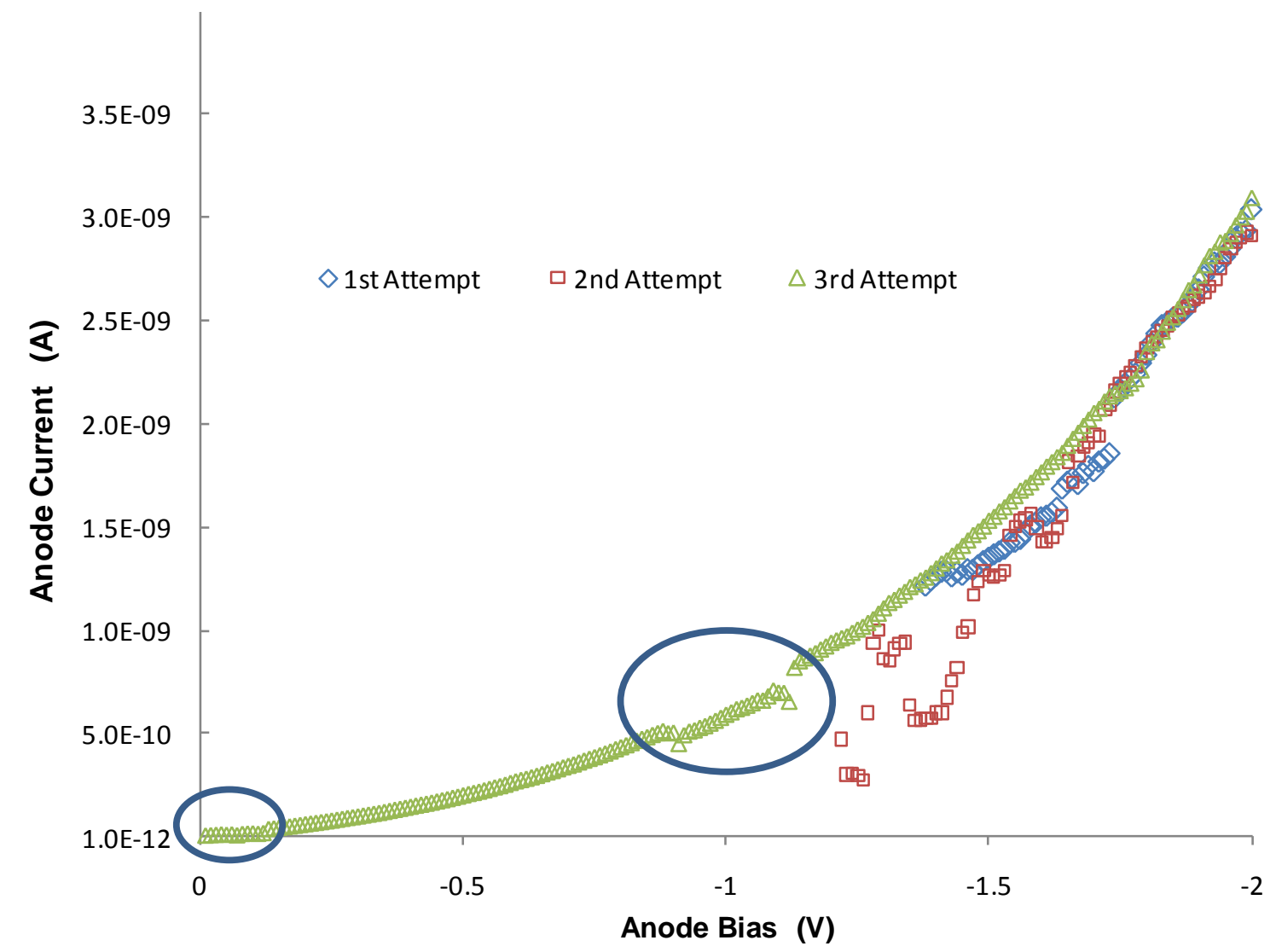

Figure 4.6 Measurement Error Caused by Hot Chuck at $70^{\circ} \mathrm{C}$

Using a triaxial chuck, rather than the present coaxial model, should significantly reduce coupling between the chuck heating circuit and the measurement circuit. The triaxial chuck is surrounded on the sides and bottom by a guard voltage that shields the 
measurement circuit from chuck heating cycles. Using triaxial probes and cables inside the chamber would also decrease coupling, since any noise currents would be induced in the guard, not the center conductor. By measuring over different ranges, changing the measurement speed, and re-calibrating the Keithley 4200 SCS, it is possible to obtain repeatable measurements with the current system configuration; these repeatable measurements accurately represent the device performance. 


\section{MEASUREMENT RESULTS AND ANALYSIS}

The devices are modeled based on the measurements using analytical current conduction models. Schottky diodes modeled by dominant thermionic emission (TE) current are common in literature [30], [31]. Modeling the detectors as single Schottky diodes dominated by thermionic emission provides a first-order estimation of device parameters; these parameters can be extracted using a single current-voltage characteristic or current-voltage data over a range of temperatures; each method has advantages and limitations.

\subsection{Thermionic Emission}

Thermionic emission involves thermal excitation of a carrier over a potential barrier. Figure 5.1 shows the thermionic emission processes in forward and reverse bias for a Schottky contact on p-type material; this section assumes dominant hole conduction for the p-type CdTe, as shown in figure 5.1. 


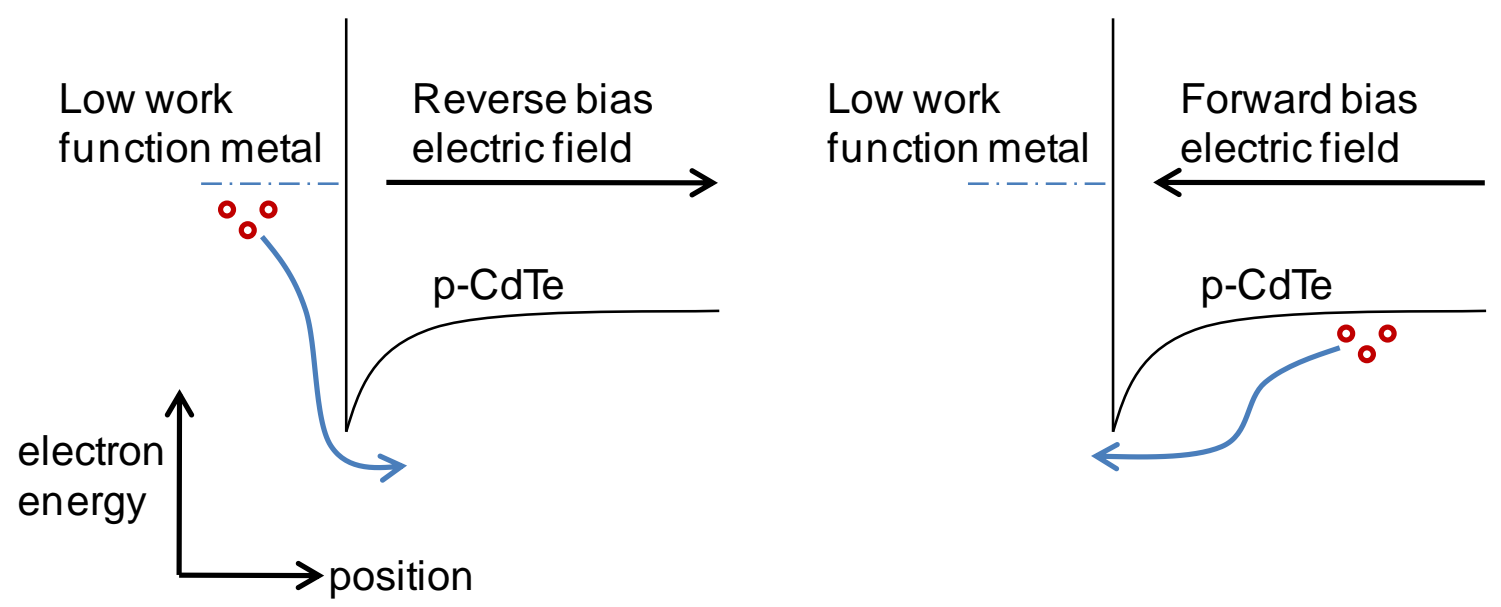

Figure 5.1 Thermionic Emission Processes

Equations 5.1 and 5.2 describe the current-voltage relationship for thermionic emission; the exponential component of equation 5.1 represents conduction from semiconductor to metal, and the ' -1 ' accounts for metal to semiconductor current.

$$
\begin{gathered}
I=I_{S}\left[\exp \left(\frac{q V}{n k T}\right)-1\right] \\
I_{S}=A A^{*} T^{2} \exp \left(\frac{-q \phi_{B}}{k T}\right)
\end{gathered}
$$

$\mathrm{I}_{\mathrm{S}}$ is the TE saturation current (amps), $\mathrm{q}$ is the electron charge (coulombs), $\mathrm{V}$ is the applied bias voltage (volts), $\mathrm{n}$ is the diode ideality factor, $\mathrm{k}$ is Boltzmann's constant $(\mathrm{J} / \mathrm{K})$, $\mathrm{T}$ is temperature $(\mathrm{K}), \mathrm{A}$ is the effective device area $\left(\mathrm{cm}^{2}\right), \mathrm{A}^{*}$ is the Richardson's constant $\left(\mathrm{A} / \mathrm{cm}^{2} \cdot \mathrm{K}^{2}\right)$, and $\phi_{\mathrm{B}}$ is the effective Schottky barrier height $(\mathrm{eV})$. The effective area used in calculations is that of the anode contact; this is not exactly the functional device area, but the error introduced by this assumption is minor compared to other sources. The linear plot in figure 5.2 shows a typical I-V characteristic at room temperature for the no sputter etch device; all I-V curves in this chapter come from measurements of the no 
sputter etch device unless otherwise specified. Recall from section 3.2 that the diode polarity is due to the built in potential from anode to cathode.

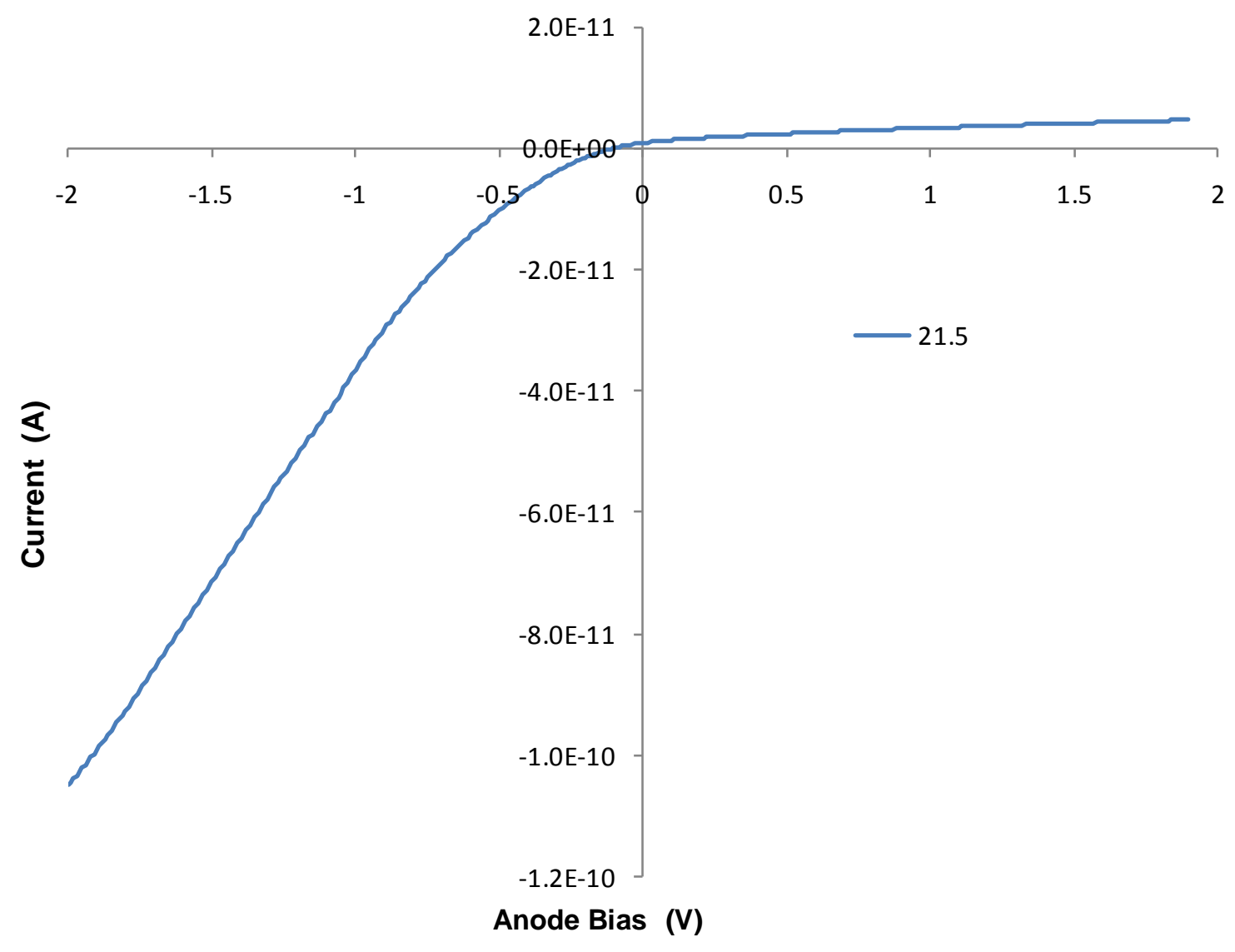

Figure 5.2 Typical I-V at Room Temperature for the No Sputter Etch Device

Figure 5.3 shows initial current-voltage measurements with strong temperature dependence, supporting the TE model shown in equations 5.1 and 5.2. 


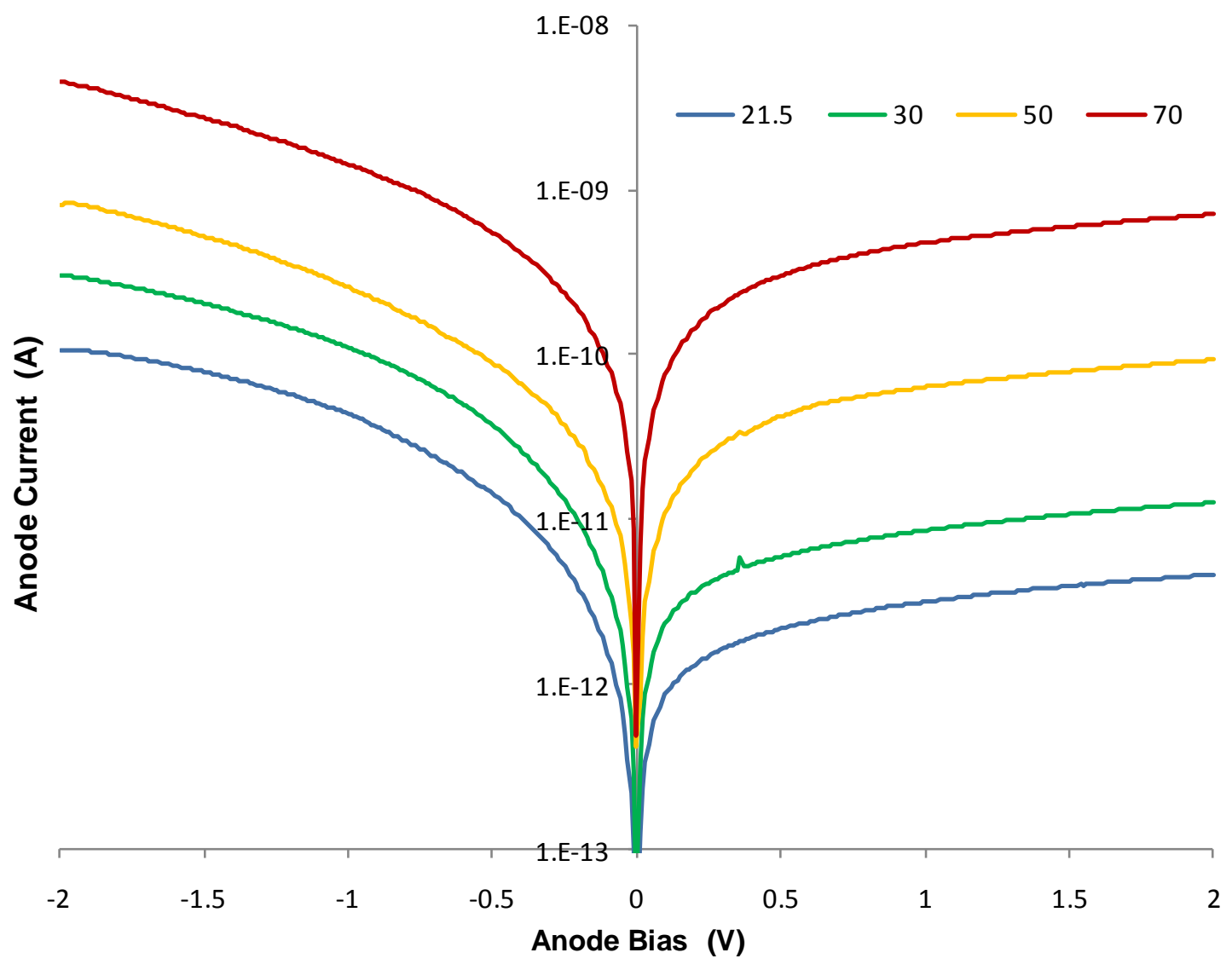

Figure 5.3 Typical I-V for the No Sputter Etch Device

\subsubsection{Current versus Voltage Extraction Method}

The ideality factor $(\mathrm{n})$, saturation current $\left(\mathrm{I}_{\mathrm{S}}\right)$, and barrier height $\left(\phi_{\mathrm{B}}\right)$ can be determined from the forward bias data of a single I-V curve. Recall from section 4.2.2 that forward bias refers to negative voltage applied to the anode. On a semilog plot, there is a linear portion of the I-V curve at low voltage; this linear portion can be extrapolated to determine the $y$-intercept, equal to $\mathrm{I}_{\mathrm{S}}$, and the slope, inversely proportional to ideality factor, $n$ : 


$$
\begin{gathered}
I_{S}=e^{b} \\
n=\left[\frac{k T}{q} \frac{d(\ln I)}{d V}\right]^{-1}
\end{gathered}
$$

where $b$ is the $y$-intercept of the linear extrapolation. The ideality factor should be close to $\mathrm{n}=1$ for perfect TE, but it will approach $\mathrm{n}=2$ as the contribution from generationrecombination current increases [17]. As other current mechanisms become significant, the ideality factor can also exceed $n=2$, with values as high as $n=8.9$ reported [32]; these high ideality factors are sometimes attributed to a native oxide layer on the electrode and an inhomogeneous barrier [32]. With an ideality higher than $\mathrm{n} \approx 1.1$, contributions from other sources are too significant to calculate $\mathrm{I}_{\mathrm{S}}$ for $\mathrm{TE}$ alone; the barrier height calculated from $\mathrm{I}_{\mathrm{S}}$ will then have no physical interpretation [33].

Equation 5.5 yields the effective barrier height:

$$
\phi_{B}=\frac{k T}{q} \ln \left(\frac{A A^{*} T^{2}}{I_{S}}\right)
$$

where $I_{S}$ comes from prior extraction and the Richardson's constant is $A^{*} \approx 48 \mathrm{~A} / \mathrm{cm}^{2} \cdot \mathrm{K}^{2}$ for p-CdTe [20]. However, the Richardson's constant depends on surface preparation, metal deposition technique, contact thickness, and contact metal [34], [35]. Therefore, the Richardson's constant obtained from the literature may not be correct for our device, and the barrier height calculated from this technique is only as accurate as our knowledge of this parameter [36].

As the forward bias voltage increases, more potential drops across the undepleted bulk region, and the potential across the anode metal-semiconductor junction changes very little. On a semilog plot, this yields a non-linear I-V characteristic, limiting the 
linear range available for parameter extraction. Figure 5.4 shows the device structure and the equivalent circuit.

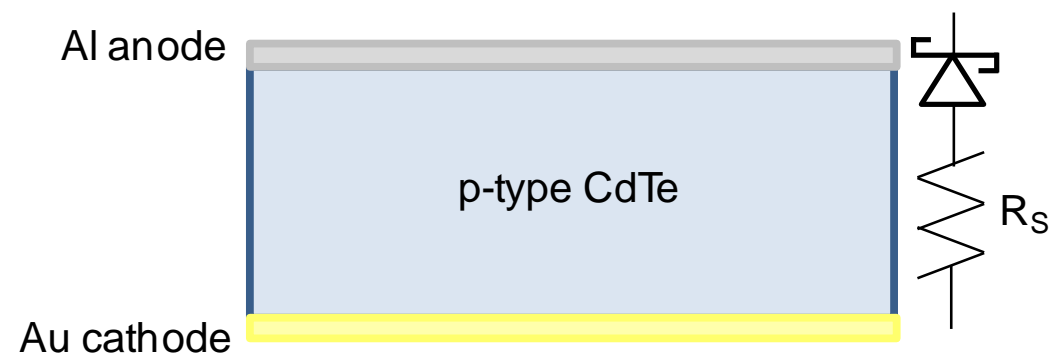

Figure 5.4 Device Structure and Equivalent Circuit

An approximate series resistance, $R_{S}$, can be determined from the inverse slope of the linear I-V curve at higher voltages, where the diode has sufficiently small differential resistance:

$$
R_{S}=\left[\frac{d(\ln I)}{d V}\right]^{-1}
$$

Other series resistance extraction methods that account for diode differential resistance yielded similar results [37]. By modifying TE theory to account for series resistance, the plot can be partially linearized for more reliable parameter extraction; this also decreases ideality factor making the thermionic emission model more accurate. Equation 5.7 shows the TE model corrected for the voltage drop across a series resistance.

$$
I=I_{S}\left[\exp \left(\frac{q\left(V-I R_{S}\right)}{n k T}\right)-1\right]
$$

Figure 5.5 shows the I-V characteristic at room temperature with and without accounting for series resistance. The measured data assumes $V_{\text {DIODE }}=V_{\text {APPLIED, }}$, whereas for the curve corrected for series resistance $\mathrm{V}_{\text {DIODE }}=\mathrm{V}_{\mathrm{APPLIED}}-\mathrm{I} \cdot \mathrm{R}_{\mathrm{S}}$. 


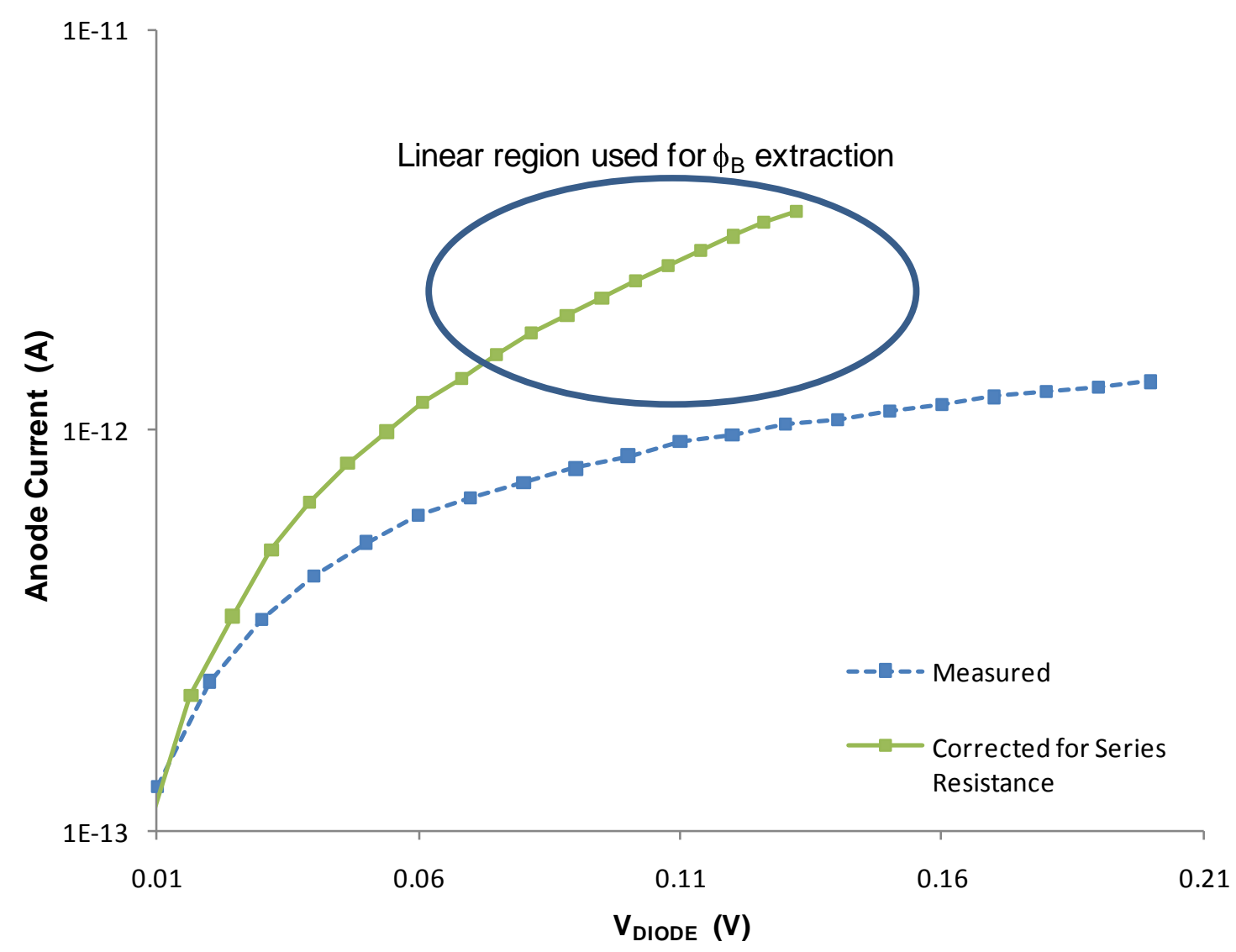

Figure 5.5 I-V Curve at Room Temperature With and Without Correcting For Series Resistance

Only the linear region above $V \gg k T / q$ can be used for parameter extraction, since the extraction equations ignore metal to semiconductor current (the ' -1 ' in equation 5.7); this limits the amount of useful data and increases statistical error. The current-voltage extraction curve can be linearized at low voltages by modifying the improved thermionic emission model in equation 5.7 to include the non-ideality in metal to semiconductor thermionic emission. To account for metal to semiconductor nonideality, we must assume that the barrier height changes due to image force barrier lowering and other effects associated with the metal-semiconductor junction [38]. If the barrier height varies linearly with forward bias voltage and ideality factor is defined in terms of barrier height, equation 5.7 can be rewritten as equation 5.8. 


$$
I=I_{S} \exp \left(\frac{q\left(V-I R_{S}\right)}{n k T}\right)\left[1-\exp \left(-\frac{q\left(V-I R_{S}\right)}{k T}\right)\right]
$$

Plotting equation 5.8 with $\left\{\ln (I) /\left[1-\exp \left(-\frac{q\left(V-I R_{S}\right)}{k T}\right)\right]\right\}$ on the y-axis yields an extraction plot with valid data down to $0 \mathrm{~V}$, maximizing the useful data range [36]. The I-V extraction curve in figure 5.6 accounts for series resistance and non-ideality in the metal-semiconductor current. The curve is still non-linear indicating that the TE model alone may not be sufficient for this device.

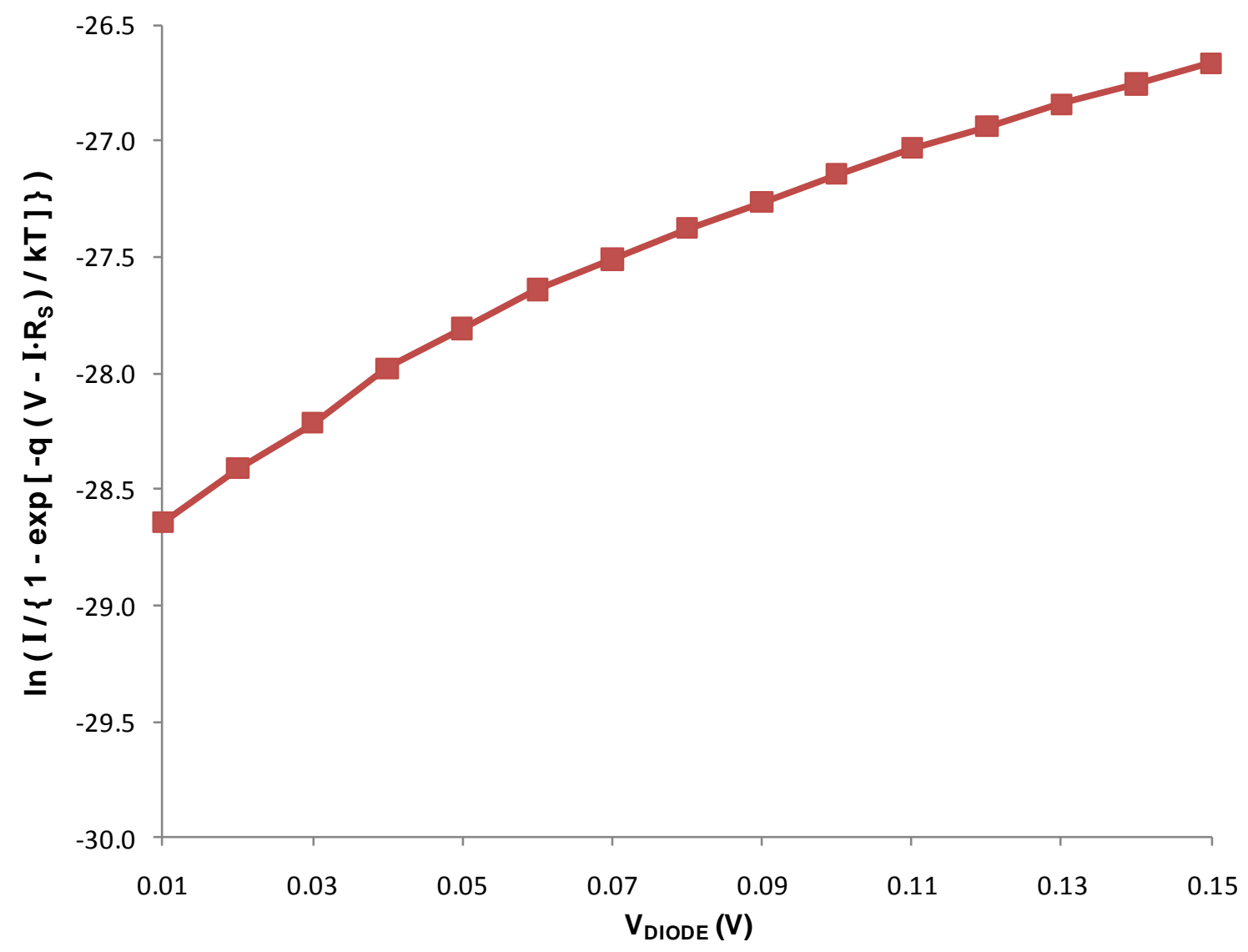

Figure 5.6 I-V Parameter Extraction Plot Corrected For $\mathbf{R}_{S}$ and Metal-Semiconductor Non-Ideality

\subsubsection{Current versus Temperature Extraction Method}

This method uses current-voltage data at multiple temperatures, yielding values for barrier height, $\phi_{\mathrm{B}}$, and Richardson's constant, $\mathrm{A}^{*}$. Due to the discrete nature of the 
measured data, it is very difficult to correct for series resistance and metal-semiconductor non-ideality in this method. By assuming $V \gg k T / q$, equation 5.1 is rewritten as equation 5.9.

$$
\ln \left(\frac{I}{T^{2}}\right)=\ln \left(A A^{*}\right)-\frac{q}{k T}\left(\phi_{B}-V / n\right)
$$

On an Arrhenius plot with axes $I / T^{2}$ versus $1000 / T$, barrier height is related to the slope of the curve, and the Richardson's constant is derived from the y-intercept; figure 5.7 shows an Arrhenius plot for the no sputter etch device.

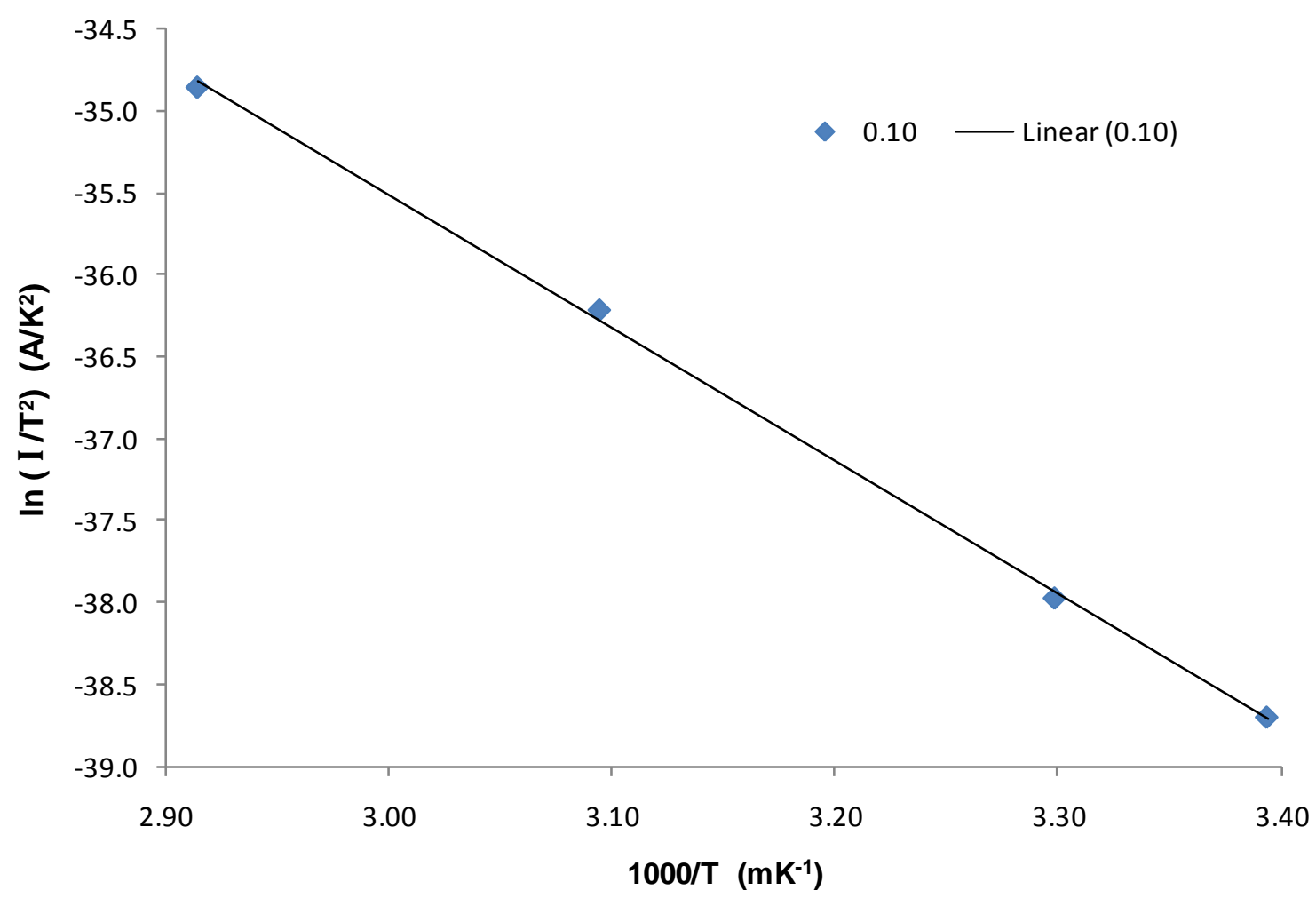

Figure 5.7 Arrhenius Plot for Current-Temperature Parameter Extraction

Equations 5.10 and 5.11 show the equations used to extract barrier height and Richardson's constant, where b is the y-intercept of the least-squares fit in figure 5.7. However, the small temperature measurement range, $21.5^{\circ} \mathrm{C}$ (room temperature) to 
$70{ }^{\circ} \mathrm{C}$, introduces enormous error in this extracted Richardson's constant, since it is derived from the y-intercept at infinitely high temperature.

$$
\begin{gathered}
\phi_{B}=\frac{V}{n}-\frac{k}{q} \frac{d\left[\ln \left(I / T^{2}\right)\right]}{d[1 / T]} \\
A^{*}=\frac{1}{A} e^{b}
\end{gathered}
$$

The current-voltage (I-V) method and current-temperature (I-V-T) methods are coupled: the barrier height extraction in the I-V-T method depends on ideality factor from the I-V method, and the extracted Richardson's constant from the I-V-T method can be used in calculating the barrier height in the I-V method.

\subsubsection{Extraction Results}

Table 5.1 compares the accuracy of I-V and I-V-T extraction results for a $500 \mu \mathrm{m}$ diameter no sputter etch device; the I-V extraction uses room temperature data.

Table 5.1 Extracted Values for $500 \mu \mathrm{m}$ Diameter No Sputter Etch Device

\begin{tabular}{|l|c|c|}
\hline & Barrier height, $\phi_{\mathrm{B}}(\mathrm{eV})$ & Ideality factor, $\mathrm{n}$ \\
\hline $\mathrm{I}-\mathrm{V}$ & 0.70 & 3.4 \\
\hline $\mathrm{I}-\mathrm{V}$ corrected for series resistance, $\mathrm{R}_{\mathrm{S}}$ & 0.70 & 2.3 \\
\hline $\begin{array}{l}\text { I-V corrected for } \mathrm{R}_{\mathrm{S}} \text { and } \\
\text { metal-semiconductor non-ideality }\end{array}$ & 0.71 & 1.5 \\
\hline $\mathrm{I}-\mathrm{V}-\mathrm{T}$ & 0.71 & - \\
\hline
\end{tabular}

The various improvements on the I-V method only slightly affect the extracted barrier height, but have a very significant effect on the ideality factor. The changes in ideality factor indicate that each modification of the I-V extraction method yields a more accurate model for the measured devices. The I-V and I-V-T methods agree well because the 
Richardson's constant extracted with I-V-T is used to calculate the barrier height in the I-V method.

Figures 5.8 shows the measured low voltage current-voltage characteristics for the $500 \mu \mathrm{m}$ diameter no sputter etch and sputter etch devices. At room temperature, the sputter etch device has $50 \%$ less current than the no sputter etch device at $2 \mathrm{~V}$ reverse bias.
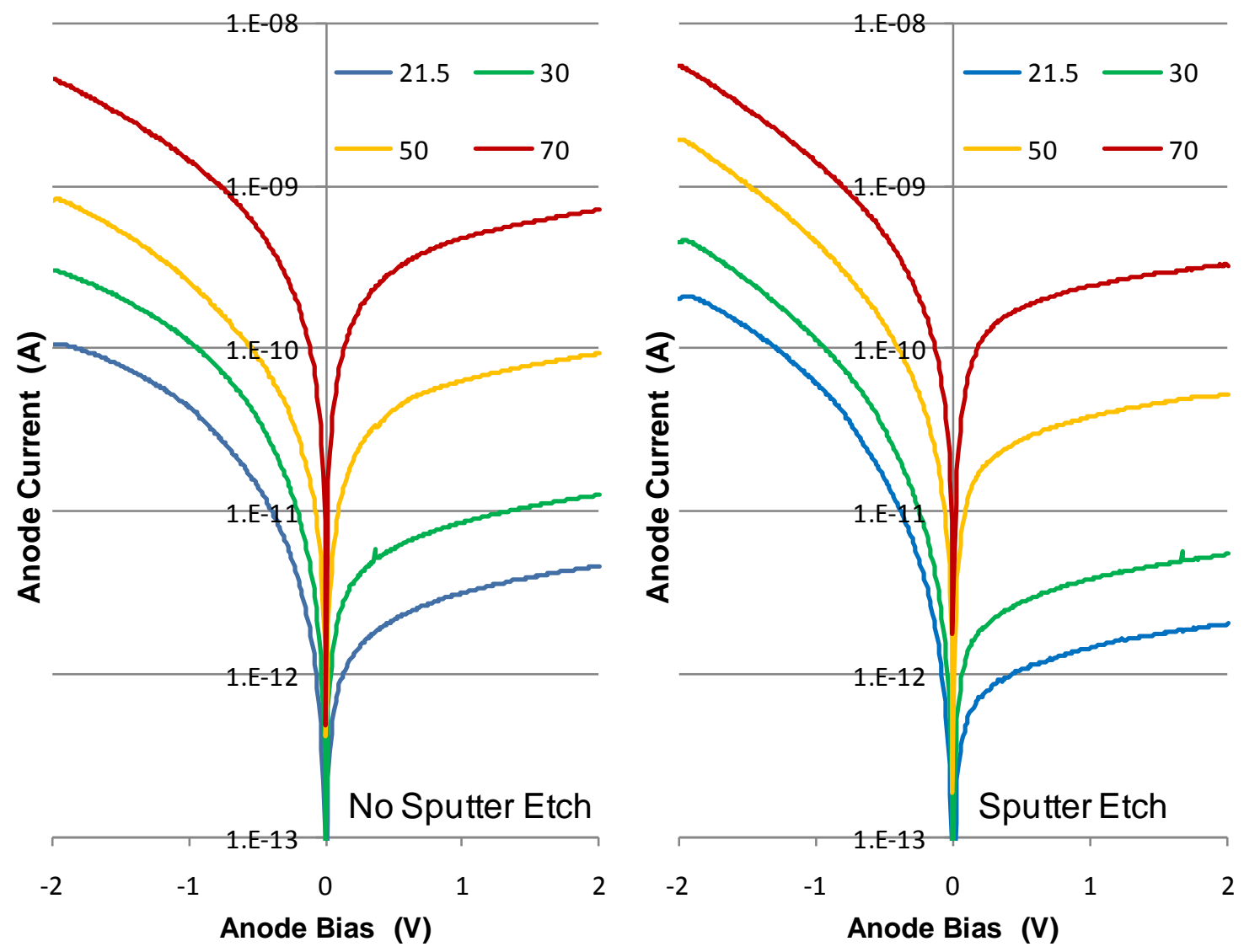

Figure 5.8 Measured Low Voltage Current-Voltage Characteristic for $500 \mu \mathrm{m}$ Devices

Table 5.2 summarizes the $500 \mu \mathrm{m}$ device parameters extracted using the I-V-T method and the most accurate I-V method, and table 5.3 summarizes the $250 \mu \mathrm{m}$ device parameters. The ideality factor is extracted using room temperature current-voltage data. 
Table 5.2 Extracted $500 \mu \mathrm{m}$ Device Parameters at Room Temperature

\begin{tabular}{|c|c|c|c|c|}
\hline & $\begin{array}{c}\text { Barrier height, } \\
\phi_{\mathrm{B}}(\mathrm{eV})\end{array}$ & $\begin{array}{c}\text { Ideality } \\
\text { factor, } \mathrm{n}\end{array}$ & $\begin{array}{c}\text { Richardson's constant, } \\
\mathrm{A}^{*}\left(\mathrm{~A} / \mathrm{cm}^{2} \cdot \mathrm{K}^{2}\right)\end{array}$ & $\begin{array}{c}\text { Effective resistivity at } \\
2 \mathrm{~V} \text { reverse bias }(\Omega \cdot \mathrm{cm})\end{array}$ \\
\hline $\begin{array}{c}\text { No sputter } \\
\text { etch }\end{array}$ & 0.71 & 1.5 & $2 \times 10^{-3}$ & $8.4 \times 10^{9}$ \\
\hline Sputter etch & 0.75 & 1.8 & $8 \times 10^{-3}$ & $1.9 \times 10^{10}$ \\
\hline
\end{tabular}

Table 5.3 Extracted 250 $\mu$ m Device Parameters at Room Temperature

\begin{tabular}{|c|c|c|c|c|}
\hline & $\begin{array}{c}\text { Barrier height, } \\
\phi_{\mathrm{B}}(\mathrm{eV})\end{array}$ & $\begin{array}{c}\text { Ideality } \\
\text { factor, } \mathrm{n}\end{array}$ & $\begin{array}{c}\text { Richardson's constant, } \\
\mathrm{A}^{*}\left(\mathrm{~A} / \mathrm{cm}^{2} \cdot \mathrm{K}^{2}\right)\end{array}$ & $\begin{array}{c}\text { Effective resistivity at } \\
2 \mathrm{~V} \text { reverse bias }(\Omega \cdot \mathrm{cm})\end{array}$ \\
\hline $\begin{array}{c}\text { No sputter } \\
\text { etch }\end{array}$ & 0.74 & 1.3 & $9 \times 10^{-3}$ & $6.6 \times 10^{9}$ \\
\hline Sputter etch & 0.78 & 1.7 & $4 \times 10^{-2}$ & $1.8 \times 10^{10}$ \\
\hline
\end{tabular}

The effective resistivity is a performance metric independent of the conduction model:

$$
\rho_{\text {eff }}=\frac{V_{r}}{J L}
$$

where $\mathrm{V}_{\mathrm{r}}$ is the reverse bias voltage, $\mathrm{J}$ is the anode current density, and $\mathrm{L}$ is the device thickness. The close agreement between the effective resistivity in the $500 \mu \mathrm{m}$ and $250 \mu \mathrm{m}$ diameter devices indicates that the anode area is an appropriate value to use as the effective device area.

The higher extracted barrier heights in the sputter etch devices correlate with the higher effective resistivity, but the ideality factor indicates that a simple thermionic emission model does not accurately represent these devices; and with such high ideality factors, the extracted barrier height is not physically meaningful [33]. The low extracted Richardson's constant is much less than the $\mathrm{A}^{*} \approx 48 \mathrm{~A} / \mathrm{cm}^{2} \cdot \mathrm{K}^{2}$ predicted by theory:

$$
A^{*}=\frac{4 \pi q m^{*} k^{2}}{h^{3}}
$$

where $\mathrm{m}^{*}$ is the carrier effective mass and $\mathrm{h}$ is Planck's constant. The low extracted value is a combination of two factors: the extraction is inaccurate due to the long extrapolation 
to $\mathrm{T}=\infty$ from the data in figure 5.7 , and the Richardson's constant depends strongly on surface preparation, contact thickness, and contact metal [34]. The difference in parameters for different size devices, the extracted ideality factor, and the diode differential resistance suggest possible models to describe these devices.

Tables 5.2 and 5.3 indicate that the smaller devices have larger barriers, lower ideality factors, and Richardson's constants closer to theoretical values; an inaccurate effective area could not explain all of these, since ideality factor does not depend on area. One explanation for these parameter differences is an inhomogeneous Schottky barrier, where the barrier parameters vary across the contact area due to changes in surface characteristics, as shown in figure 5.9.

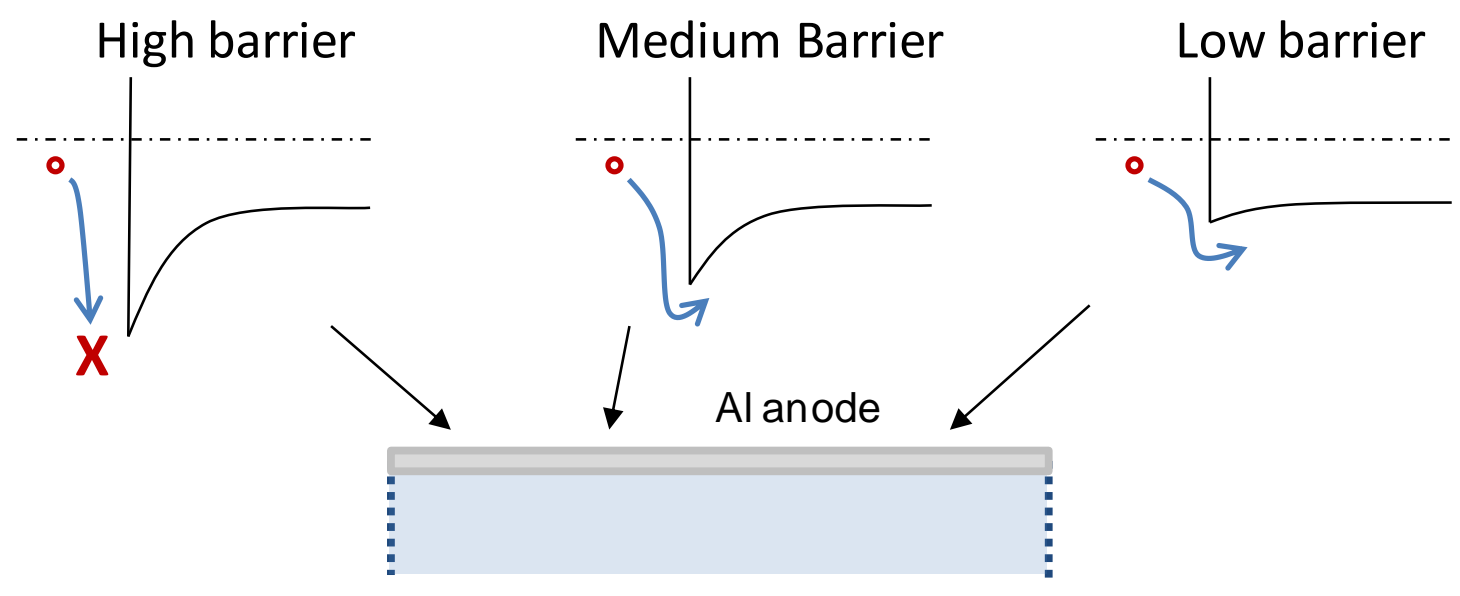

Figure 5.9 Inhomogeneous Schottky Barrier

A smaller anode contact should have less variation in surface characteristics across its area, yielding a more homogeneous barrier; a lower standard deviation in the actual barrier height across the contact yields a higher effective barrier height and lower effective ideality factor [37]; we can also postulate that a more homogeneous barrier 
yields a Richardson's constant closer to theoretical values. The measured data agrees with all of these conclusions.

Figure 5.10 shows the temperature dependence of the extracted ideality factors for these devices.

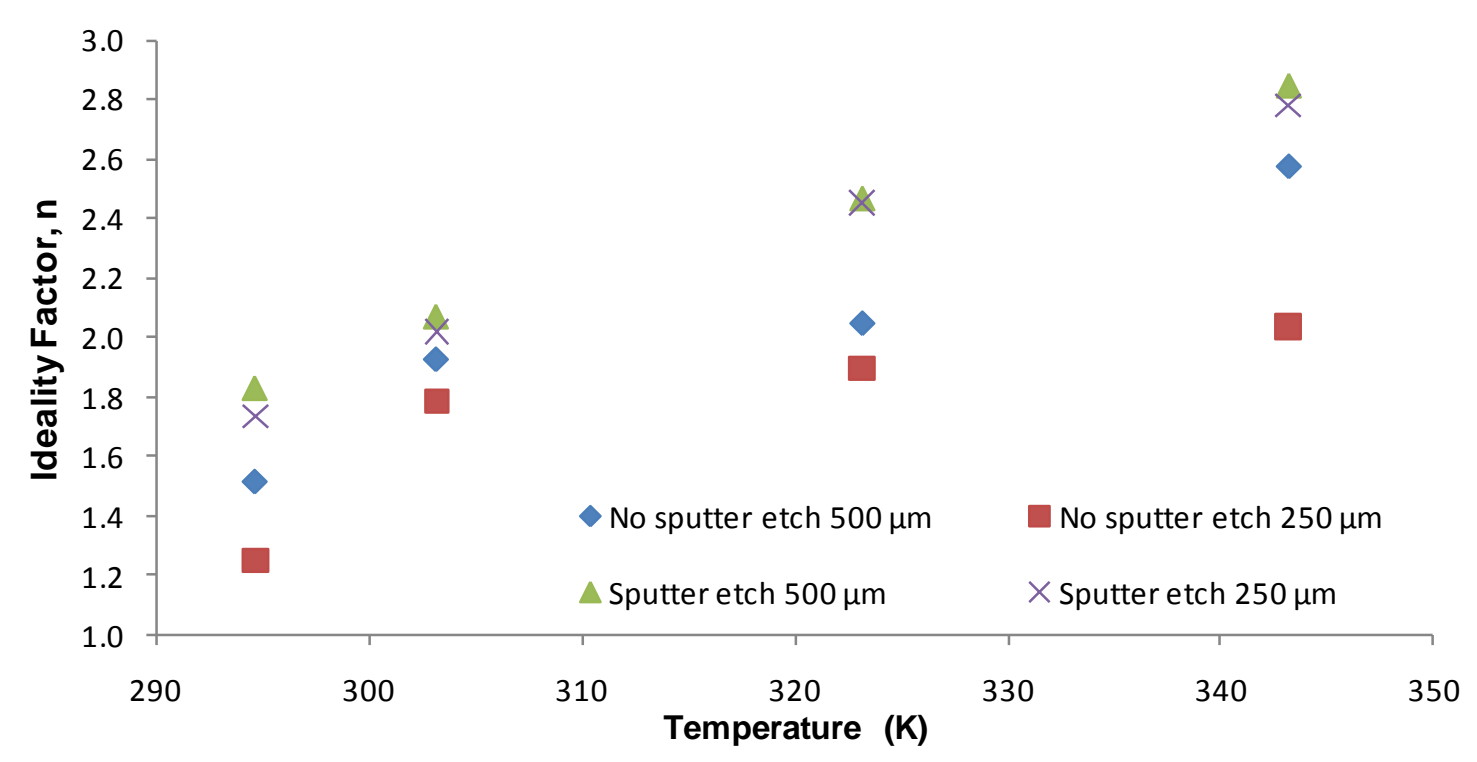

Figure 5.10 Ideality Factor Temperature Dependence

In thermionic emission theory, the ideality factor describes the voltage dependence of the barrier height [38], and that is independent of temperature. Increased generationrecombination current at elevated temperature and an inhomogeneous barrier can account for the ideality factor temperature dependence [37].

Figure 5.11 shows the current-voltage characteristics for the $500 \mu \mathrm{m}$ diameter no sputter etch and sputter etch devices at high voltage. 

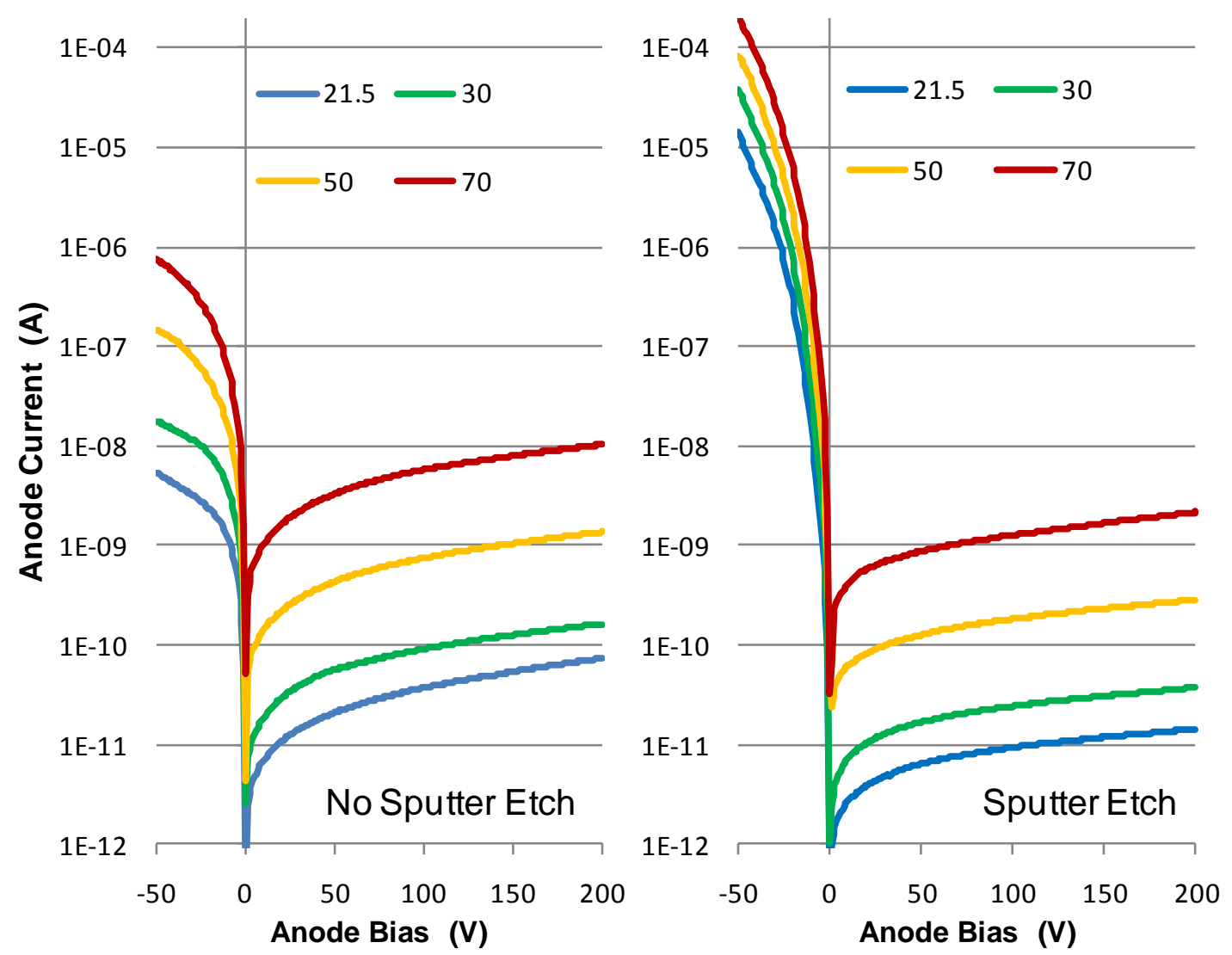

Figure 5.11 Measured High Voltage Current-Voltage Characteristic for $500 \mu \mathrm{m}$ Devices

In figure 5.11, the reverse bias characteristic is similar to that observed at low voltages, but the sputter etch device shows much higher forward bias current. The sputter etch removed the native oxide before contact deposition, but there are likely other factors contributing to the increased forward bias current.

Figure 5.12 shows the differential resistance for the $500 \mu \mathrm{m}$ diameter devices. 

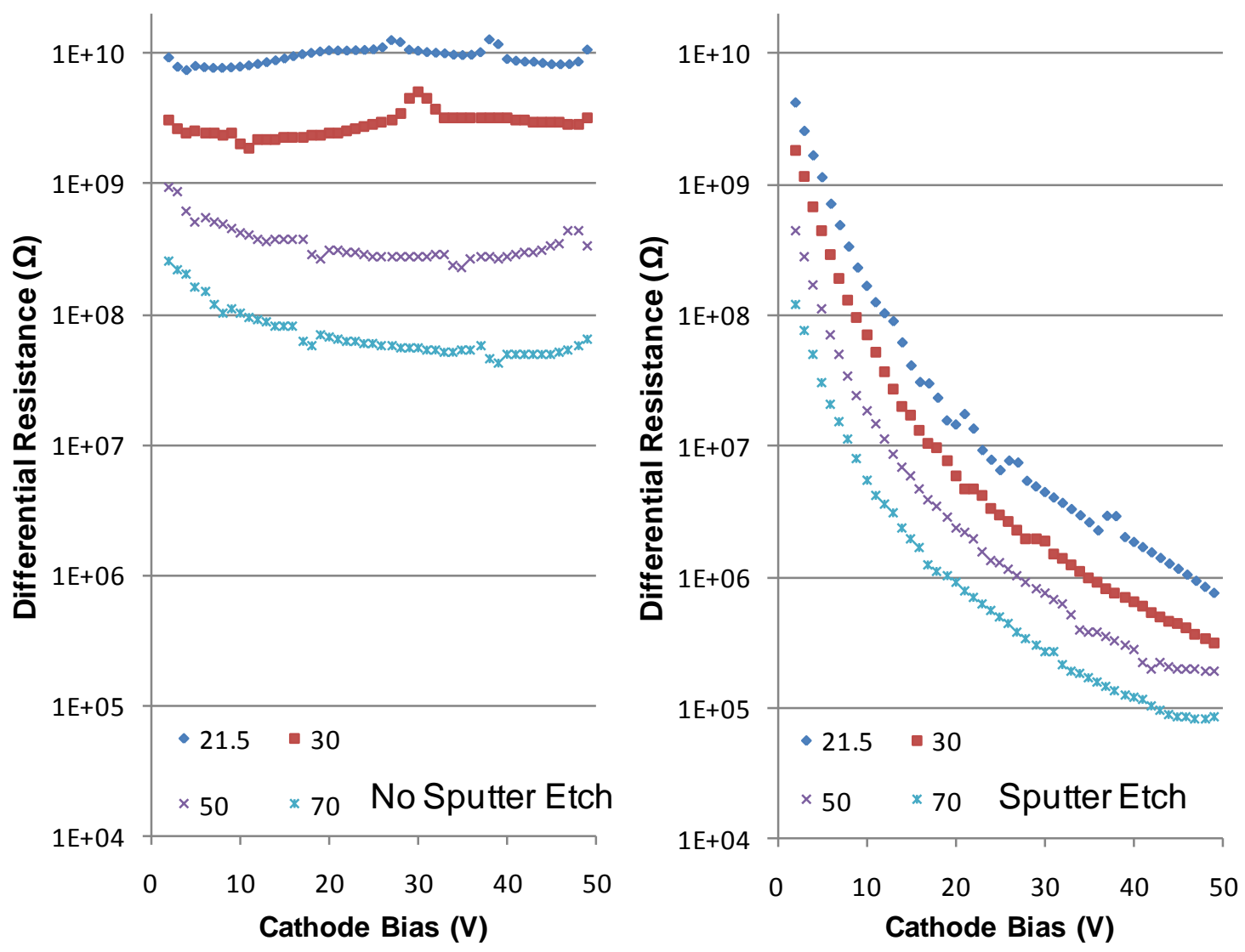

Figure 5.12 Differential Resistance for $500 \mu \mathrm{m}$ Devices

The differential resistance should decrease until the voltage across the diode ceases to change; the saturation value is the series resistance. This is what occurs in the no sputter etch device, but the differential resistance of the sputter etch device continues to decrease even at high voltages. The decreasing differential resistance can be explained by minority carrier injection from the anode [39]. The sputter etch changed the surface defect density and likely led to a higher anode hole barrier, and therefore a larger electron accumulation; electrons from this accumulation are injected from the anode further into the diode bulk with increasing applied bias, decreasing bulk resistance. This electron injection also contributes to the high ideality factor. The sputter etch may also have yielded a more homogeneous Schottky barrier, as previously suggested. 


\subsection{Leaky Schottky Barrier Model}

Lee et al reported a thermionic field emission model for a reverse-biased Schottky diode on high resistivity material [40]. The differential resistance for these devices suggests significant electron injection in forward bias, so modeling the devices in reverse bias may yield more accurate results. Equation 5.14 describes the reverse bias current in the thermionic field emission (TFE) model.

$$
I_{T F E}=\frac{A^{*} A T \sqrt{\pi q E_{00}}}{k}\left[q\left(V_{r}-I_{r} R_{S}-\frac{E_{G}}{2}+\phi_{B}\right)\right]^{1 / 2} \exp \left(-\frac{q \phi_{B}}{k T}\right)
$$

$\mathrm{E}_{\mathrm{G}}$ is the energy bandgap and $\mathrm{E}_{00}$ relates to carrier tunneling through the barrier:

$$
E_{00}=\frac{h}{4 \pi} \sqrt{\frac{N_{A}}{m^{*} \varepsilon}}
$$

where $\mathrm{N}_{\mathrm{A}}$ is total acceptor impurity concentration.

Applying this model to the no sputter etch device does not show sufficient current growth at high reverse bias. The temperature dependent ideality factor shown in the previous suggestion suggested an inhomogeneous barrier, so this model needs to be amended to account for leakage through the Schottky barrier; Donoval et al models this leakage as an ohmic shunt resistance as in figure 5.13 and equation 5.16 [33]:

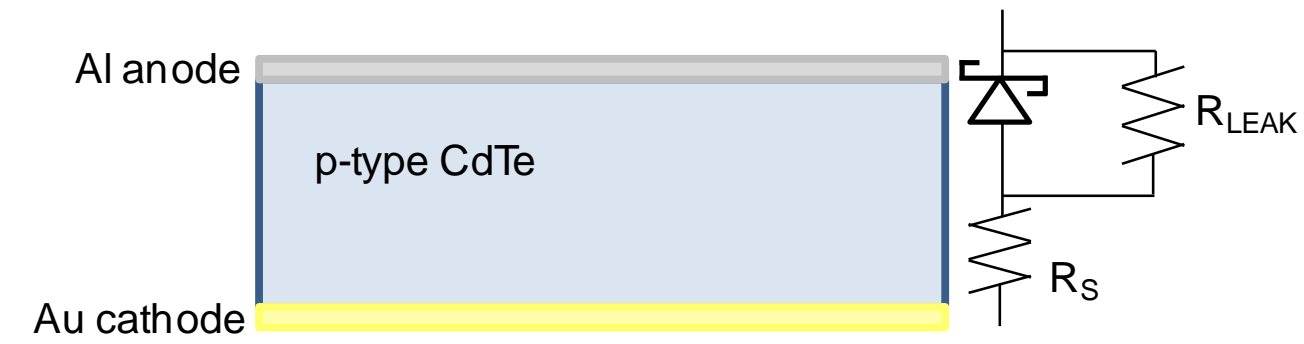

Figure 5.13 Device Structure and Equivalent Circuit with Shunt Resistance 


$$
I=I_{T F E}+\frac{V-I R_{S}}{R_{L E A K}}
$$

where $\mathrm{R}_{\mathrm{LEAK}}$ represents the ohmic leakage through the inhomogeneous barrier.

Figure 5.13 compares the measured data for the $500 \mu \mathrm{m}$ diameter no sputter etch device to this leaky Schottky barrier model; the sputter etch device had almost no ohmic component, as shown in figure 5.14. In both figures the measured data is a thin solid line and the model is represented by the thick, dashed line.

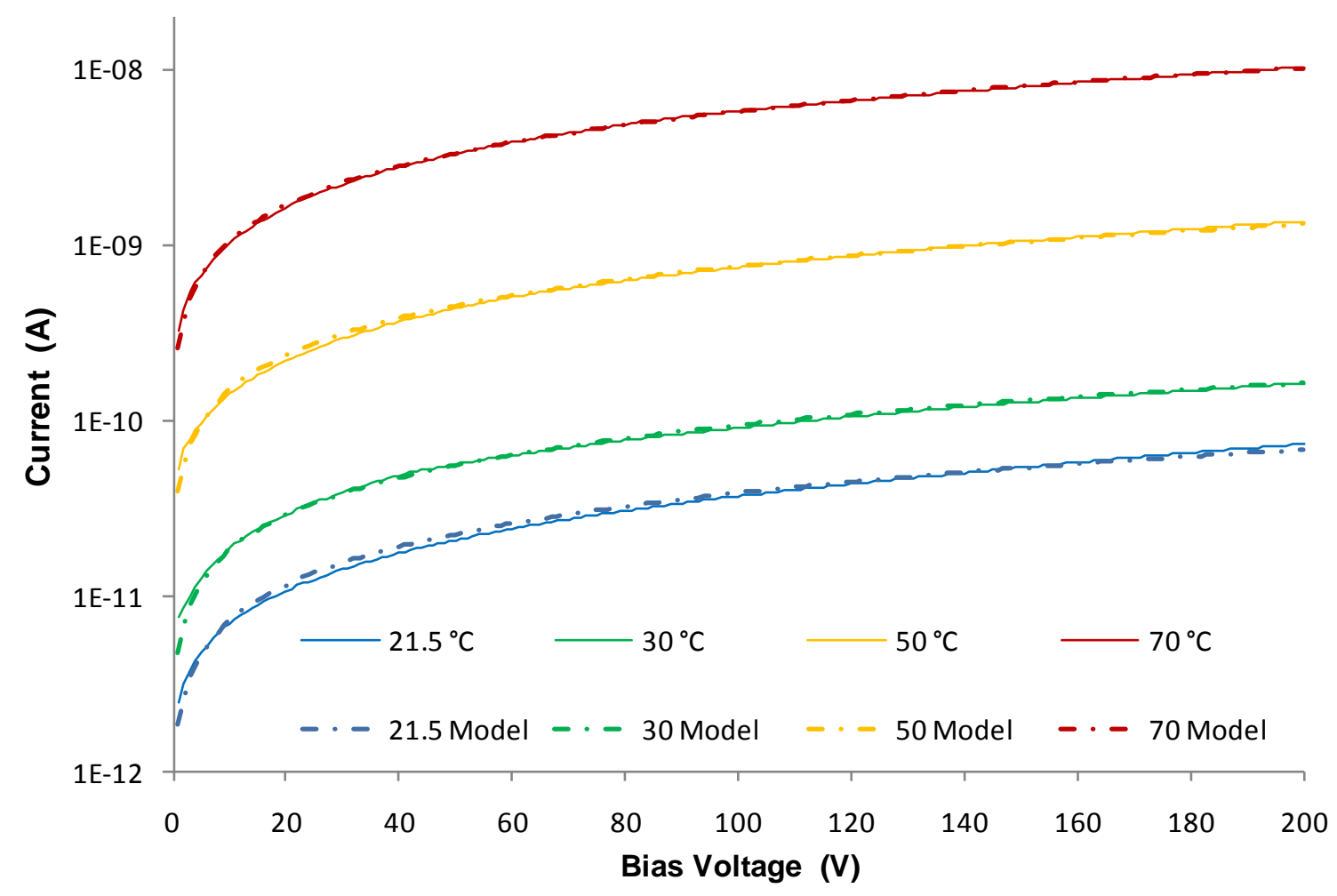

Figure 5.14 Leaky Schottky Barrier Model for $500 \mu \mathrm{m}$ Diameter No Sputter Etch Device 


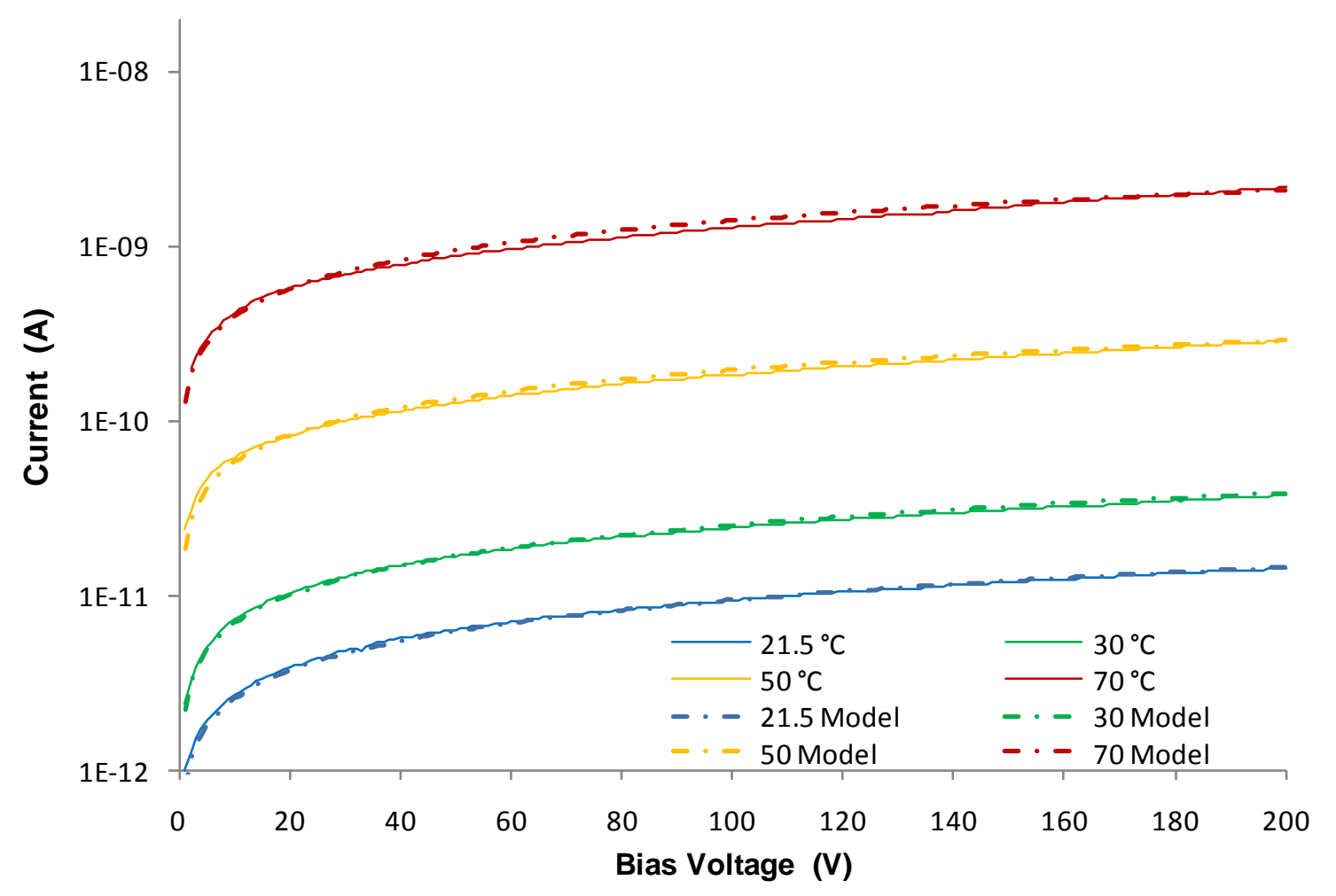

Figure 5.15 Leaky Schottky Barrier Model for $500 \mu \mathrm{m}$ Diameter Sputter Etch Device

Table 5.4 summarizes the leaky Schottky barrier model parameters for the $500 \mu \mathrm{m}$ diameter devices.

Table 5.4 Leaky Schottky Barrier Model Parameters for $500 \mu \mathrm{m}$ Devices

\begin{tabular}{|c|c|c|c|c|c|}
\hline & $\begin{array}{c}\text { Barrier } \\
\text { Height, } \phi_{\mathrm{B}}\end{array}$ & $\begin{array}{c}\text { Acceptor } \\
\text { Concentration, } \mathrm{N}_{\mathrm{A}}\end{array}$ & $\begin{array}{c}\text { Relative } \\
\text { Permittivity, } \varepsilon_{\mathrm{S}}\end{array}$ & $\begin{array}{c}\text { Bandgap, } \\
\mathrm{E}_{\mathrm{G}}\end{array}$ & $\begin{array}{c}\text { Hole effective } \\
\text { Mass, } \mathrm{m}^{*} / \mathrm{m}_{0}\end{array}$ \\
\hline $\begin{array}{c}\text { No Sputter } \\
\text { Etch }\end{array}$ & $0.865 \mathrm{eV}$ & $10^{11} \mathrm{~cm}^{-3}$ & 10 & $1.50 \mathrm{eV}$ & 0.4 \\
\hline $\begin{array}{c}\text { Sputter } \\
\text { Etch }\end{array}$ & $0.884 \mathrm{eV}$ & $10^{11} \mathrm{~cm}^{-3}$ & 10 & $1.50 \mathrm{eV}$ & 0.4 \\
\hline
\end{tabular}

The relatively low doping in both models yields a wide barrier and a small tunneling component, so the primary conduction mechanism in reverse bias is thermionic emission. The model is very sensitive to barrier height: a $1 \mathrm{meV}$ increase in barrier height 
noticeably decreases the current in the model. Figure 5.16 shows the leakage resistance, $\mathrm{R}_{\mathrm{LEAK}}$, for the two $500 \mu \mathrm{m}$ devices.

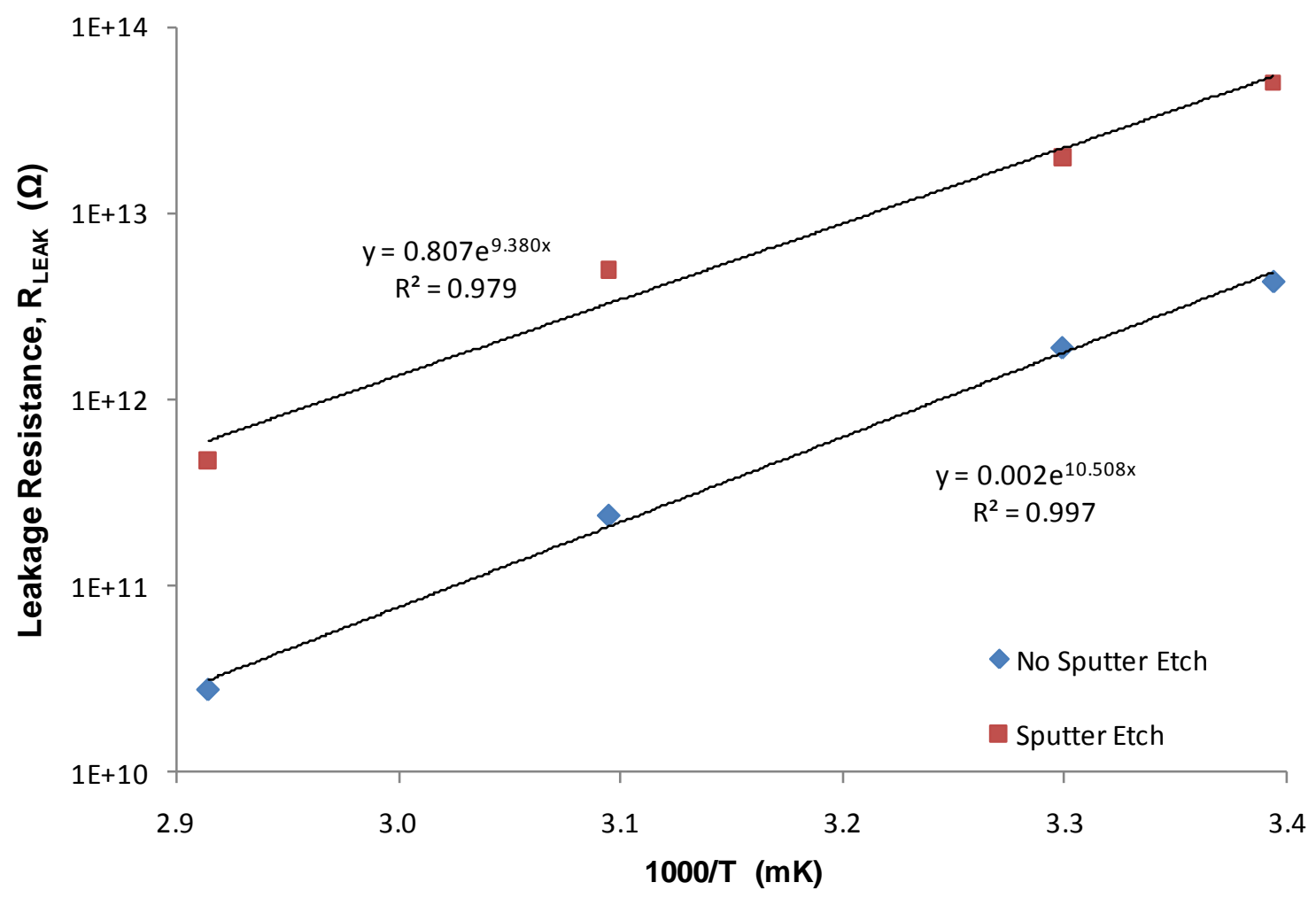

Figure 5.16 Leaky Schottky Barrier Resistance for $500 \mu \mathrm{m}$ Devices

The leakage resistance for both devices fits an exponential dependence, and should be inversely proportional to defect ionization (carrier concentration at the contact):

$$
R_{L E A K} \propto \frac{1}{N_{t}^{-}} \approx \exp \left(\frac{E_{t}}{k T}\right)
$$

where $N_{t}^{-}$is the ionized defect density and $\mathrm{E}_{\mathrm{t}}$ is the defect energy level referenced to the valence band. Table 5.5 gives the extracted defect energy levels for each device and offers possible explanations for the defect levels. 
Table 5.5 Defect Levels Accounting for Barrier Leakage Resistance

\begin{tabular}{|c|c|c|}
\hline & Defect Energy Level & Possible Defect Cause \\
\hline No Sputter Etch & $0.91 \mathrm{eV}$ & $\begin{array}{c}\text { nickel or vanadium impurity [41] } \\
\text { Cd vacancy [42] }\end{array}$ \\
\hline Sputter Etch & $0.81 \mathrm{eV}$ & $\begin{array}{c}\text { tin impurity [41] } \\
\text { Cd vacancy, Te anti-site [43] }\end{array}$ \\
\hline
\end{tabular}

The lower leakage resistance in the no sputter etch device supports the leaky barrier hypothesis; the higher leakage resistance in the sputter etch device suggests a more homogeneous barrier. From this model, we can speculate that the sputter etch decreased surface defect density, thus improving Schottky contact formation. 


\section{NUMERICAL MODELING}

The resistivity of $\mathrm{Cl}$-compensated $\mathrm{CdTe}$ is controlled by trap states near midgap [15]. Previous studies report trap densities from $10^{12}$ to $10^{16} \mathrm{~cm}^{-3}$ [41], [44] and carrier lifetimes from $5 \mu \mathrm{s}$ to $0.01 \mu \mathrm{s}$ [9], [18], [45]. Numerical models are used to investigate effects of the anode and cathode work functions on the current-voltage characteristic over a range of trap configurations (type, density, and energy level) and carrier lifetimes. The goal of this chapter is provide insight into how the contacts affect device performance, in particular the reverse leakage current. By comparing the simulations to measured data, a better understanding of the device physics can be achieved.

\subsection{Simulation Experiments}

Chapter 3 shows the effects of varying anode and cathode barriers at low voltage without traps; at low voltages the I-V curves are less affected by series resistance, making this voltage range important for determining the dominant conduction mechanisms. Forward bias current increases as the anode hole barrier the cathode electron barrier increase. The higher barriers create a large electron accumulation at the anode and a large hole accumulation at the cathode, increasing carrier injection. Reverse bias current decreases as both barriers increase, since large barriers block electron and hole currents 
from the metal to semiconductor. However, these simple relations do not hold when traps are added to the low voltage model.

Traps are defined by their energy level with respect to the conduction and valence bands: donors are referenced to the valence band and acceptors to the conduction band, as shown in figure 6.1.

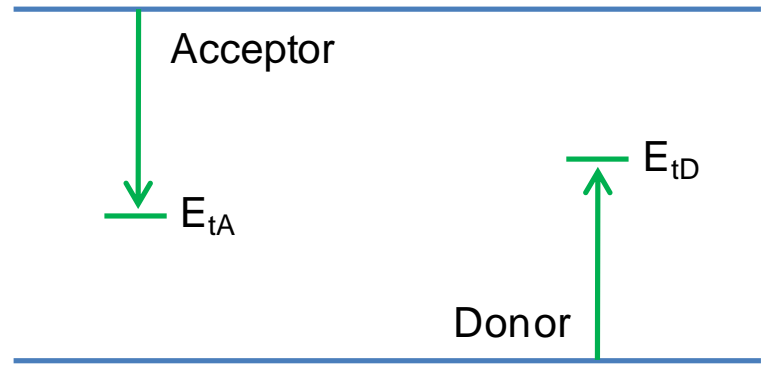

Figure 6.1 Trap Energy Level Definitions

Like dopants, ionized donor traps add free electrons to the conduction band, and ionized acceptor traps add holes to the valence band. It is necessary to continually verify the bulk carrier concentrations when changing trap properties, since room temperature resistivity must remain $\rho>10^{9} \Omega \cdot \mathrm{cm}$, as specified by the CdTe supplier. In a system with only one trap type, the resistivity guideline significantly limits the maximum trap density. In a device with both donor and acceptor traps, the traps compensate each other allowing for high trap densities yet low carrier concentrations, consistent with the resistivity requirement; this is also true for traps compensated by dopants. Ionized traps add fixed charge to the device changing the width of the depletion region at the Schottky contact; figure 6.2 shows the simulated device structure, and figure 6.3 shows the energy band diagram in a two-dimensional cut. 


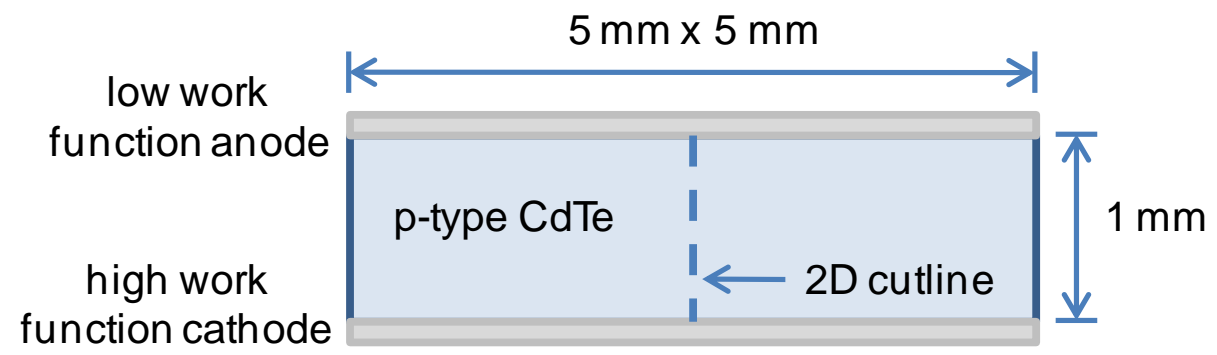

Figure 6.2 Simulated Device Structure

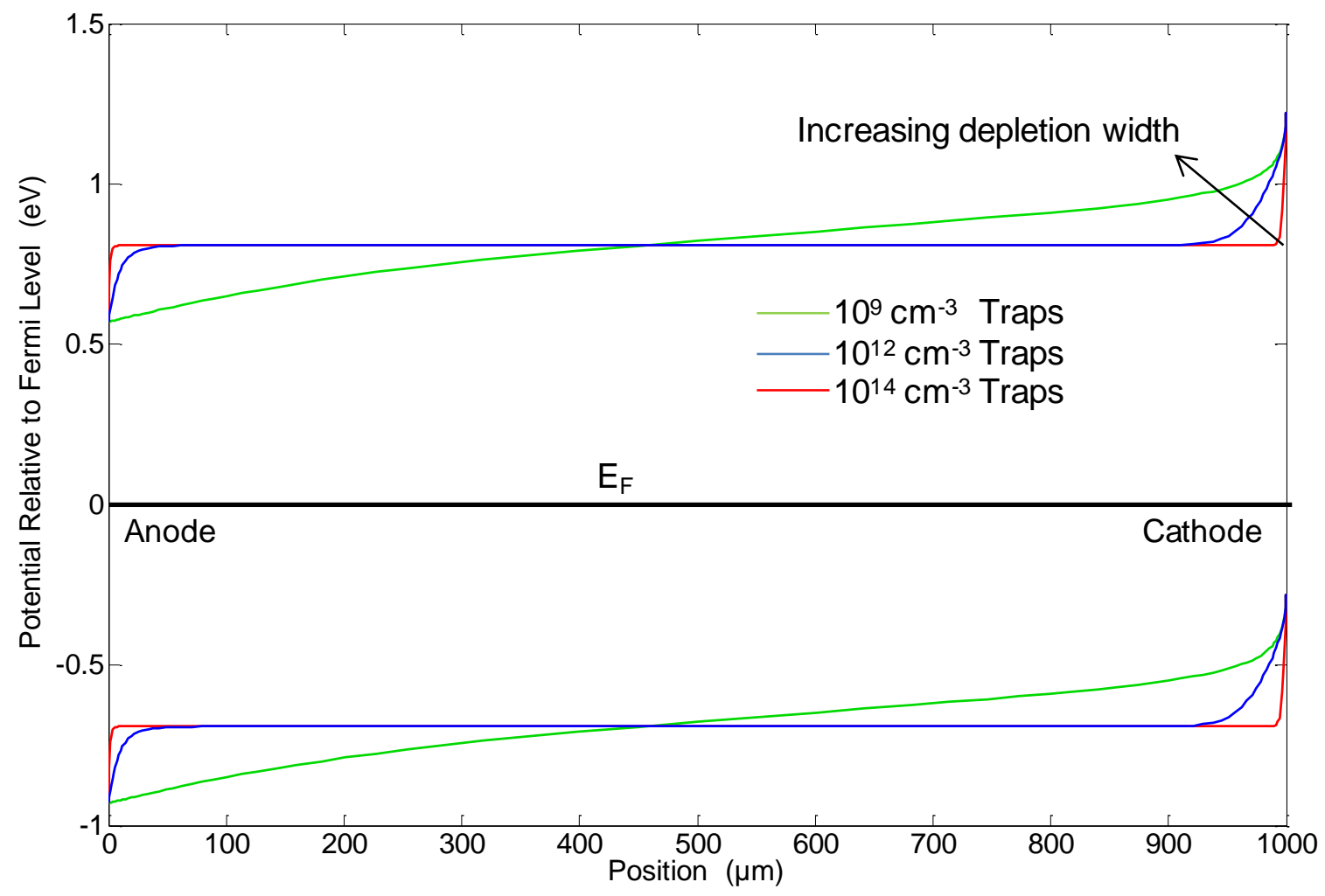

Figure 6.3 Depletion Region Widths at Zero Bias

Due to the varying depletion region width, the effects of anode and cathode work functions change with trap density.

To further investigate the effects of carrier lifetime, trap energy, and trap density, the matrix of simulations in table 6.1 was performed for both donor and acceptor traps. 
Table 6.1 Simulation Matrix

\begin{tabular}{|c|c|c|c|}
\hline Parameter & Min & Max & Step \\
\hline Anode work function & $4.65 \mathrm{eV}$ & $5.05 \mathrm{eV}$ & $0.2 \mathrm{eV}$ \\
\hline Trap energy & $0.65 \mathrm{eV}$ & $0.85 \mathrm{eV}$ & $0.1 \mathrm{eV}$ \\
\hline Trap density & $10^{9} \mathrm{~cm}^{-3}$ & $10^{14} \mathrm{~cm}^{-3}$ & factor of 10 \\
\hline Carrier lifetime & $10^{-7} \mathrm{~s}$ & $10^{-6} \mathrm{~s}$ & $5 \times 10^{-7} \mathrm{~s}$ \\
\hline Doping concentration & $7 \times 10^{7} \mathrm{~cm}^{-3}$ & $10^{13} \mathrm{~cm}^{-3}$ & - \\
\hline
\end{tabular}

Carrier lifetime and capture cross section are inversely related, and only one needs to be specified in a trap definition; carrier lifetime is specified since it is more prevalent in the literature. The step for varying doping concentration is not indicated, since it changes with trap configuration in order to maintain acceptable bulk resistivity. The cathode work function was fixed at $\phi_{\mathrm{m}}=5.50 \mathrm{eV}$, corresponding to an electron barrier $\phi_{\mathrm{Bn}}=1.22 \mathrm{eV}$ or hole barrier $\phi_{\mathrm{Bp}}=0.28 \mathrm{eV}$; with traps present, the cathode has no effect as long as it is a low barrier to holes. The test matrix results are described qualitatively, since resistivity changes with trap configuration; any quantitative comparisons would show the effects of changing carrier concentrations rather than device configurations.

\subsubsection{Trap Density}

The ionized trap density is affected by carrier lifetime, trap energy level, and trap density. Acceptor traps closer to the valence band and donor traps closer to the conduction band have a higher ionization probability. The effect of trap level is difficult to separate from resistivity, since changes in trap level can significantly affect carrier concentrations. However, trap level affects device performance similarly to trap density, so traps will be defined at midgap, $0.75 \mathrm{eV}$, for the following simulations. 
Systems with two traps or one trap compensated by doping perform very similarly; two trap systems have a slightly higher recombination rate, resulting in more generation in reverse bias and more recombination in forward bias. The difference in

device performance is small, since the trap density is nearly the same; for the following qualitative discussion, the exact trap configuration is not significant.

\subsubsection{Low Trap Density}

Without traps the anode hole barrier controls electron currents in forward bias and hole currents in reverse bias; for the anode barrier to control reverse bias current, the cathode must have a high electron barrier to block metal-semiconductor electron current at the cathode. Figure 6.4 shows the simulated I-V characteristic for a device with no traps and cathode work function $\phi_{\mathrm{m}}=5.50 \mathrm{eV}$, corresponding to an electron barrier, $\phi_{\mathrm{Bn}}=1.22 \mathrm{eV}$. This same cathode work function is used for all figures in this section. 


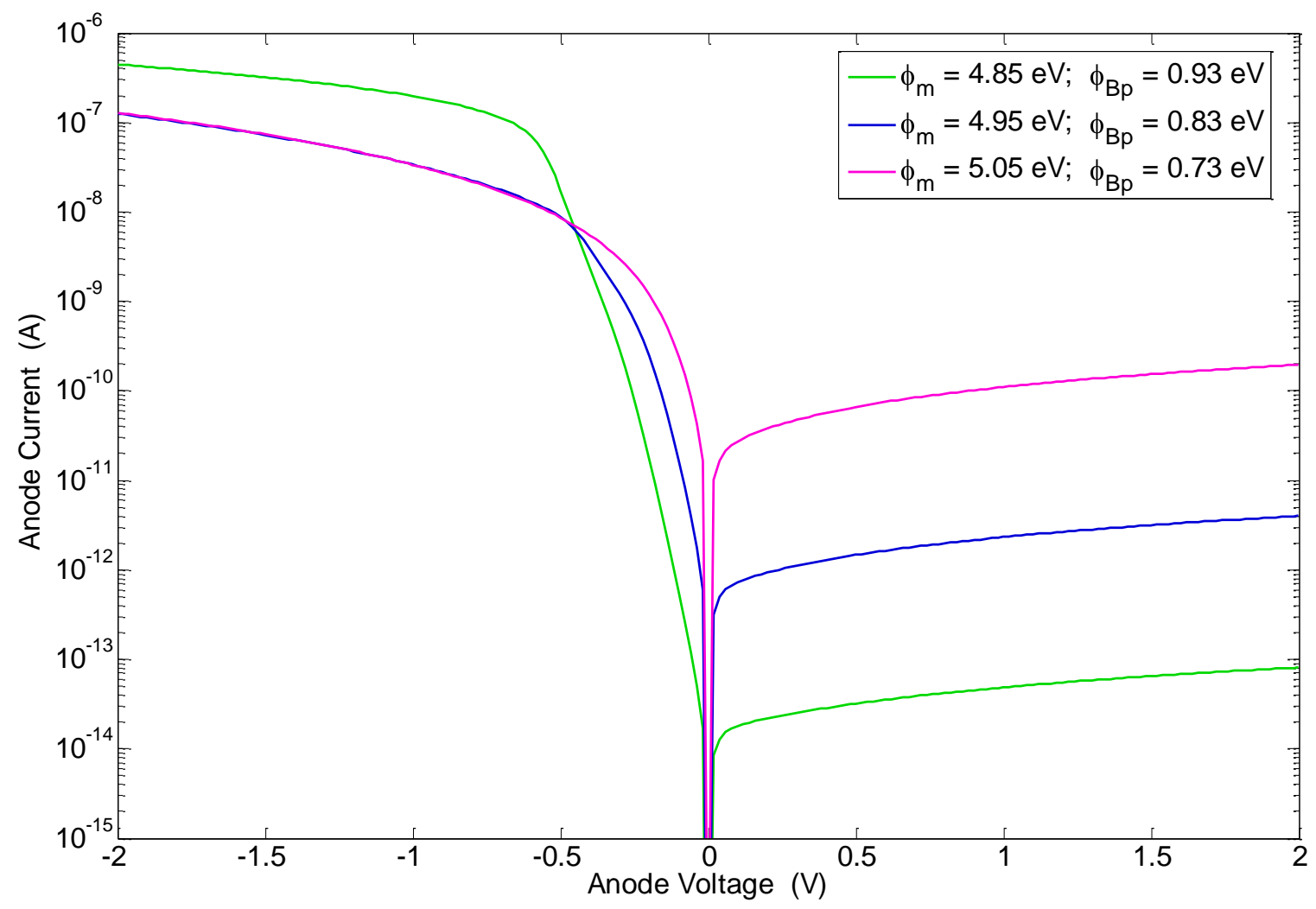

Figure 6.4 I-V Characteristics for Device without Traps with Varying Anode Work Function

Adding traps significantly changes the effect of the anode work function; figure 6.5 shows the I-V characteristics for devices with $10^{9} \mathrm{~cm}^{-3}$ acceptor traps and $7 \times 10^{7} \mathrm{~cm}^{-3}$ acceptor dopants. In forward bias, electron current dominates. Increasing the anode hole barrier beyond $0.83 \mathrm{eV}$ does not decrease the reverse bias current, since electron current dominates at this point. 


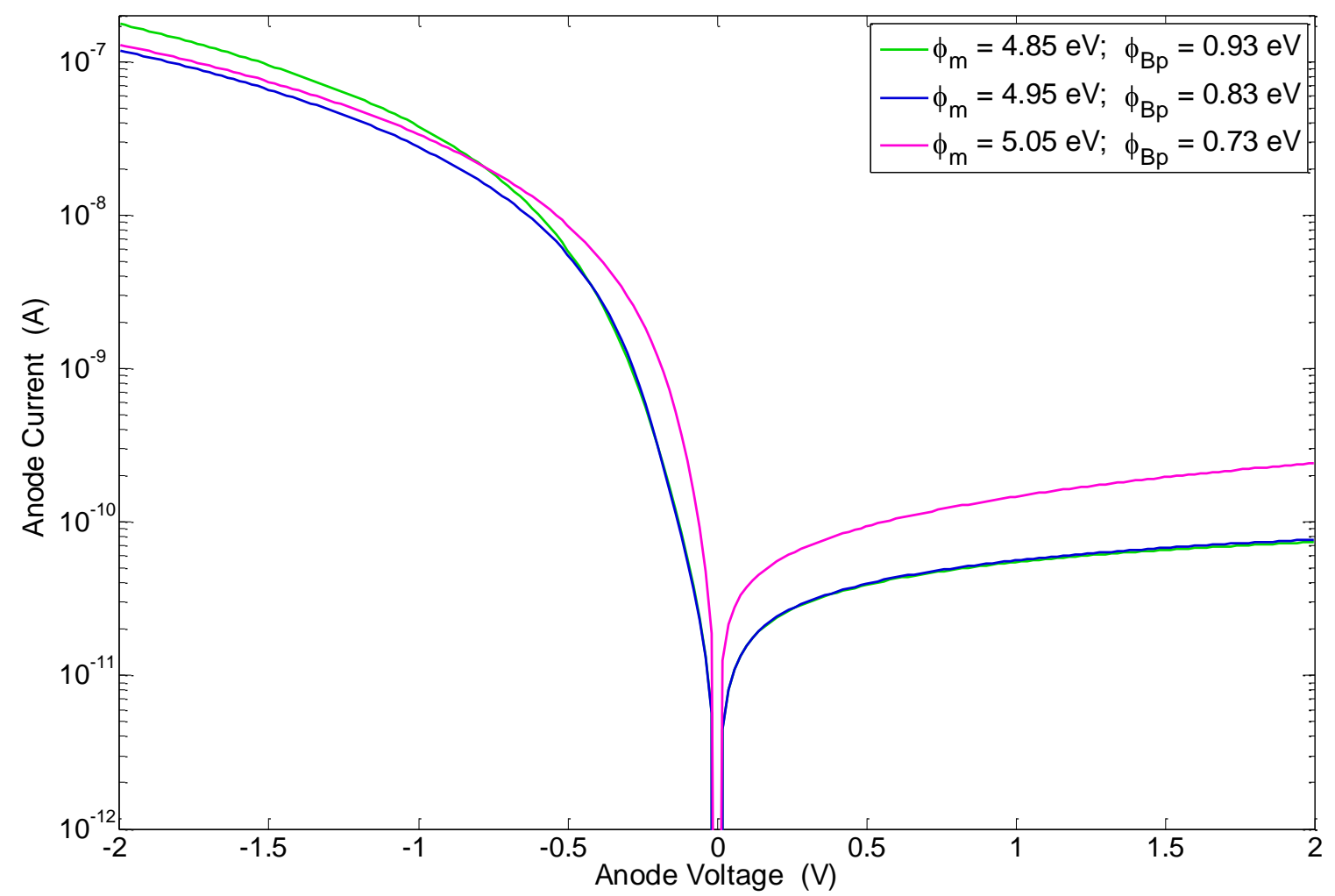

Figure 6.5 I-V Characteristics for Low Trap Density Device with Varying Anode Work Function

The energy band diagram in figure 6.6 shows the reverse bias currents for the device in figure 6.5, with $10^{9} \mathrm{~cm}^{-3}$ acceptor traps and $7 \times 10^{7} \mathrm{~cm}^{-3}$ acceptor dopants. 


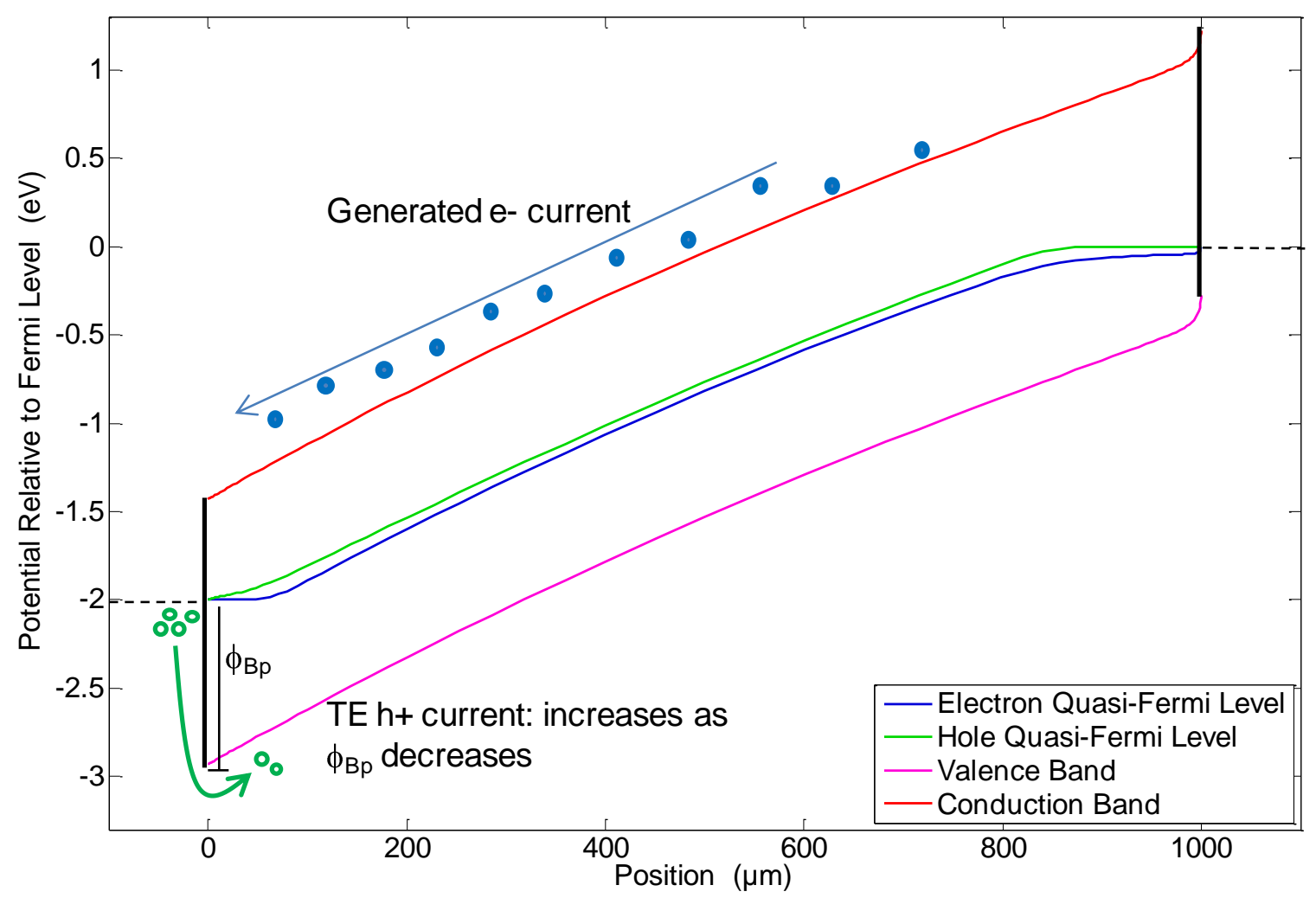

Figure 6.6 Low Trap Density Energy Band Diagram at Reverse Bias $(+2 V)$

The depletion region is large at low trap density, so there is a high generated electron current in reverse bias; the anode has no effect on the generated electron current, and since it is generated in the semiconductor and swept to the anode, the cathode work function does not affect this current. However, when the anode hole barrier becomes small enough, hole current over the metal to semiconductor barrier begins to dominate; the cathode does not affect this current until the cathode hole barrier increases enough to reverse device polarity. The energy band diagram in figure 6.7 shows the forward bias currents for the same device with $10^{9} \mathrm{~cm}^{-3}$ acceptor traps and $7 \times 10^{7} \mathrm{~cm}^{-3}$ acceptor dopants. 


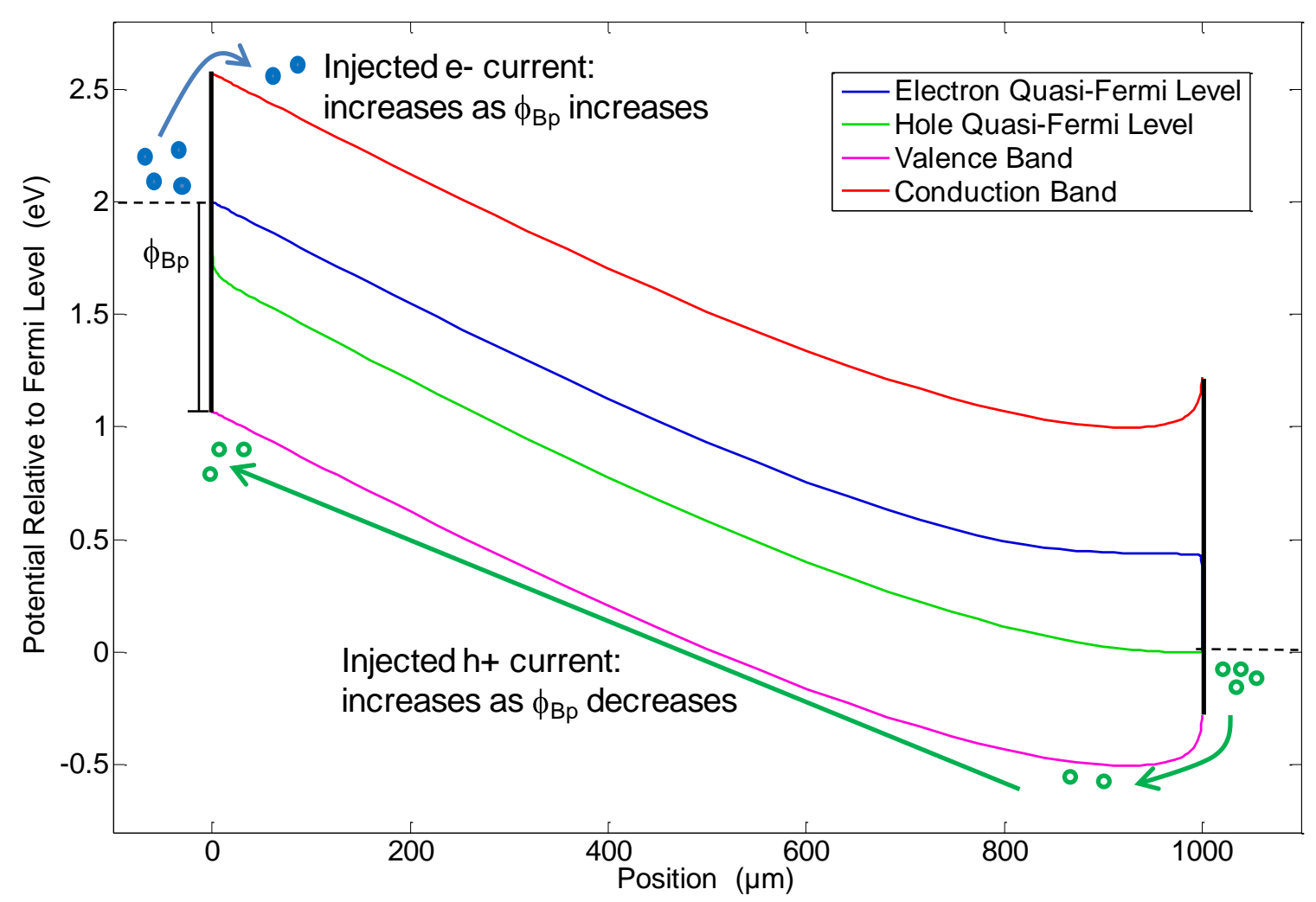

Figure 6.7 Low Trap Density Energy Band Diagram at Forward Bias (-2V)

In forward bias, a higher anode hole barrier (lower electron barrier) allows more electrons to be injected across the depletion region; the semiconductor-metal electron barrier at the cathode has no effect since the electrons injected from the anode recombine before reaching the cathode. Decreasing the anode hole barrier increases anode hole current from semiconductor to metal until it dominates over the injected electron current; the cathode hole barrier does not affect this current until it becomes large enough to reverse device polarity. 


\subsubsection{High Trap Density}

Figure 6.8 shows typical I-V characteristics for a device with $10^{14} \mathrm{~cm}^{-3}$ acceptor traps and $10^{13} \mathrm{~cm}^{-3}$ donor dopants; figures 6.9 and 6.10 show the energy band diagram for this device in reverse and forward bias with currents indicated.

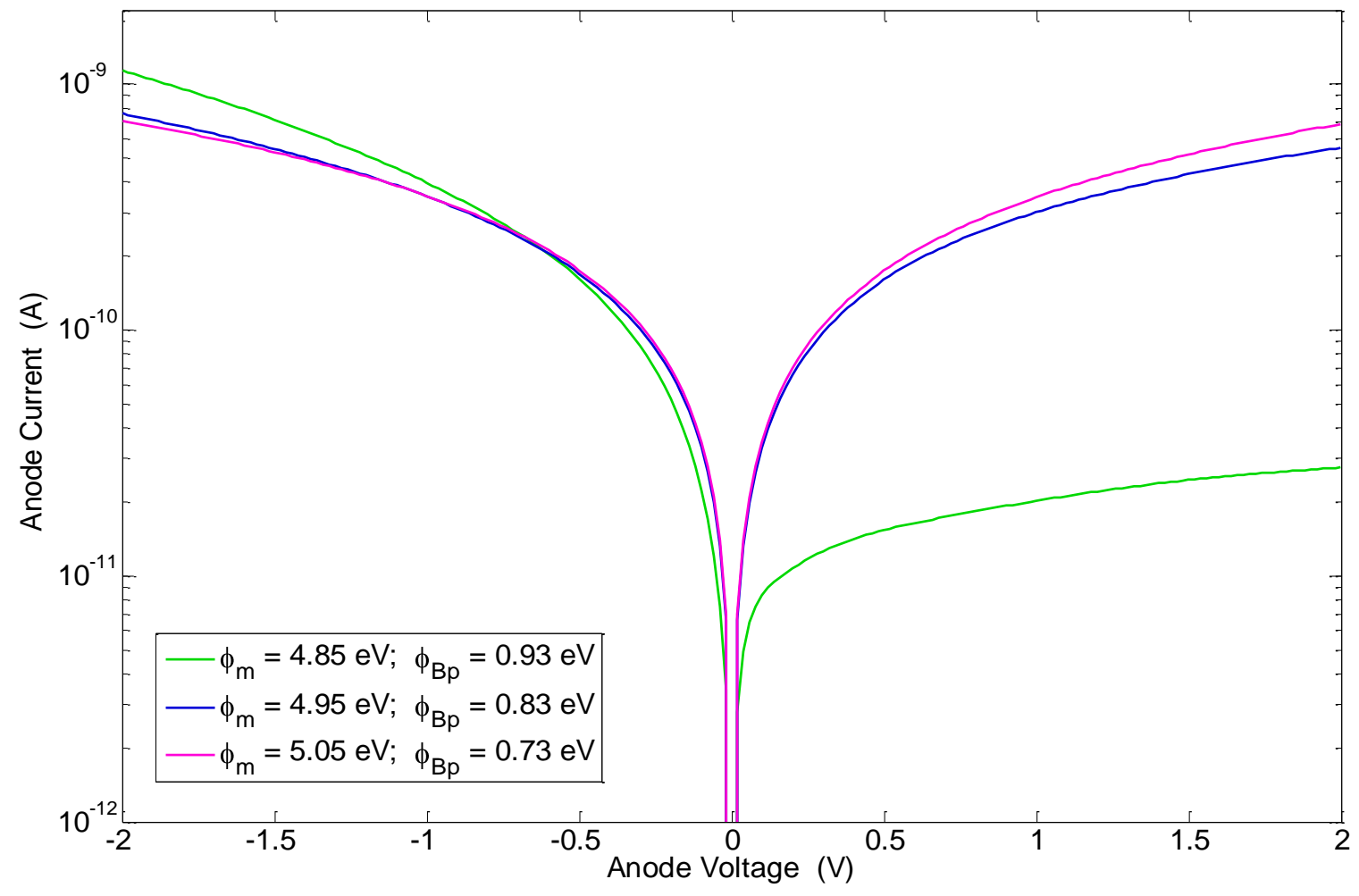

Figure 6.8 I-V Characteristics at High Trap Density with Varying Anode Work Function 


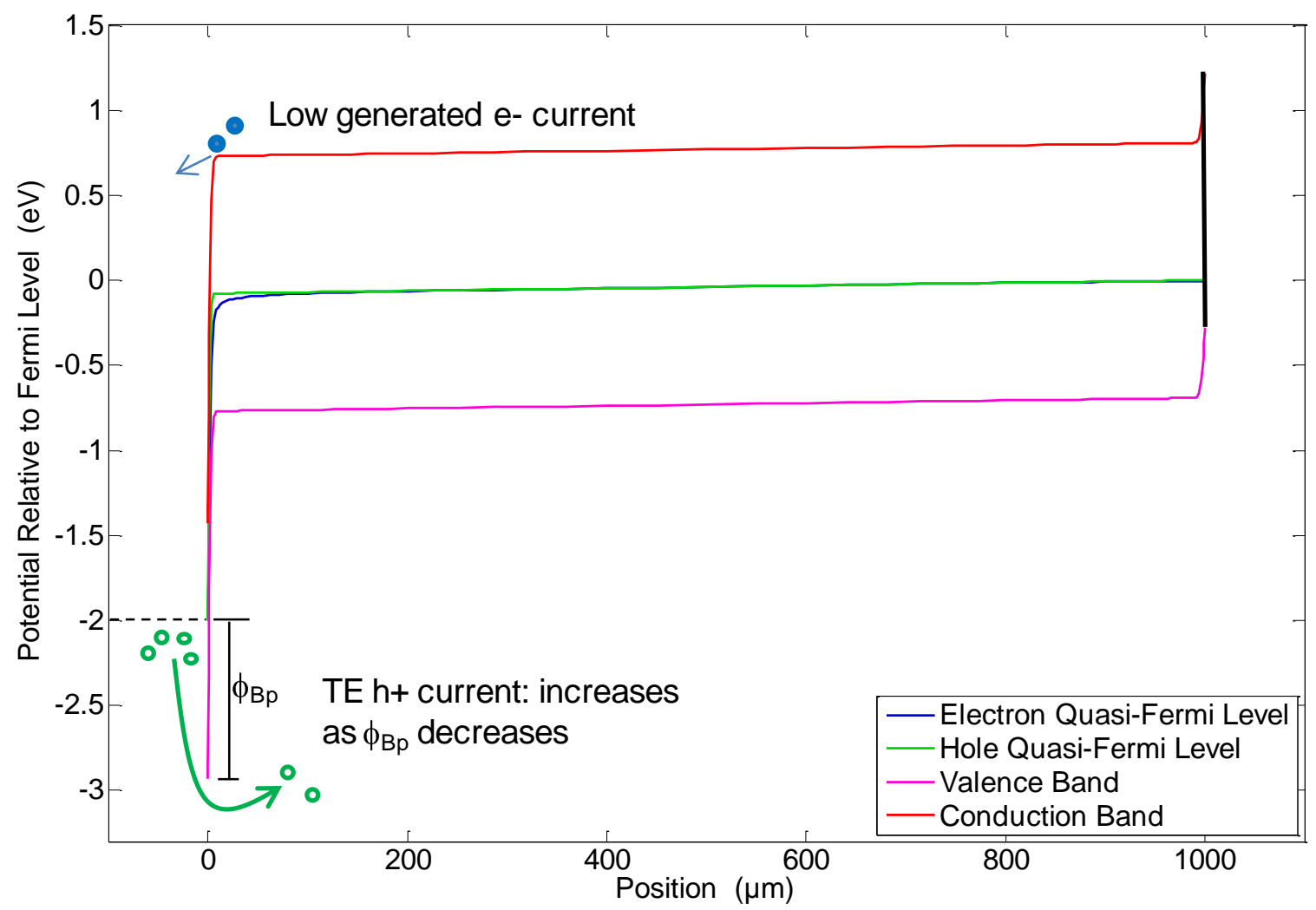

Figure 6.9 High Trap Density Energy Band Diagram in Reverse Bias (+2V)

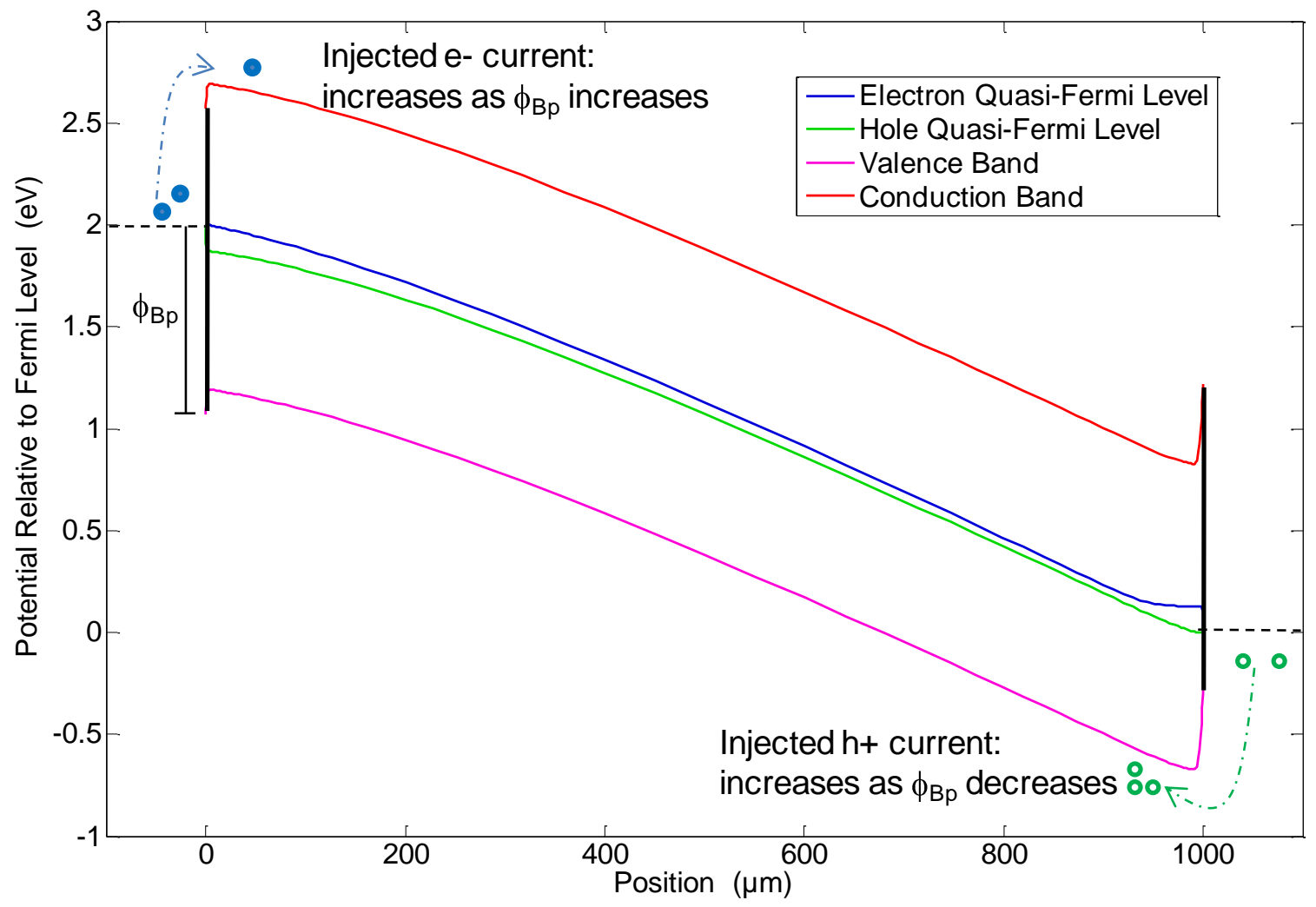

Figure 6.10 High Trap Density Energy Band Diagram in Forward Bias (-2V) 
High trap densities decrease the depletion region width; this yields a lower generated electron current in reverse bias [17]:

$$
I_{G E N}=\frac{q n_{i} W}{\left(\tau_{n} \tau_{p}\right)^{1 / 2}}
$$

where $\mathrm{W}$ is the depletion width, $\mathrm{n}_{\mathrm{i}}$ is the intrinsic carrier concentration, and $\tau_{\mathrm{n}}$ and $\tau_{\mathrm{p}}$ are the carrier lifetimes. The lower generation current makes the anode metal-semiconductor hole current dominant, so the anode hole barrier strongly controls the reverse bias current, until hole current decreases below the level of the generated electron current; the cathode hole barrier does not affect the anode hole current until it becomes comparable to the anode hole barrier. In forward bias, the anode hole barrier minimally affects the forward current: electron current decreases as hole current increases, yielding only a slight change in I-V shape; as usual, the cathode has no effect until it becomes a large enough hole barrier to block the metal-semiconductor hole current at the cathode.

\subsubsection{Carrier Lifetime}

Figure 6.11 shows the effect on the I-V characteristic of varying carrier lifetime, $\tau$; the simulated device contains $10^{12} \mathrm{~cm}^{-3}$ acceptor traps and $10^{11} \mathrm{~cm}^{-3}$ donor dopants; the anode work function is $\phi_{\mathrm{m}}=4.85 \mathrm{eV}\left(\phi_{\mathrm{Bp}}=0.93 \mathrm{eV}\right)$, and the cathode work function is $\phi_{\mathrm{m}}=5.35 \mathrm{eV}\left(\phi_{\mathrm{Bp}}=1.07 \mathrm{eV}\right)$. In this figure, electron current dominates in forward and reverse bias. 


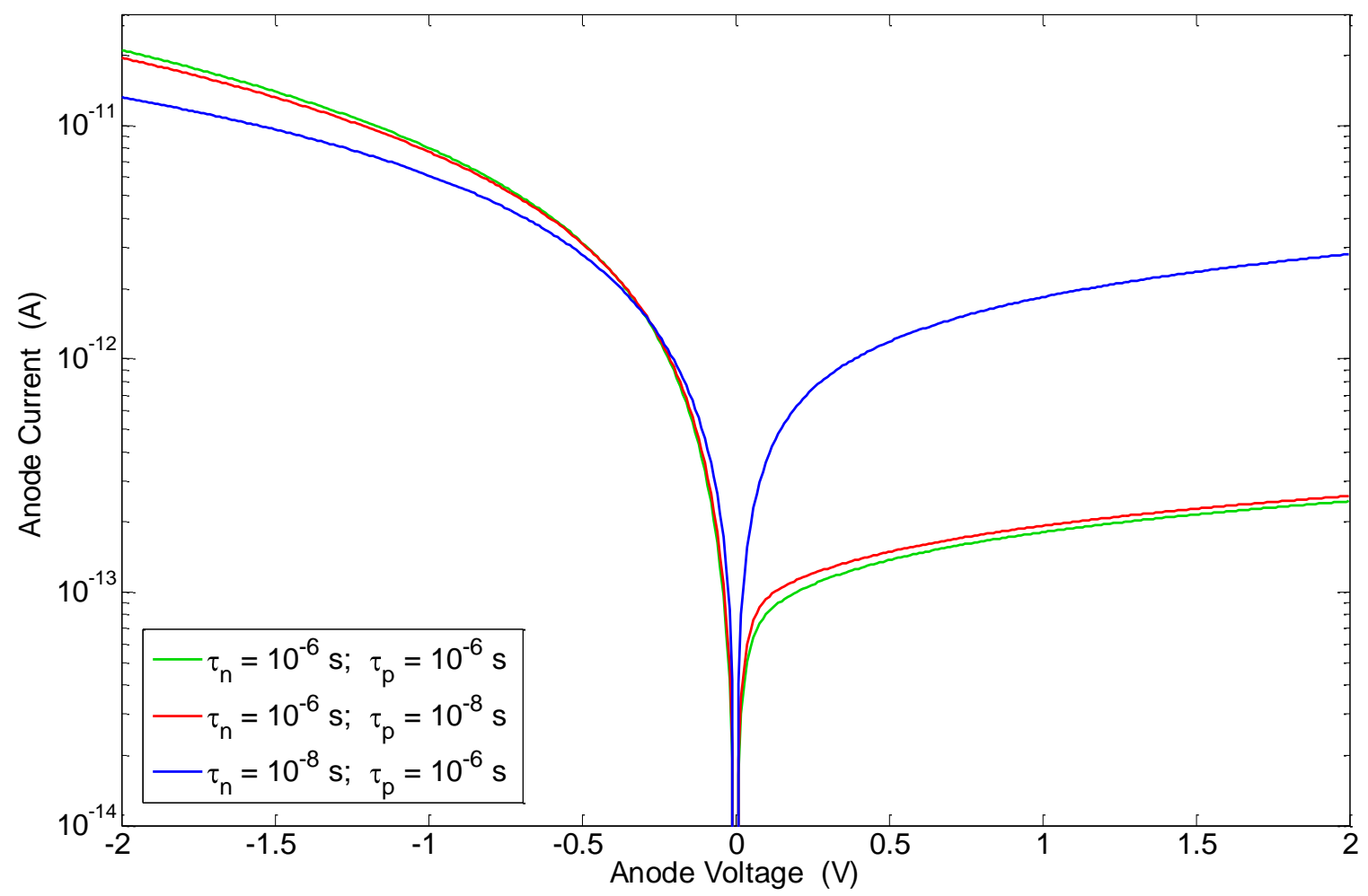

Figure 6.11 I-V Characteristics with Varying Carrier Lifetime

Carrier lifetime is inversely related to the generation-recombination rate. Decreasing the electron lifetime increases the electron capture and generation rates; this causes a large increase in reverse generation current (electron dominated). In forward bias, the electron current decreases, but the reason is less apparent. Acceptor traps ionize by capturing electrons or releasing holes. With a shorter electron lifetime, more electrons are captured, and the higher ionized trap density changes the band diagram; this change actually results in a lower injected electron current, as seen in Figure 6.11. Changing hole lifetime has the same effect on hole currents; since electron current dominates, this effect is not noticeable. The change in ionized trap due density to the shorter hole lifetime slightly affects the generation and recombination rates for electrons, and this is reflected in figure 6.11 . 


\subsubsection{Contact Area}

Determining the effect of decreased anode area is the last task before modeling the measured devices. Until now the contacts have been symmetric and have covered the full area of the device (blanket contacts), but the measured devices have a small anode contact and a blanket cathode. Since current is proportional to effective device area, decreasing the size of a contact decreases the current entering the device through it; hence asymmetrical contacts create an inherent rectification. With the metal work function at each contact set equal to the bulk Fermi level, figure 6.12 shows the contact asymmetry on the I-V curve; this rectification becomes much more significant with blocking contacts.

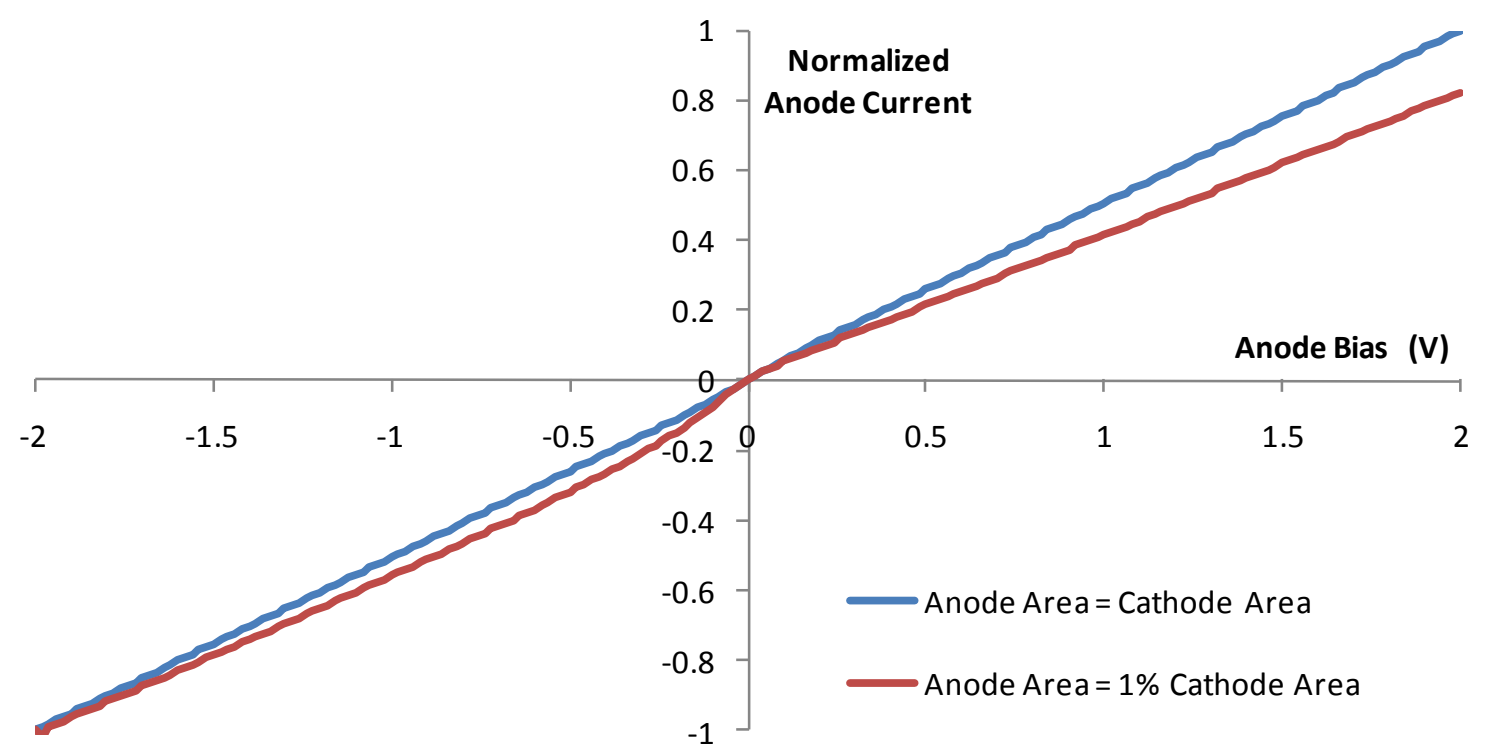

Figure 6.12 I-V Characteristics with Varying Anode Size 


\subsubsection{Simulation Experiment Summary}

Table 6.2 summarizes the effects changing various device parameters.

Table 6.2 Summary of Device Parameter Effects

\begin{tabular}{|c|c|c|}
\hline Parameter & Forward Bias & Reverse Bias \\
\hline Trap energy level & \multicolumn{2}{|c|}{ - Deeper traps have higher ionization probability } \\
\hline Low trap density & $\begin{array}{l}\text { - Injected e- current dominates with } \\
\text { high anode hole barrier } \\
\text { - Injected h+ current dominates } \\
\text { with low anode hole barrier }\end{array}$ & $\begin{array}{l}\text { - Generated e- current dominates } \\
\text { with high anode hole barrier } \\
\text { - Metal-semiconductor TE h+ } \\
\text { current dominates with low anode } \\
\text { hole barrier }\end{array}$ \\
\hline High trap density & $\begin{array}{l}\text { - Injected e- current dominates with } \\
\text { high anode hole barrier } \\
\text { - Injected h+ current dominates } \\
\text { with low anode hole barrier }\end{array}$ & $\begin{array}{l}\text { - Decreased depletion region width } \\
\text { yields lower generated e- current } \\
\text { - Metal-semiconductor TE h+ } \\
\text { current increases with low anode } \\
\text { hole barrier and dominates over } \\
\text { large range of barrier heights }\end{array}$ \\
\hline Cathode work function & \multicolumn{2}{|c|}{ - No effect until hole barrier large enough to reverse device polarity } \\
\hline Carrier lifetime & \multicolumn{2}{|c|}{$\begin{array}{l}\text { - Inversely proportional to recombination rate } \\
\text { - Decreasing lifetime increases reverse bias generation } \\
\text { - Changing carrier lifetime changes the ionized trap density, thus changing } \\
\text { the shape of the energy bands }\end{array}$} \\
\hline Contact area & \multicolumn{2}{|c|}{$\begin{array}{l}\text { - Current is proportional to contact area } \\
\text { - Asymmetric contacts create inherent rectification }\end{array}$} \\
\hline
\end{tabular}

\subsection{Comparison with Device Measurements}

Initially a low voltage model was determined for the no sputter etch device without traps. This model dominated by thermionic emission did not match measured data at low voltages, as expected from the high ideality factor extracted from the measurements. Adding traps to the model produces a good fit to measured data at room 
temperature, but this model does not have sufficient current growth with increasing temperature. Implementing a temperature dependent bandgap improves agreement between the numerical model and measurements [46]:

$$
E_{G}=E_{G 0}-\frac{\alpha T^{2}}{\beta+T}
$$

Gilliland et al and Yamanaka et al have investigated the bandgap of CdTe over large temperature ranges [47], [48]; whereas Kosyachenko et al model the bandgap in order to accurately describe their results [22]. Figure 6.13 shows three models for bandgap temperature dependence in the region of interest; also shown is the model used in this thesis, which was chosen to best fit the measured data for the no sputter etch device.

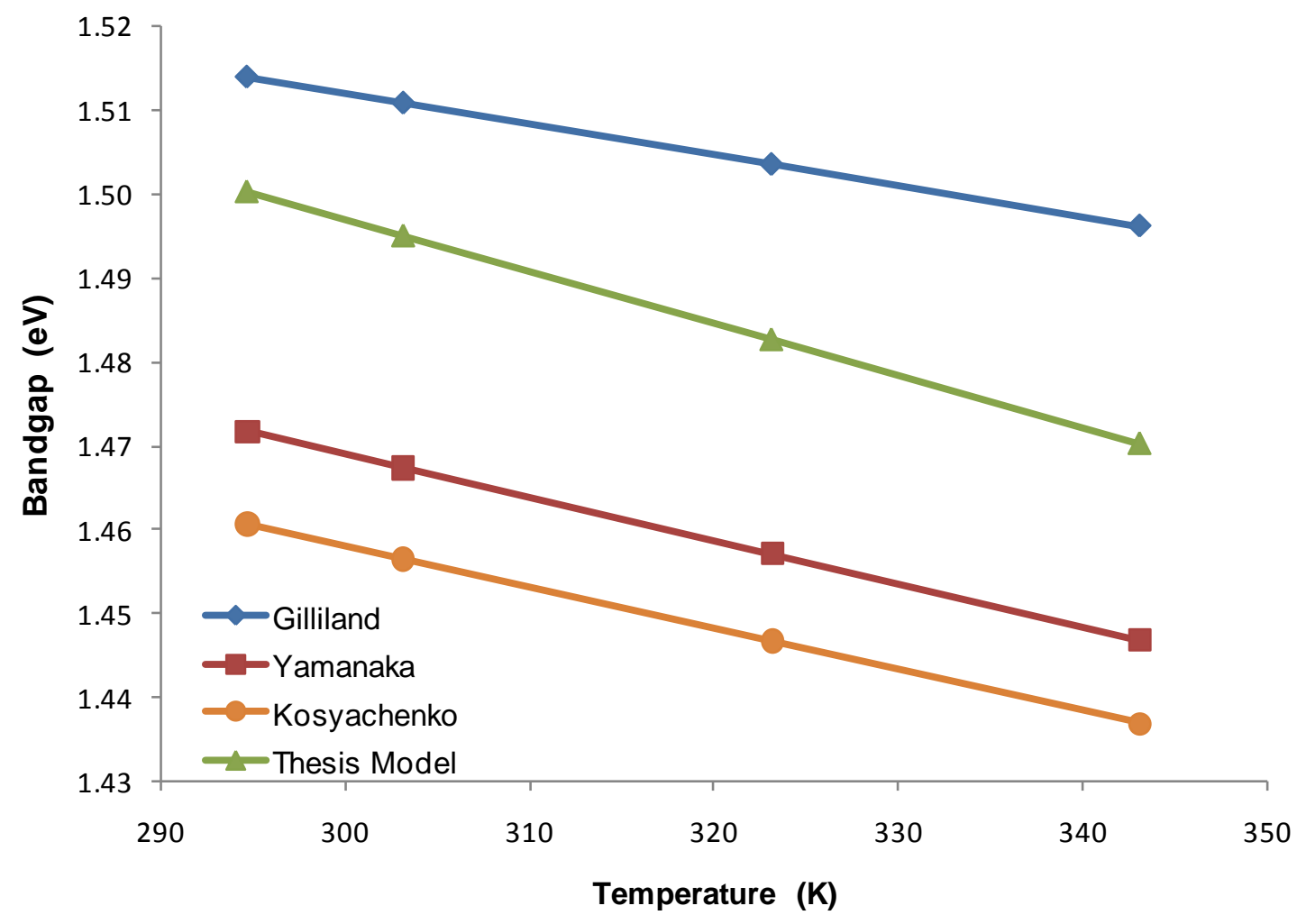

Figure 6.13 Bandgap Temperature Dependence 
High electric fields can cause drift velocity saturation, where drift current is independent of applied bias. The literature shows no drift velocity saturation up to $2 \mathrm{kV} / \mathrm{cm}$ for Acrorad Cl-compensated CdTe [21], but other authors indicate a slight decrease in electron mobility above $12 \mathrm{kV} / \mathrm{cm}$ for high resistivity CdTe [49]. Due to low hole mobility, information on hole drift velocity is limited; these numerical models assumes hole velocity saturation at the same applied field as electron velocity saturation, similar to GaAs, Ge, and Si [17], [50], [51]. Incorporating field dependent mobility into the present model decreases current in the numerical model at high reverse bias, since carrier velocity begins to saturate near the anode contact edges.

Figure 6.14 shows the high voltage simulated and measured results for the $500 \mu \mathrm{m}$ no sputter etch device, and table 6.3 gives the model parameters. This model uses the material parameters in tables 3.1 and 3.2 (pages 15 and 16); appendix A contains the simulation code.

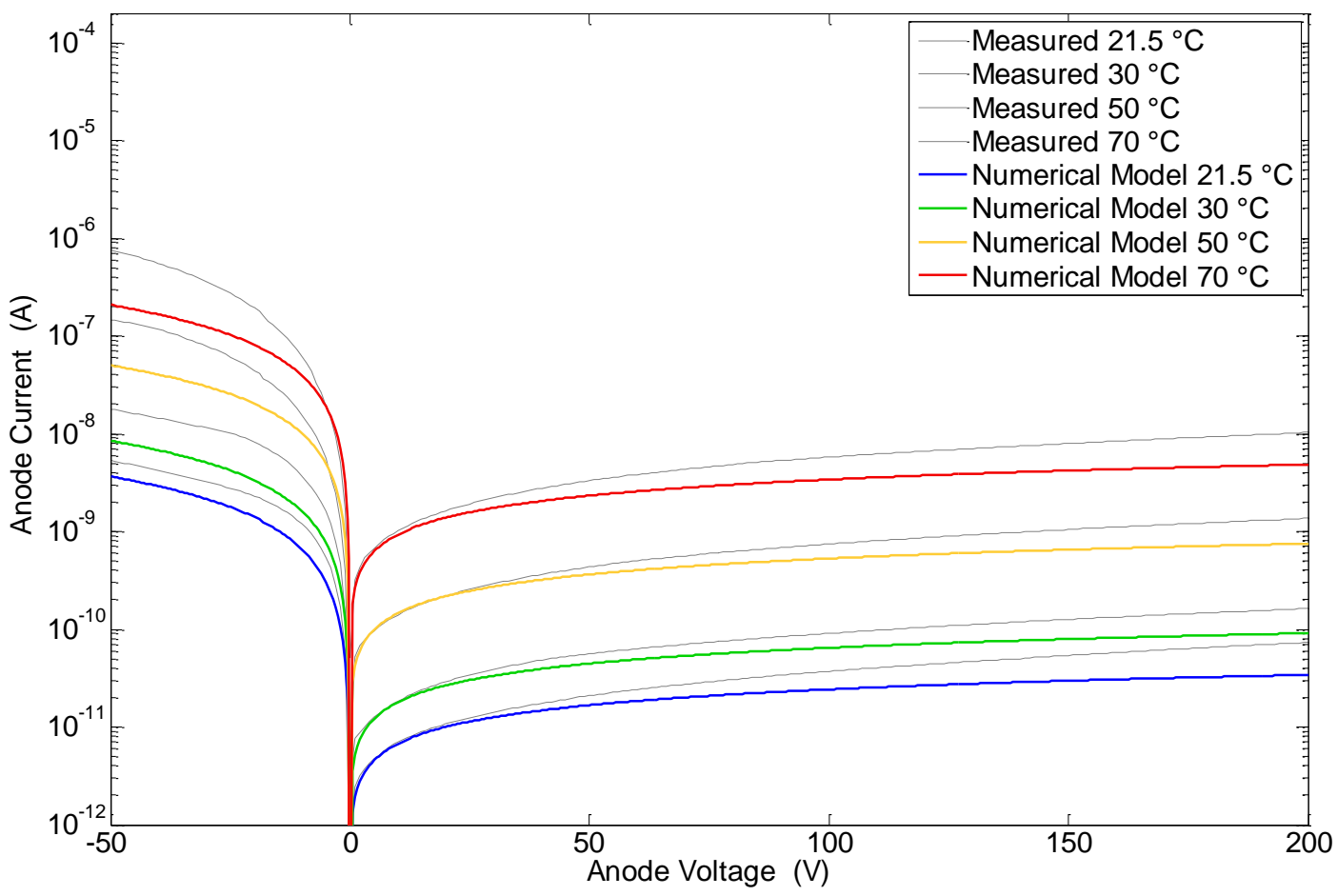

Figure 6.14 High Voltage I-V Characteristic for No Sputter Etch Device 
Table 6.3 Numerical Model Parameters for $500 \mu \mathrm{m}$ No Sputter Etch Device

\begin{tabular}{|l|l|}
\hline Anode work function & $4.996 \mathrm{eV}\left(\phi_{\mathrm{Bp}}=0.781 \mathrm{eV} @ 300 \mathrm{~K}\right)$ \\
\hline Cathode work function & $5.15 \mathrm{eV}$ \\
\hline Bandgap at $300 \mathrm{~K}$ & $1.497 \mathrm{eV}$ \\
\hline Fermi level & $\mathrm{E}_{\mathrm{F}}-\mathrm{E}_{\mathrm{Fi}}=0.11 \mathrm{eV}$ \\
\hline Traps & acceptors, $3 \times 10^{11} \mathrm{~cm}^{-3}, \mathrm{E}_{\mathrm{C}}+0.75 \mathrm{eV}$ \\
\hline Compensation & donors, $4 \times 10^{9} \mathrm{~cm}^{-3}$ \\
\hline
\end{tabular}

Increasing the anode barrier height from $0.781 \mathrm{eV}$ to $0.81 \mathrm{eV}$ yields an approximate model for the sputter etch device. Figure 6.15 shows the high voltage simulated and measured results for the $500 \mu \mathrm{m}$ sputter etch device.

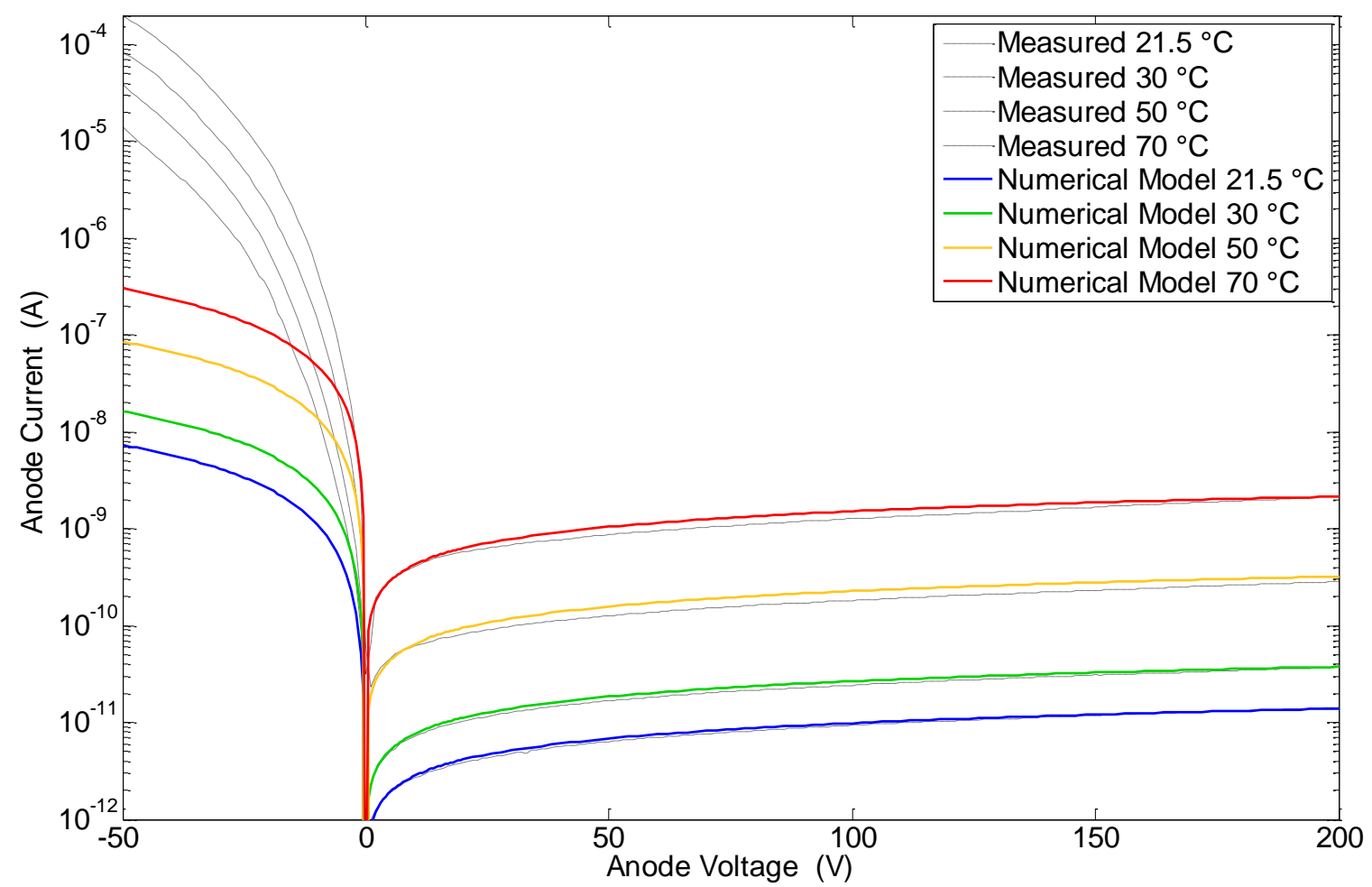

Figure 6.15 High Voltage I-V Characteristic for Sputter Etch Device 
While the numerical models shown in figures 6.14 and 6.15 show the correct current magnitude in reverse bias, they do not correctly describe forward bias. In both models, hole current injected from the cathode dominates forward bias current; hole current over the anode metal-semiconductor barrier dominates reverse bias current. Electron current does not contribute significantly in either case.

The forward bias current of no sputter etch device is nearly described by the numerical model in figure 6.14. By increasing anode work function and increasing carrier concentration slightly, the model would fit measured data at room temperature $\left(21.5^{\circ} \mathrm{C}\right)$ and $30{ }^{\circ} \mathrm{C}$; however, the slope of simulated data in forward bias does not match that of the measured data, at higher temperatures. This is likely due to insufficient electron current injected at the anode: electron mobility decreases less with temperature than hole mobility, so the electron current is more significant at high temperature. In reverse bias, this model only matches at low voltage, but it is clearly missing a linear component at higher voltages.

The numerical model in figure 6.5 accurately describes the sputter etch device in reverse bias, since the measured data does not show a large linear component, even at high bias. However, the sputter etch device measurements show three orders of magnitude higher forward bias current than the numerical model. Since the surface is not homogeneous, the effective cathode work functions could be different. Increasing cathode work function increases the forward bias current slope, since more holes are injected at the cathode; but this does not resolve the discrepancy between the model and measured data. Changes to trap or doping concentrations in the bulk are not justified, since surface treatments are the only fabrication difference. 
Analyzing the current versus voltage measurements in chapter 5 yielded significantly different differential resistance in the two devices, as shown in figure 5.11. This result suggested high injected electron currents, similar to the literature [39]. Changing the trap and compensation densities can increase electron concentration and modify recombination currents in forward bias; higher electron concentration would yield increased electron recombination and higher electron injection at the anode, while still

maintaining the required resistivity $\rho>10^{9} \mathrm{~cm}^{-3}$. Ongoing simulations indicate that this will yield a much more accurate model for both devices in forward bias; however, the numerical model accuracy will always be limited by its complexity. The model does not account for a native oxide, inhomogeneous barrier, interface states, or changes in surface stoichiometry. Since the sputter etch is believed to modify these properties [26], future numerical models must include these factors.

\subsection{Resolving Differences in Numerical and Analytical Models}

Taking a step back from the complex devices models and considering the voltage dependence of the two devices provides insight into why the present analytical and numerical models do not agree. Figure 6.16 shows the voltage dependence of the sputter etch and no sputter etch devices. 


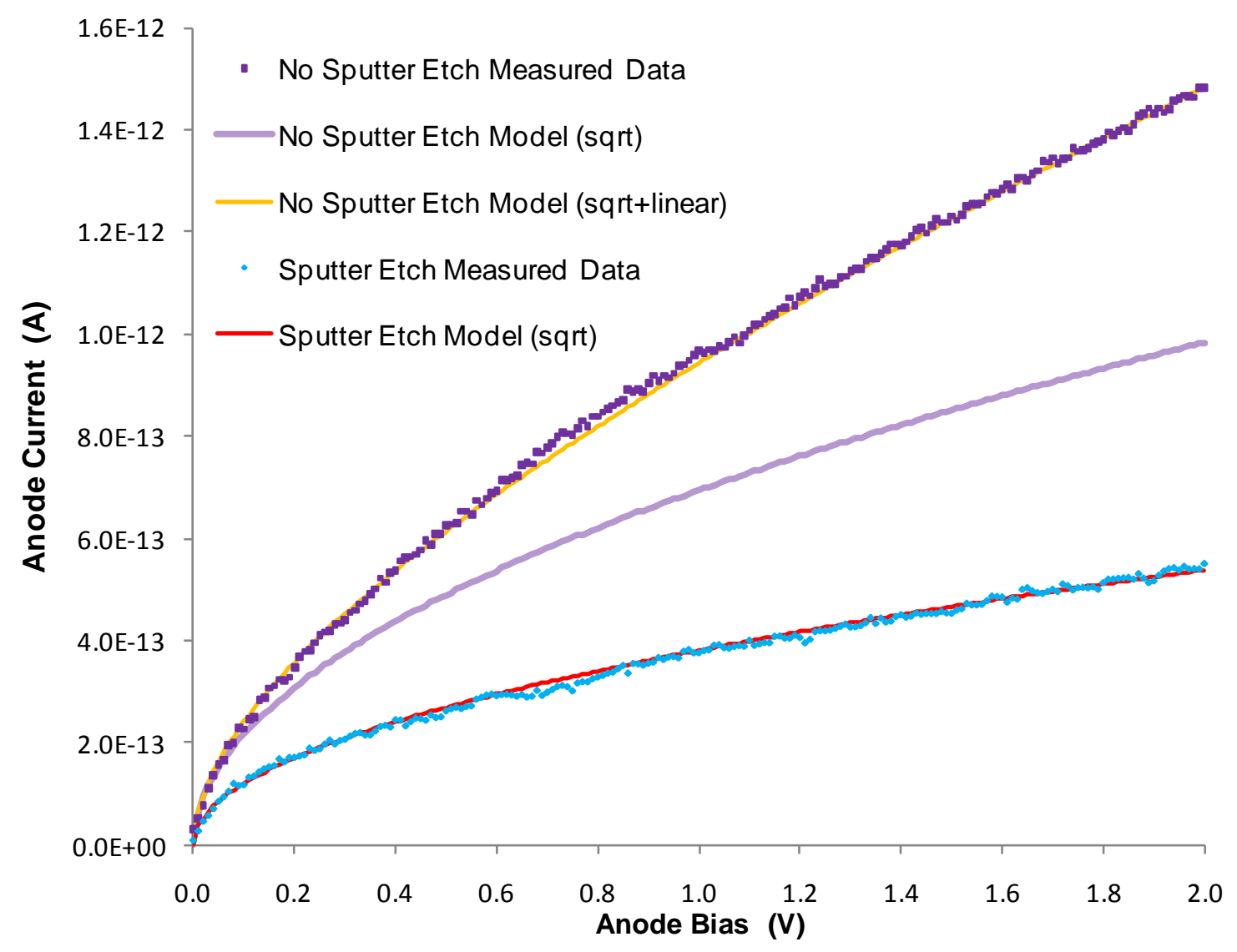

Figure 6.16 Voltage Dependence at Low Reverse Bias

The sputter etch device has $\sqrt{V}$ dependence, whereas the no sputter etch device has a similar $\sqrt{V}$ dependence plus a linear component. Table 6.4 lists the voltage and temperature dependence of possible current mechanisms.

Table 6.4 Voltage and Temperature Dependence of Possible Current Mechanisms

\begin{tabular}{|c|c|c|}
\hline Current mechanism & Voltage dependence & Temperature dependence \\
\hline Thermionic field emission & $\sqrt{V}$ & $T \exp (-1 / T)$ \\
\hline Diffusion & $\sqrt{V}$ & $T^{3 / 2} \exp (-1 / T)$ \\
\hline Generation & $\sqrt{V}$ & $\exp (-1 / T)$ \\
\hline Ohmic leakage & $V$ & $\exp (-1 / T)$ \\
\hline
\end{tabular}


The leaky Schottky barrier model uses thermionic field emission as the $\sqrt{V}$ component for two reasons: Lee et al showed that this mechanism accurately modeled highresistivity GaAs (similar to high-resistivity CdTe) [40], and this current mechanism is controlled by the Schottky barrier height, as expected for these devices. Diffusion is also controlled by Schottky barrier height, but Kosyachenko et al indicated that this current is negligible [22]. Generation is not controlled by Schottky barrier height, so it is a less attractive model but may still be correct. Without more knowledge about the CdTe material, it could be difficult to determine the correct mechanism using the present current-voltage measurements; however, thermionic field emission, diffusion, and generation have different temperature dependencies, so current-voltage measurements over a wider range of temperatures may reveal which current mechanism better describes the $\sqrt{V}$ current component. With more temperature data or information about the CdTe material, the leaky Schottky model will be updated to consider dominant diffusion or generation current.

The linear dependence seen in the no sputter etch device can only be due to ohmic leakage. This supports the leaky Schottky model, where the inhomogeneous barrier was modeled as an ohmic leakage path in parallel with an ideal, homogenous barrier. Since the sputter etch removes the native oxide and decreases surface defect density, the Schottky barrier should be more homogeneous in this device. The analysis in figure 6.16 and the leaky Schottky model, section 5.2, both support this conclusion. The numerical models attempt to describe the devices using a homogeneous barrier. The resulting current-voltage characteristics clearly only have a $\sqrt{V}$ current component, so this model cannot accurately describe both devices in reverse bias. 
More complex modeling would increase our understanding of these devices. A detailed statistical analysis of barrier homogeneity would provide more information about the effect of the sputter etch on barrier formation. More complex numerical models are also required, since the leaky Schottky model only describes conduction in reverse bias. Numerical models that reproduce measured forward and reverse characteristics would provide valuable insight into the conduction mechanisms in this device. The more complex numerical model would incorporate an inhomogeneous barrier, surface states, a native oxide layer, and modified surface stoichiometry caused by the sputter etch. However, to improve these models we need more information about surface and bulk trap states. Characterizing the surface states of these devices would indicate whether or not the surface defect energy levels extracted from the leaky Schottky model are physically meaningful. Characterizing the bulk trap density would refine the leaky Schottky model and the numerical model; since thermionic field emission, diffusion, and generation currents are related to depletion width.

Capacitance versus voltage $(\mathrm{C}-\mathrm{V})$ measurements could yield information about defect density, but near-intrinsic materials are difficult to characterize with $\mathrm{C}-\mathrm{V}$, due to the wide reverse bias depletion region. Surface defects and trapped charge can also make $\mathrm{C}-\mathrm{V}$ measurements difficult to analyze. A more accurate value for the dark series resistance would improve $\mathrm{C}-\mathrm{V}$ modeling of these devices, since the actual device capacitance is related to the measured capacitance, conductance, and series resistance.

Illuminating the devices during current versus voltage measurements can yield more information about diode series resistance. Under uniform illumination, dark series resistance can be determined from an unknown light source using only current-voltage 
data. This information could help to refine I-V and C-V models. Obtaining the series resistance for the device under illumination (not the dark series resistance) may provide insight into the dominant trap energy level of photo-generated carriers; this could help refine the trap levels in the numerical model. 


\section{CONCLUSIONS AND FUTURE WORK}

This thesis investigates the current versus voltage characteristics of two unique Schottky devices: one with an argon ion sputter etch before Schottky contact deposition and one without. Initial simulations show that Schottky devices have lower reverse bias leakage than ohmic devices, and at $200 \mathrm{~V}$ reverse bias measurements show more than $50 \%$ lower leakage in the sputter etch device compared to the no sputter etch device.

\subsection{Analytical and Numerical Modeling}

Table 7.1 shows the barrier heights from the three different $500 \mu \mathrm{m}$ device models.

Table 7.1 Extracted Barrier Heights for Analytical and Numerical Models

\begin{tabular}{|c|c|c|c|}
\hline & Thermionic emission & Leaky Schottky barrier & Numerical \\
\hline No sputter etch & $0.71 \mathrm{eV}$ & $0.865 \mathrm{eV}$ & $0.781 \mathrm{eV}$ \\
\hline Sputter etch & $0.75 \mathrm{eV}$ & $0.884 \mathrm{eV}$ & $0.81 \mathrm{eV}$ \\
\hline $\begin{array}{c}\text { Improvement due to } \\
\text { sputter etch }\end{array}$ & $0.04 \mathrm{eV}$ & $0.019 \mathrm{eV}$ & $0.029 \mathrm{eV}$ \\
\hline
\end{tabular}

Although the models disagree on the Schottky barrier height, they all indicate that the sputter etch increases the effective barrier height by at least $0.019 \mathrm{eV}$. For models controlled by the barrier height, this small change corresponds to a $47 \%$ decrease in 
reverse bias current at room temperature; this decrease is very close to the $50 \%$ reduced current observed in measurements.

Initial analytical models show that these devices are not accurately described by thermionic emission, but the thermionic emission model suggests that the devices have an inhomogeneous barrier. In reverse bias, these devices are modeled by thermionic field emission with a low tunneling component and an inhomogeneous barrier, represented as an ohmic leakage path. The sputter etch device shows significantly less leakage through the barrier, suggesting that the argon ion sputter etch improves barrier homogeneity. Both models indicate a higher Schottky barrier in the sputter etch devices, suggesting again that the sputter etch improves contact formation.

The accuracy of numerical models is limited by the complexity of the model. The device model does not account for native oxide, barrier inhomogeneity, or interface states. However, the numerical modeling still shows that anode work function controls the reverse leakage current over a large range of trap configurations; as with the analytical models, these simulations also indicate that the sputter etch device has a higher anode Schottky barrier. Without an inhomogeneous barrier providing an ohmic current component, these models could not accurately reproduce the experimental data.

This thesis provides an accurate model for describing reverse bias conduction and shows that these devices must be modeled with a inhomogeneous Schottky barrier. Further measurements are required to resolve whether generation, diffusion, or thermionic field emission describe the $\sqrt{V}$ dependence seen in the reverse bias current; more knowledge of trap density will also help resolve this and will refine the analytical and numerical models. Understanding the current mechanisms in these devices will 
suggest ways to increase barrier height, contact homogeneity, and contact reproducibility.

These improved blocking contacts will reduce leakage current and should ultimately provide better energy resolution in these gamma ray detectors.

\subsection{Future Work}

Beyond the scope of this thesis, there are many other paths to reducing the reverse bias leakage current. Cadmium Zinc Telluride (CZT) is a promising detector material with higher resistivity, $\rho>10^{10} \Omega \cdot \mathrm{cm}[18]$. The higher resistivity typically yields lower leakage current, but this material has higher defect densities, making Schottky contact formation more difficult. Despite this complication, CZT is the primary material being researched, and many paths to improved barrier formation and lower leakage current are under investigation.

Although we attribute the improved barrier formation in the sputter etch devices to decreased surface defect density, the surface stoichiometry may play a role in determining the barrier height and homogeneity; previous reports indicate better Schottky contact formation on the B-face of (111) CdTe [13]. Fabricating contacts on the A- and B-faces with and without sputter etches will yield more information about the properties that effect contact formation. Surface conduction measurements and x-ray photon spectroscopy have already shown that the sputter etch makes the A-face stoichiometrically similar to the B-face.

Passivating the surface with sulfur compounds, such as ammonium sulfide and thiourea, has been shown to decrease defect density and leakage current [52], [53], [54]. Devices treated with ammonium sulfide have already been characterized with current 
versus voltage measurements and show promising results. Further analysis of surface properties is necessary to understand the effects of these treatments.

Depositing amorphous semiconductor layers underneath the Schottky contact is another method for decoupling contact formation and surface defects [55], [56]. Devices with amorphous germanium and amorphous silicon layers under the Schottky contact have already been fabricated and electrically characterized. These devices show increased barrier heights and decreased leakage. More measurements and numerical models are required to better understand these interfacial layers.

As detailed in section 6.3, the next step for the research in this thesis is to take more current-voltage measurements over a wider temperature range; this will help refine the leaky Schottky model. A more complex numerical model is also necessary to accurately describe these devices; this model must have an inhomogeneous barrier to account for the ohmic leakage primarily observed in the no sputter etch device. A better understanding of the CdTe material will also help to further refine the models in this thesis. Finally, all of these models will be adapted to CdZnTe and the other devices described in this section. 


\section{REFERENCES}

[1] Wikipedia contributors, "Scintillator," Wikipedia, The Free Encyclopedia, Oct. 5, 2009. [Online]. Available: http://en.wikipedia.org/wiki/Scintillator. [Accessed Nov. 7, 2009].

[2] Wikipedia contributors, “Gamma Spectroscopy,” Wikipedia, The Free Encyclopedia, Nov. 4, 2009. [Online]. Available: http://en.wikipedia.org/wiki/Gamma_spectroscopy. [Accessed Nov. 7, 2009].

[3] G. F. Knoll, Radiation Detection and Measurement, 3rd ed. New York: John Wiley, 2000.

[4] R. Nikolic, A. Conway, T. F. Wang, A. Nelson, and S. Payne, "CZT sandwich detector for gamma radiation,” Lawrence Livermore National Laboratory LDRD Proposal FY08.

[5] Wikipedia contributors, “Attenuation Coefficient," Wikipedia, The Free Encyclopedia, Nov. 4, 2009. [Online]. Available: http://en.wikipedia.org/wiki/Attenuation_coefficient. [Accessed Dec. 5, 2009].

[6] H. L. Malm, “A mercuric iodide gamma-ray spectrometer,” IEEE Transactions on Nuclear Science, vol. 19, no. 3, pp. 263-265, June 1972. [Online]. Available: ieeexplore.ieee.org. [Accessed Dec. 5, 2009].

[7] H. Spieler, "Introduction to Radiation Detectors and Electronics: 8.1 The Detector Signal," Spring 1998. [Online]. Available: http://www-physics.lbl.gov/ spieler/physics_198_notes/. [Accessed Dec. 5, 2009].

[8] K. Kim, S. Ahn, S. An, J. Hong, Y. Yi, and S. Kim, “Carrier transport properties in high resistivity polycrystalline CdZnTe material," Current Applied Physics, vol. 7, no. 3, pp. 296-299, March 2007. [Online]. Available: www.sciencedirect.com. [Accessed Dec. 7, 2009]. 
[9] T. Schulman, "Si, CdTe and CdZnTe radiation detectors for imaging applications," Ph.D. dissertation, University of Helsinki, Finland, 2006.

[10] C. R. Wie, "Crystal Viewer," 1997. [Online]. Available: http://jas.eng.buffalo.edu/education/solid/unitCell/home.html. [Accessed Dec. 7, 2009].

[11] “Microscopic Solid Structures." [Online]. Available: http://www.cartage.org.lb/en/themes/Sciences/Physics/SolidStatePhysics/AtomicBonding/CrystalStru cture/MicroscopicStructures/MicroscopicStructures.htm. [Accessed Dec. 7, 2009].

[12] T. Wichert, "II-VI Semiconductor," May 2005. [Online]. Available: http://www.nssp.unisaarland.de/forschung/halbleiterenglish.htm. [Accessed Dec. 7, 2009].

[13] T. Ozaki, Y. Iwase, H. Takamura, and M. Ohmori, "Thermal treatment of CdTe surfaces for radiation detectors," Nuclear Instruments and Methods in Physics Research Section A: Accelerators, Spectrometers, Detectors and Associated Equipment, vol. 380, no. 1-2, pp. 141-144, Oct. 1996. [Online]. Available: www.sciencedirect.com. [Accessed Dec. 8, 2009].

[14] K. Hermann, "Surface Explorer." [Online]. Available: http://w3.rzberlin.mpg.de/ rammer/surfexp_prod/SXinput.html. [Accessed Dec. 7, 2009].

[15] M. Fiederle, et al., "Modified compensation model of CdTe," Journal of Applied Physics, vol. 84, no. 12, pp. 6689-6692, Dec. 1998. [Online]. Available: scitation.aip.org. [Accessed Dec. 7, 2009].

[16] SILVACO International, ATLAS User's Manual, SILVACO International, 2007.

[17] D. Neamen, Semiconductor Physics and Devices, $3^{\text {rd }}$ ed. New York: McGraw-Hill, 2002.

[18] T. Takahashi and S. Watanabe, "Recent progress in CdTe and CdZnTe detectors," IEEE Transactions on Nuclear Science, vol. 48, no. 4, pp. 950-959, Aug. 2001. [Online]. Available: ieeexplore.ieee.org. [Accessed Dec. 7, 2009].

[19] M. Gloeckler, A. L. Fahrenbruch, and J. R. Sites, "Numerical modeling of CIGS and CdTe solar cells: setting the baseline," Proceedings of 3rd World Conference on Photovoltaic Energy Conversion, 2003, vol. 1, no. 1, pp. 491-494, May 2003. [Online]. Available: ieeexplore.ieee.org. [Accessed Dec. 7, 2009]. 
[20] H. Cordes and R. Schmid-Fetzer, "The role of interfacial reactions in Pt/CdTe contact formation," Semiconductor Science and Technology, vol. 9, no. 11, pp. 2085-2096, Nov. 1994. [Online]. Available: www.iop.org. [Accessed Dec. 7, 2009].

[21] K. Suzuki, S. Seto, T. Sawada, and K. Imai, "Carrier transport properties of HPB CdZnTe and THM CdTe:Cl," IEEE Transactions on Nuclear Science, vol. 49, no. 3-2, pp. 1287-1291, June 2002. [Online]. Available: ieeexplore.ieee.org. [Accessed Dec. 7, 2009].

[22] L. A. Kosyachenko, V. M. Sklyarchuk, O. F. Sklyarchuk, O. L. Maslyanchuk, V. A. Gnatyuk, and T. Aoki, "Higher voltage Ni/CdTe Schottky diodes with low leakage current," IEEE Transactions on Nuclear Science, vol. 56, no. 4, pp. 1827-1834, Aug. 2009. [Online]. Available: ieeexplore.iee.org. [Accessed Dec. 7, 2009].

[23] D. R. Lide and W. M. Haynes, CRC Handbook of Chemistry and Physics, $90^{\text {th }}$ ed. London, England: Taylor \& Francis Group, 2009. [E-book] Available: http://www.hbcpnetbase.com/

[24] E. Janik and R. Triboulet, "Ohmic contacts to p-type cadmium telluride and cadmium mercury telluride," Journal of Physics D: Applied Physics, vol. 16, no. 12, pp. 2333-2340, Dec. 1983. [Online]. Available: www.iop.org. [Accessed Dec. 7, 2009].

[25] Y. Iwase, M. Funaki, A. Onozuka, and M. Ohmori, “A 90 element CdTe array detector,” Nuclear Instruments and Methods in Physics Research Section A: Accelerators, Spectrometers, Detectors and Associated Equipment, vol. 322, no. 3, pp. 628-632, Nov. 1992. [Online]. Available: www.sciencedirect.com. [Accessed Dec. 7, 2009].

[26] M. J. Mescher, T. E. Schlesinger, J. E. Toney, B. A. Brunett, and R. B. James, "Development of dry processing techniques for CdZnTe surface passivation," Journal of Electronic Materials, vol. 28, no. 6, pp. 700-704, June 1999. [Online]. Available: www.springerlink.com. [Accessed Dec. 8, 2009].

[27] Wikipedia contributors, "Buffered oxide etch," Wikipedia, The Free Encyclopedia, May 29, 2009. [Online]. Available: http://en.wikipedia.org/wiki/Buffered_oxide_etch. [Accessed: Nov. 8, 2009].

[28] Wikipedia contributors, "Triaxial Cable," Wikipedia, The Free Encyclopedia, July 1, 2009. [Online]. Available: http://en.wikipedia.org/wiki/Triaxial_cable. [Accessed: Dec. 7, 2009]. 
[29] B. Verzi, "Common Mistakes of Parametric Test and how to avoid them!," Feb. 2005. [Online]. Available: http://ewh.ieee.org/r5/central_texas/eds/files/feb_meeting_common_mistakes.ppt. [Accessed: Dec. 7, 2009].

[30] W. S. Wang, C. Ho, and T. M. Chuang, "Investigation of PtSi/p-Si Schottky barrier height using I-VT technique” Materials Chemistry and Physics, vol. 52, no. 1, pp. 88-91, Oct. 1997. [Online]. Available: www.sciencedirect.com. [Accessed Dec. 7, 2009].

[31] Q. Li, W. Jie, L. Fu, and G. Zha, "Investigation on interface barrier of Au-CdZnTe contacts" Nuclear Instruments and Methods in Physics Research A, vol. 564, no. 1, pp. 544-548, Aug. 2006. [Online]. Available: www.sciencedirect.com. [Accessed Dec. 7, 2009].

[32] Z. Ahmad and M. H. Sayyad, "Extraction of electronic parameters of Schottky diode based on an organic semiconductor methyl-red," Physica E: Low-dimensional Systems and Nanostructures, vol. 41, no. 4, pp. 631-634, Feb. 2009. [Online]. Available: www.sciencedirect.com. [Accessed Dec. 8, 2009].

[33] D. Donoval, M. Barus, and M. Zdimal, “Analysis of I-V measurements on PtSi-Si Schottky structures in a wide temperature range," Solid-State Electronics, vol. 34, no. 12, pp. 1365-1373, Dec. 1991. [Online]. Available: www.sciencedirect.com. [Accessed Dec. 8, 2009].

[34] N. T. Tam and T. Chot, "Experimental Richardson constant of metal-semiconductor Schottky barrier contacts,” physica status solidi (a), vol. 93, no. 1, pp. K91-K95, Feb. 2006. [Online]. Available: www3.interscience.wiley.com. [Accessed Dec. 8, 2009].

[35] N. Tōyama, "Variation in the effective Richardson constant of a metal-silicon contact due to metalfilm thickness," Journal of Applied Physics, vol. 63, no. 8, pp. 2720-2724, April 1988. [Online]. Available: scitation.aip.org. [Accessed Dec. 8, 2009].

[36] D. K. Schroder, Semiconductor Material and Device Characterization, $2^{\text {nd }}$ ed. New York: John Wiley, 1998.

[37] R. T. Tung, "Recent advances in Schottky barrier concepts," Materials Science and Engineering: R: Reports, vol. 35, no. 1-3, pp. 1-138, Nov. 2001. [Online]. Available: www.sciencedirect.com. [Accessed Dec. 8, 2009]. 
[38] E. H. Rhoderick, Metal-Semiconductor Contacts, $2^{\text {nd }}$ ed. New York: Oxford University Press, 1988.

[39] L. A. Kosyachenko, O. L. Maslyanchuk, and V. M. Sklyarchuk, "Special features of charge transport in Schottky diodes based on semi-insulating CdTe," Semiconductors, vol. 39, no. 6, pp. 722-729, June 2005. [Online]. Available: www.springerlink.com. [Accessed Dec. 8, 2009].

[40] T. C. Lee, H. L. Au, T. P. Chen, C. C. Ling, S. Fung, and C. D. Beling, "Reverse I-V characteristics of Au/semi-insulating InP (100)," Semiconductor Science and Technology, vol. 8, no. 5, pp. 709-711, May 1993. [Online]. Available: www.iop.org. [Accessed Dec. 8, 2009].

[41] D. M. Hofmann, W. Stadler, P. Christmann, and B. K. Meyer, "Defects in CdTe and $\mathrm{Cd}_{1-\mathrm{x}} \mathrm{Zn}_{\mathrm{x}} \mathrm{Te}, "$ Nuclear Instruments and Methods in Physics Research Section A: Accelerators, Spectrometers, Detectors and Associated Equipment, vol. 380, no. 1-2, pp. 117-120, Oct. 1996. [Online]. Available: www.sciencedirect.com. [Accessed Dec. 8, 2009].

[42] M. Ayoub, et al., "Annealing effects on defect levels of $\mathrm{CdTe}: \mathrm{Cl}$ materials and the uniformity of the electrical properties," IEEE Transactions on Nuclear Science, vol. 50, no. 2, pp. 229-237, April 2003. [Online]. Available: ieeexplore.ieee.org. [Accessed Dec. 8, 2009].

[43] R. Soundararajan, K. G. Lynn, S. Awadallah, C. Szeles, S. H. Wei, "Study of defect levels in CdTe using thermoelectric effect spectroscopy," Journal of Electronic Materials, vol. 35, no. 6, pp. 13331340, June 2006. [Online]. Available: www.springerlink.com. [Accessed Dec. 8, 2009].

[44] A. Castaldini, A. Cavallini, B. Fraboni, P. Fernandez, and J. Piqueras, "Deep energy levels in CdTe and CdZnTe," Journal of Applied Physics, vol. 83, no. 4, pp. 2121-2126, Feb. 1998. [Online]. Available: scitation.aip.org. [Accessed Dec. 8, 2009].

[45] P. J. Sellin, A. W. Davies, A. Lohstroh, M. E. Öhzan, and J. Parkin, "Drift mobility and mobilitylifetime products in CdTe:Cl grown by the travelling heater method," IEEE Transactions on Nuclear Science, vol. 52, no. 6, pp. 3074-3078, Dec. 2005. [Online]. Available: ieeexplore.ieee.org. [Accessed Dec. 8, 2009].

[46] S. M. Sze, Physics of Semiconductor Devices, $2^{\text {nd }}$ ed. New York: John Wiley and Sons, 1981. 
[47] S. Gilliland, J. González, H. S. Güder, A. Segura, I. Mora, and V. Muñoz, "Pressure and temperature dependence of the band-gap in CdTe," Physica Status Solidi (b), vol. 235, no. 2, pp. 441-445, Jan. 2003. [Online]. Available: www3.interscience.wiley.com. [Accessed Dec. 8, 2009].

[48] S. Yamanaka, S. Tsushima, H. Shimizu, and Y. Tokumaru, “Temperature dependence of photocurrent and band gap of Cl-doped semi-insulating CdTe with evaporated Au electrodes," Japanese Journal of Applied Physics, vol. 41, no. 1, pp. 5538-5541, May 2002. [Online]. Available: jjap.ipap.jp. [Accessed Dec. 8, 2009].

[49] J. Fink, P. Lodomez, H. Krüger, H. Pernegger, P. Weilhammer, and N. Wermes, “TCT characterization of different semiconductor materials for particle detection," Nuclear Instruments and Methods in Physics Research Section A: Accelerators, Spectrometers, Detectors and Associated Equipment, vol. 565, no. 1, pp. 227-233, Sept. 2006. [Online]. Available: www.sciencedirect.com. [Accessed Dec. 8, 2009].

[50] J. S. Blakemore, "Semiconducting and other major properties of gallium arsenide," Journal of Applied Physics, vol. 53, no. 10, pp. R123- R181, Oct. 1982. [Online]. Available: scitation.aip.org. [Accessed Dec. 8, 2009].

[51] V. L. Dalal, A. B. Dreeben, and A. Triano, "Temperature dependence of hole velocity in p-GaAs," Journal of Applied Physics, vol. 42, no. 7, pp. 2864-2867, June 1971. [Online]. Available: scitation.aip.org. [Accessed Dec. 8, 2009].

[52] M. Yamazato, et al., "Improvement in $\gamma$-ray detection quality of Al/CdTe/Pt Schottky-type radiation detector by sulfur treatment,” Japanese Journal of Applied Physics, vol. 45, no. 1, pp. L1263-L1265, Nov. 2006. [Online]. Available: jjap.ipap.jp. [Accessed Dec. 8, 2009].

[53] Y. V. Klevkov, S. A. Kolosov, and A. F. Plotnikov, "Effect of passivation of the p-CdTe surface in $\left(\mathrm{NH}_{4}\right)_{2} \mathrm{~S}_{\mathrm{x}}$ on the current-voltage characteristics of contacts," Semiconductors, vol. 40, no. 9, pp. 10481051, Sept. 2006. [Online]. Available: www.springerlink.com. [Accessed Dec. 8, 2009].

[54] J. R. Waldrop, "Direct variation of metal-GaAs Schottky barrier height by the influence of interface S, Se, and Te," Applied Physics Letters, vol. 47, no. 12, pp. 1301-1303, Dec. 1985. [Online]. Available: scitation.aip.org 
[55] J. Ivanco, H. Kobayashi, J. Almeida, and G. Margaritondo, "Unpinning of the Au/GaAs interfacial Fermi level by means of ultrathin undoped silicon interlayer inclusion," Journal of Applied Physics, vol. 87, no. 2, pp. 795-800, Jan. 2000. [Online]. Available: scitation.aip.org. [Accessed Dec. 8, 2009].

[56] J. C. Costa, T. J. Miller, F. Williamson, and M. I. Nathan, "Unpinned GaAs Schottky barriers with an epitaxial silicon layer," Journal of Applied Physics, vol. 70, no. 4, pp. 2173-2184, Aug. 1991. [Online]. Available: scitation.aip.org. [Accessed Dec. 8, 2009]. 


\section{APPENDIX A: SILVACO ATLAS SIMULATION CODE}

This code runs once for each temperature.

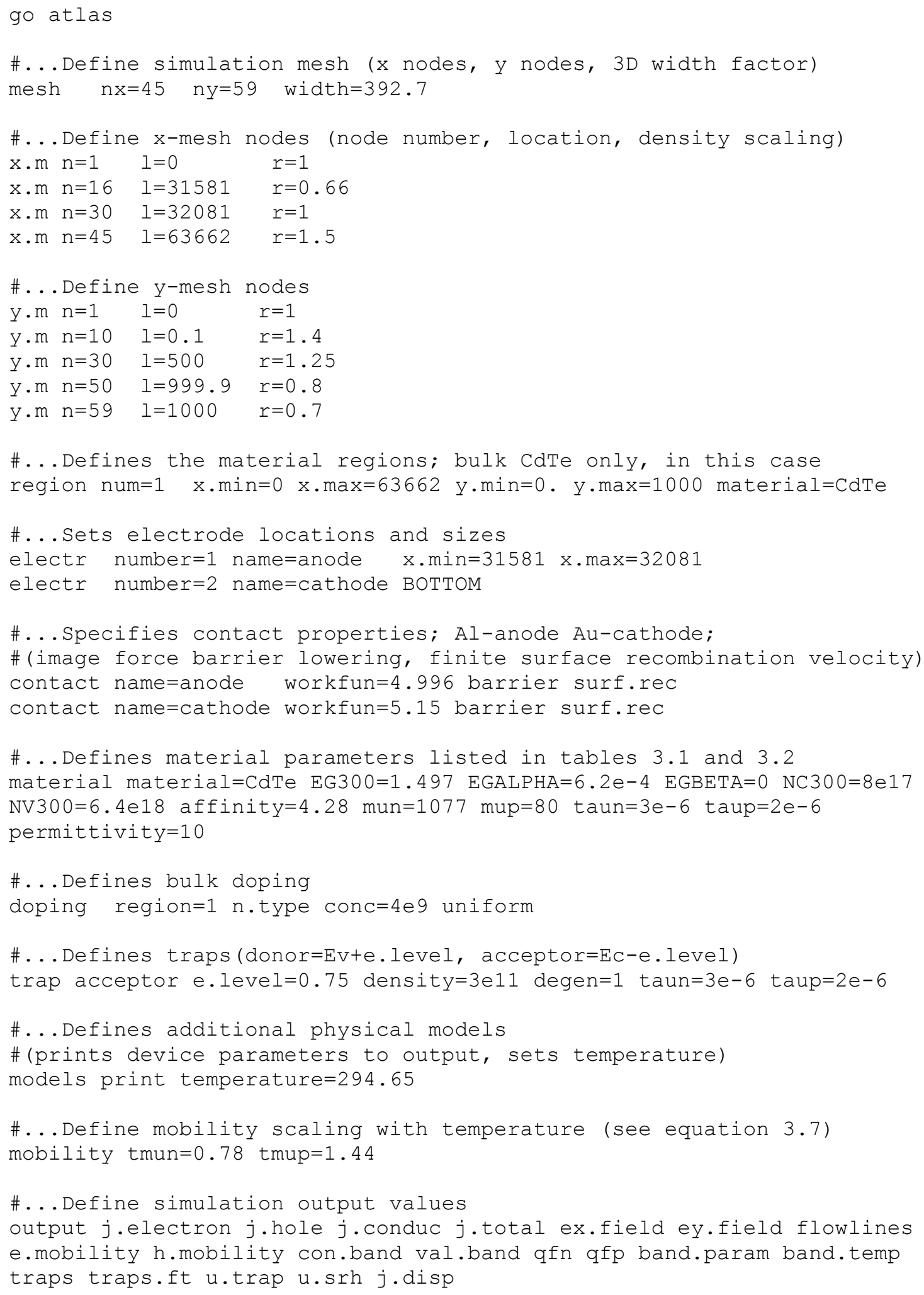




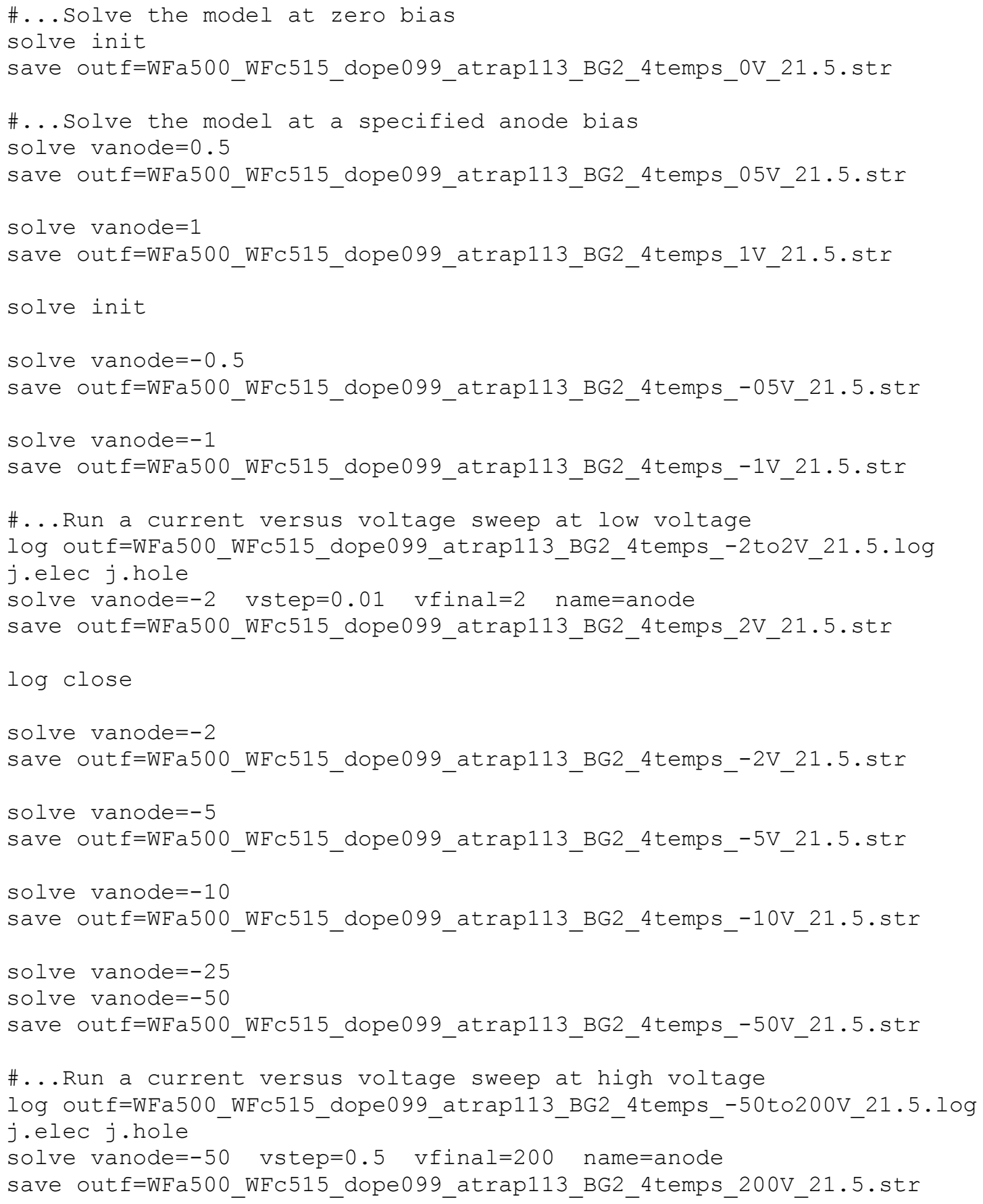

